Florida International University

FIU Digital Commons

FIU Electronic Theses and Dissertations

University Graduate School

3-30-2012

\title{
Identification, Isolation, and Characterization of Developmental Toxins from the Cyanobacterium Fischerella 52-1 Using the Zebrafish (Danio rerio) Embryo Model
}

Katherine E. Walton

Florida International University, kwalt001@fiu.edu

DOI: $10.25148 /$ etd.FI12050207

Follow this and additional works at: https://digitalcommons.fiu.edu/etd

\section{Recommended Citation}

Walton, Katherine E., "Identification, Isolation, and Characterization of Developmental Toxins from the Cyanobacterium Fischerella 52-1 Using the Zebrafish (Danio rerio) Embryo Model" (2012). FIU Electronic Theses and Dissertations. 645.

https://digitalcommons.fiu.edu/etd/645

This work is brought to you for free and open access by the University Graduate School at FIU Digital Commons. It has been accepted for inclusion in FIU Electronic Theses and Dissertations by an authorized administrator of FIU Digital Commons. For more information, please contact dcc@fiu.edu. 


\title{
FLORIDA INTERNATIONAL UNIVERSITY
}

Miami, Florida

\section{IDENTIFICATION, ISOLATION, AND CHARACTERIZATION OF \\ DEVELOPMENTAL TOXINS FROM THE CYANOBACTERIUM FISCHERELLA 52- \\ 1 USING THE ZEBRAFISH (DANIO RERIO) EMBRYO MODEL}

\author{
A thesis submitted in partial fulfillment of \\ the requirements for the degree of \\ MASTER OF SCIENCE \\ in \\ CHEMISTRY \\ by
}

Katherine Walton 
To: Dean Kenneth G. Furton

College of Arts and Sciences

This thesis, written by Katherine Walton, and entitled Identification, Isolation, and Characterization of Developmental Toxins from the Cyanobacterium Fischerella 52-1 Using the Zebrafish (Danio rerio) Embryo Model, having been approved in respect to style and intellectual content, is referred to you for judgment.

We have read this thesis and recommend that it be approved.

Kevin O'Shea

Kathleen Rein

John Berry, Major Professor

Date of Defense: March 30, 2012

The thesis of Katherine Walton is approved.

Dean Kenneth G. Furton College of Arts and Sciences

Dean Lakshmi N. Reddi University Graduate School

Florida International University, 2012 


\section{DEDICATION}

I dedicate this thesis to my family. Without their constant support and love I would not have made it this far. 


\section{ACKNOWLEDGMENTS}

I wish to thank my lab-mates, Asha Jaja and Gerald Berry, for their friendship and support. I also wish to thank Dr. Pat Gibbs for providing the needed zebrafish eggs and advice on zebrafish husbandry. I wish to thank Cesar Ramirez for patiently answering my numerous questions about the mass spectrometer and chromatography. I also wish to thank Dr. Miroslav Gantar for culturing the Fischerella 52-1 on multiple occasions. I wish to thank Dr. Rein's students, especially Ryan Cassell, Wentian Wang, and Li Liu for all of the assistance with the NMR. I also wish to thank Mayra Exposito for her assistance with the UV-vis and IR. Finally I would like to thank my committee members for their advice, patience, and understanding.

It has been with the help of these individuals that I was able to conduct my research. 


\author{
ABSTRACT OF THE THESIS \\ IDENTIFICATION, ISOLATION, AND CHARACTERIZATION OF \\ DEVELOPMENTAL TOXINS FROM THE CYANOBACTERIUM FISCHERELLA 52- \\ 1 USING THE ZEBRAFISH (DANIO RERIO) EMBRYO MODEL
}

by

Katherine Walton

Florida International University, 2012

Miami, Florida

Professor John Berry, Major Professor

Cyanobacteria, also known as blue-green algae, are known to produce a number of biologically active compounds. Extracts of cultured cyanobacteria isolated from South Florida sources were screened for possible developmental toxins using the zebrafish (Danio rerio) embryo as a model of vertebrate development. A strain of cyanobacteria, Fischerella 52-1, isolated from the Florida Everglades, was found to produce metabolites that caused a consistent developmental dysfunction in embryos exposed to lipophilic extract. Initial chemical characterization of the bioactive fraction identified a series of eight apparent indole-containing compounds. The two main components were purified using the zebrafish embryo model to guide the fractionation. Chemical characterization using 1- and 2-dimensional NMR, HESIMS, HRHESIMS, and IR determined that the two main compounds were the previously identified 12-epi-Hapalindole H Isonitrile, and a novel compound 12-epi-Ambiugine B Nitrile. The major contributor of the developmental defects detected in the zebrafish embryos was 12-epi-Hapalindole $\mathrm{H}$ Isonitrile. 


\section{TABLE OF CONTENTS}

CHAPTER

PAGE

1. SECONDARY METABOLITES OF THE CYANOBACTERIAL FAMILY

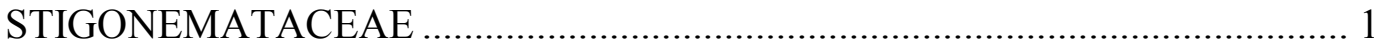

1.1 Secondary Metabolites of Cyanobacteria ................................................... 1

1.2 Secondary Metabolites from Stigonometaceae.............................................

1.3 Indole Alkaloids from Stigonemataceae ................................................ 9

1.3.1 Hapalindoles ................................................................... 12

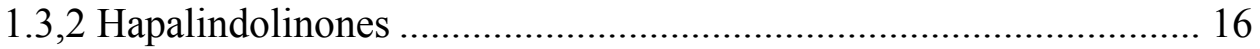

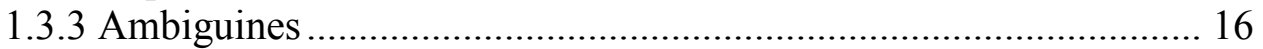

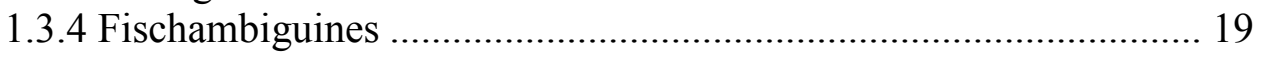

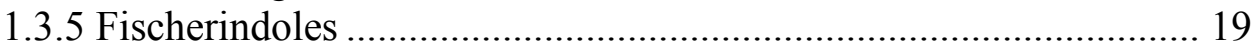

1.3.6 Welwitindolinones ................................................................. 20

1.4 Biosynthesis of Indole Alkaloids from Stigonemataceae ............................ 22

1.5 Structure-Activity Relationship of Indole Alkaloids from Stigonemataceae 29

2. TOXICOLOGICAL AND CHEMICAL INVESTIGATION OF FISCHERELLA

52-1 USING THE ZEBRAFISH (DANIO RERIO) EMBRYO MODEL............. 33

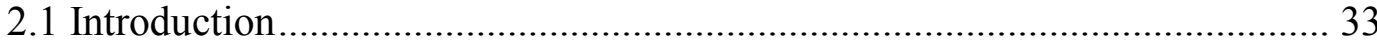

2.2 Zebrafish (Danio rerio) as a Toxicological Model...................................... 33

2.3 Use of the Zebrafish Embryo Model to Investigate Bioactive Metabolites from

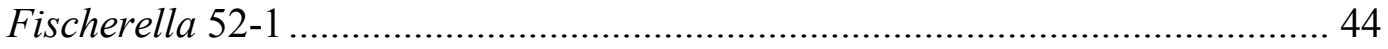

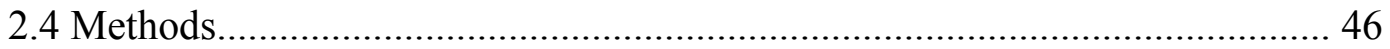

2.4.1 Fischerella 52-1 Culture ......................................................... 46

2.4.2 Extraction.............................................................................. 46

2.4.3 Optimized Extraction of ECS ..................................................... 47

2.4.4 HPLC Comparison of Bioactive Components of Fischerella 52-1

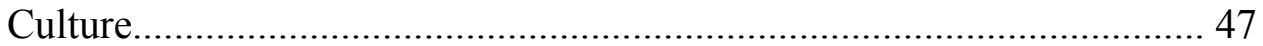

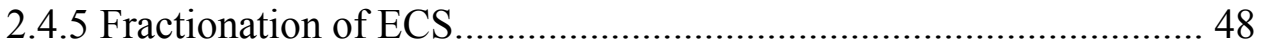

2.4.6 Zebrafish Embryo Bioassay ..................................................... 49

2.4.7 Embryo Removal Experiments .............................................. 51

2.4.8 Mosquito Larvicidal Assay ....................................................... 51

2.4.9 LC-MS of Indoles from ECS ................................................... 52

2.4.10 Toxin Solubility Analysis ...................................................... 53

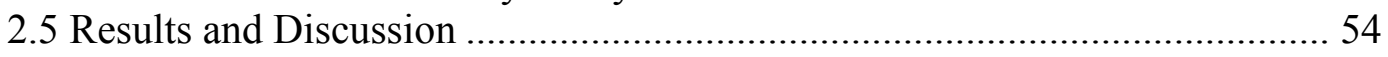

2.5.1 Toxicological Investigation of Crude Extracts ........................... 54

2.5.2 Isolation of Indole Alkaloids ................................................... 60

2.5.3 Chemical and Toxicological Investigation of Main Indole

Alkaloids ................................................................................. 62

2.5.4 Metabolite and Solubility Analysis............................................ 74

2.5.5 Mosquito Larvicidal Bioassay .............................................. 75

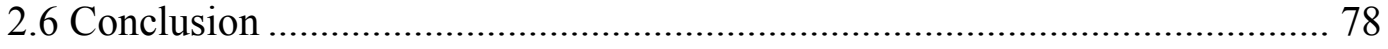


3. CHEMCAL CHARACTERIZATION OF MAJOR INDOLE ALKALOIDS

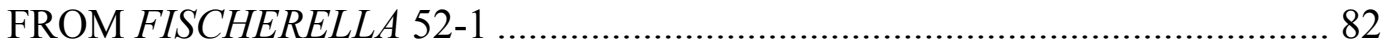

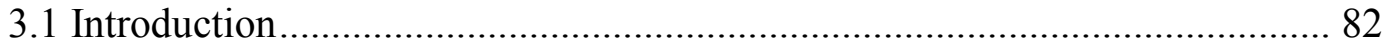

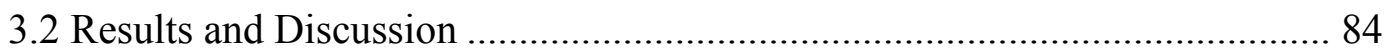

3.2.1 12-epi-Hapalindole H Isonitrile ............................................... 84

3.2.2 12-epi-Ambiguine B Nitrile.................................................. 106

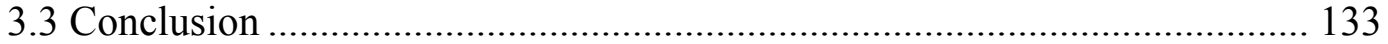

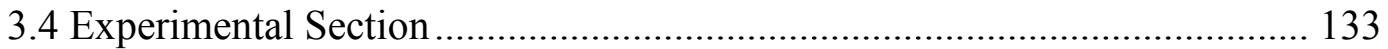

3.4.1 Purification............................................................................ 133

3.4.2 Ultraviolet and Infrared Analysis........................................... 134

3.4.3 Mass Spectrometer Analysis .................................................... 134

3.4.4 Nuclear Magnetic Resonance Spectroscopic Analysis ................. 135

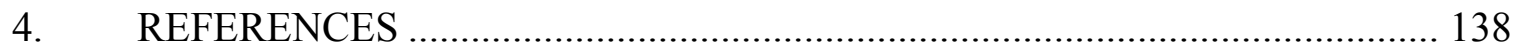




\section{LIST OF TABLES}

TABLE

PAGE

1. Toxin Classification (Codd et al., 1999 and Sivonen et al., 1996)........................ 2

2. Distribution of Indole Alkaloids in the Family Stigonemataceae........................ 10

3. Results of Zebrafish Embryo Bioassay on Crude Material ................................. 56

4. Composition of HPLC Fractions and Observed Bioactivity............................... 63

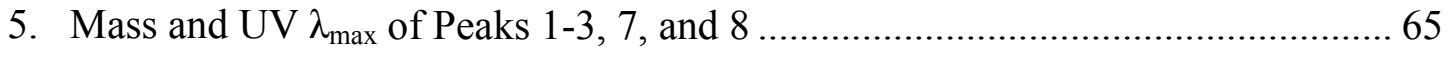

6. Mass Spectrometric Analysis of Peak 7 and 8 Solubility .................................. 74

7. ${ }^{13} \mathrm{C}$ NMR Chemical Shifts of 1 , Hapalindole H Isonitrile, 12-epi-Hapalindole H Isonitrile, and 12-epi-Hapalindole J Isonitrile ................................................ 94

8. ${ }^{1} \mathrm{H}$ NMR Comparison of 1 and Four Previously Identified Hapalindoles (400

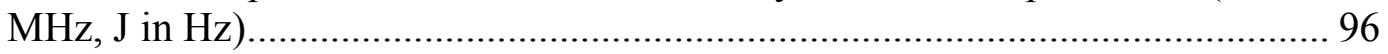

9. Proton Chemical Shifts from ${ }^{1} \mathrm{H}$ NMR of 2 and Ambiguine B Isonitrile (400

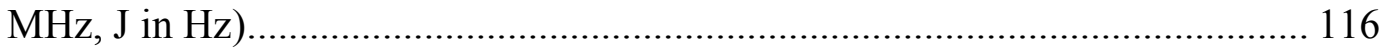

10. Carbon Chemical Shifts from ${ }^{13} \mathrm{C}$ NMR of 2 and Ambiguine B Isonitrile......... 118 


\section{LIST OF FIGURES}

FIGURE

PAGE

1. Secondary Metabolites from Family Stigonemataceae (Wright et al., 2005; Moore et al., 1987; Hagmann et al., 1996; Stratmann et al., 1994; Prinsep et al., 1992).. 5

2. Indole Alkaloid from Stigonemataceae (Stratmann et al., 1994; Smitka et al., 1992; Mo et al., 2010; Papke et al., 1997)....................................................... 11

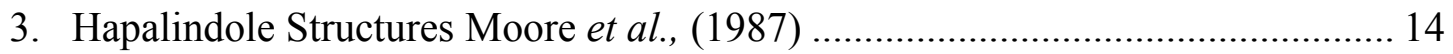

4. Structural Comparison of Fischerindole L and Hapalindole L. (Park et al., 1992)

5. Biosynthesis of 12-epi-Hapalindole E Isonitrile (Richter et al., 2008) 23

6. Biosynthesis of Hapalindoles, Fischerindoles, and Welwitindolinones (Richter et al., 2008)

7. Normal Zebrafish development; a) Day 1, b) Day 2, c) Day 3, d) Day 4, and e) Day 5 .....

8. Fish Embryo Bioassay Observations 5 dpf; a) Control, b) Biomass $250 \mu \mathrm{g} / \mathrm{mL}, \mathrm{c}$ ) Culture Medium $250 \mu \mathrm{g} / \mathrm{mL}$, d) ECS $10 \mu \mathrm{g} / \mathrm{mL}$ 57

9. HPLC analysi of different components of Fischerella 52-1; a) ECS, b) Culture Medium, and c) Biomass

10. Indole Chromaphore from ECS produced by Fischerella 52-1 obtained from the HPLC

11. HPLC Method 2 Chromatogram of ECS material

12. Bioactivity of Active Fractions Observed 5 dpf; a) Fraction 1, b) Fraction 3, c) Fraction 5, and d) Fraction 6

13. Zebrafish embryo bioassay of Peaks $7 \& 83$ dpf Observations. a) control, b) Peak $7,10 \mu \mathrm{g} / \mathrm{mL}$, c) Peak $8,5 \mu \mathrm{g} / \mathrm{mL}$, and d) $10 \mu \mathrm{g} / \mathrm{mL}$.

14. Observations 5 dpf of Zebrafish Embryo Bioassay of Peak 7; a) control, b) Peak 7 $10 \mu \mathrm{g} / \mathrm{mL}$

15. Zebrafish Embryo Assay Peak 8: 1) $5 \mu \mathrm{g} / \mathrm{mL}$ and 2) $10 \mu \mathrm{g} / \mathrm{mL} 5$ dpf Observations; a) Overall lack of pigment, b) abnormal curvature of the body, c) pericardial edema, d) control 
16. Zebrafish Embryo assay $5 \mu \mathrm{g} / \mathrm{mL}$ Peak 8 Observations; a) $2 \mathrm{dpf}$, b) $3 \mathrm{dpf}$, c) 5 dpf, d) Removed 2 dpf Observed 5 dpf, e) Removed 3 dpf Observed 5 dpf ........ 70

17. Zebrafish Embryo assay $10 \mu \mathrm{g} / \mathrm{mL}$ Peak 8 Observations; a) 2 dpf, b) 3 dpf, c) 5 dpf, d) Removed 2 dpf Observed 5 dpf, e) Removed 3 dpf Observed 5 dpf ........ 72

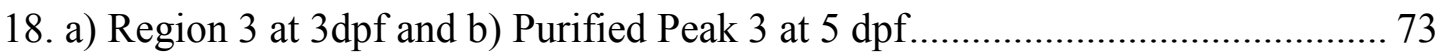

19. Development of Mosquito Lavae, images are not set to proper size ratio; a) $1^{\text {st }}$ Instar, b) $2^{\text {nd }}$ Instar, c) $3^{\text {rd }}$ Instar, and d) $4^{\text {th }}$ Instar. (Pictures provided by Gerald Berry)

20. Mosquito Larvacidal Assay Peak 8, a) Peak 8 Day 6 and b) Control 2nd Instar(Pictures produced by Gerald Berry) ........................................................ 77

21. Peak 3 and 7 identified in the Culture Medium by LCMS .................................. 80

22. Peak 3 and 7 identified in the Biomass Extract by LCMS .................................... 81

23. Structures of 12-epi-Hapalindole H Isonitrile (1) and 12-epi-Ambiguine B Nitrile (2) (Klein et al., 1995 and Smitka et al., 1992) ................................................ 82

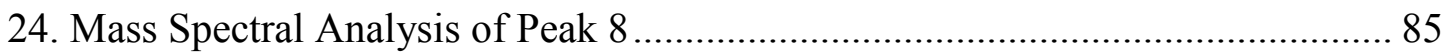

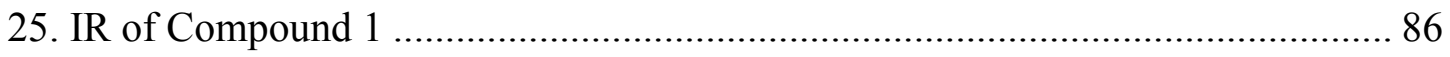

26. ${ }^{1} \mathrm{H}$ NMR in $\mathrm{D}_{2} \mathrm{O}$ and $\mathrm{MeOD}(*$ Denotes chemical shifts not assigned) The ethanol assigned above was used to clean the NMR tubes.................................................. 89

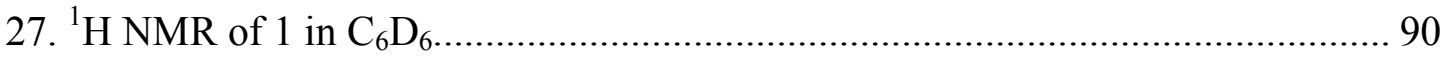

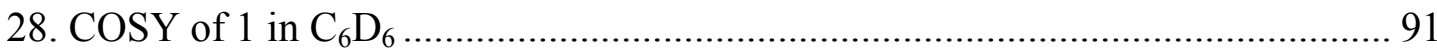

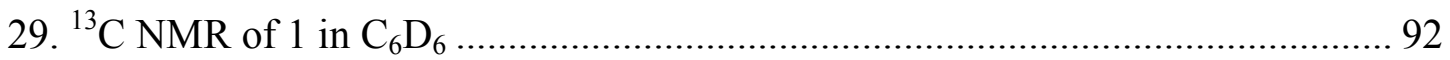

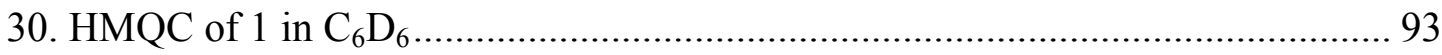

31. 12-epi-Hapalindole H Isonitrile Structure (Moore et al., 1987) ........................... 95

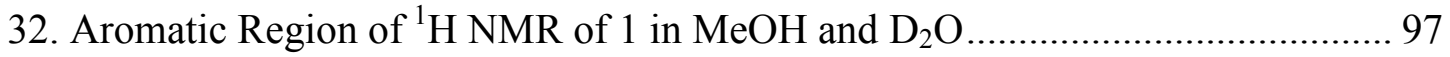

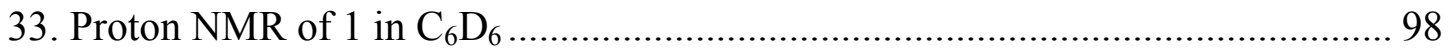

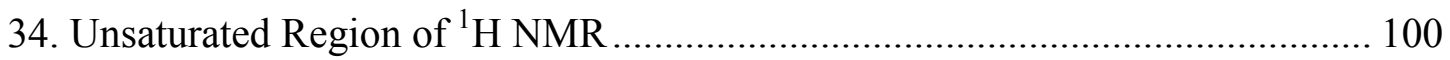

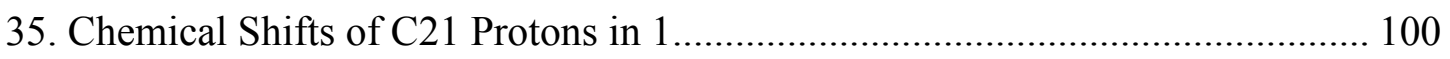


36. ${ }^{1} \mathrm{H}$ NMR of $\mathrm{C} 10$ and $\mathrm{C} 11$ Protons in 1 101

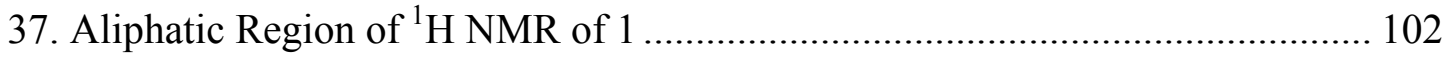

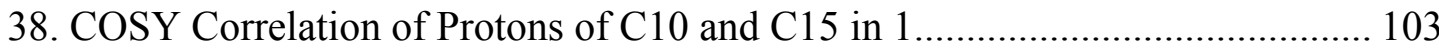

39. Assignment of Hydrogens using the HMQC of the Aliphatic Region of 1 ........ 105

40. Selection of 2 from the OT Velos HRHESIMS Data ...................................... 107

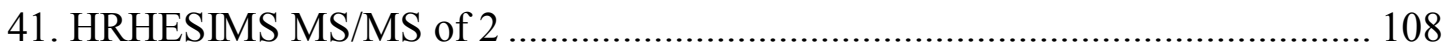

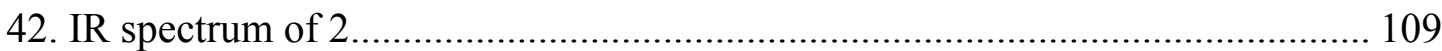

43. ${ }^{1} \mathrm{H}$ NMR of Compound 2 in $\mathrm{C}_{6} \mathrm{D}_{6}$ (* Denotes chemical shifts produced by impurities.)

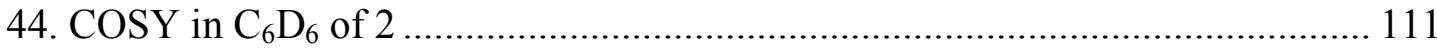

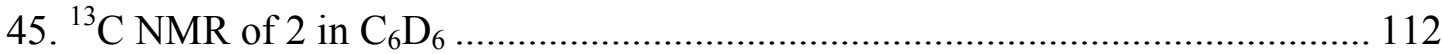

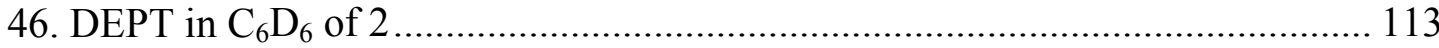

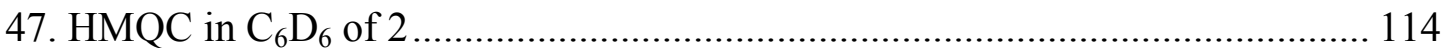

48. NOESY in $\mathrm{C}_{6} \mathrm{D}_{6}$ of 2. (*Denotes unassigned correlation.)............................. 115

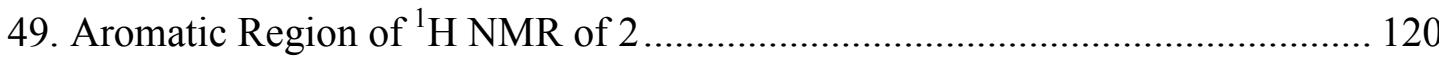

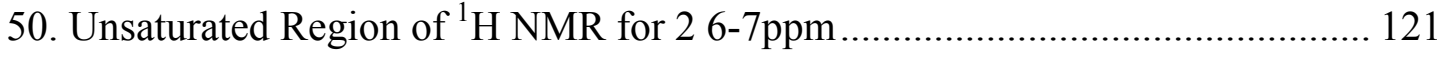

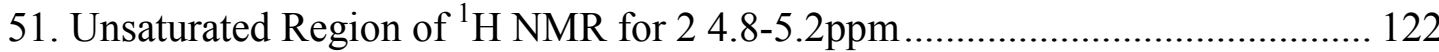

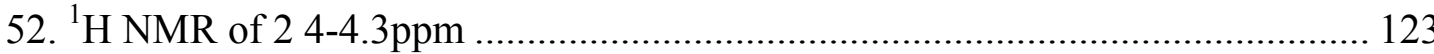

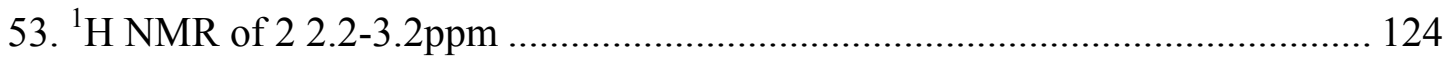

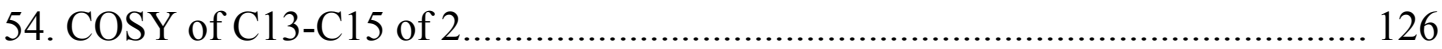

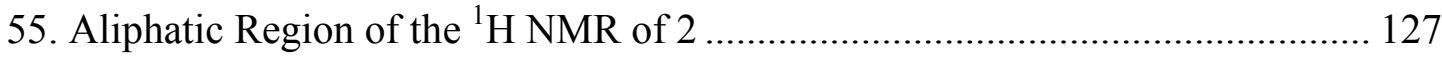

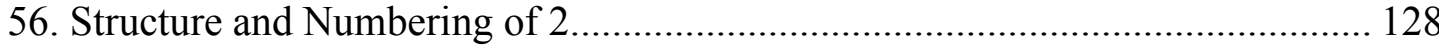




\section{SECONDARY METABOLITES OF THE CYANOBACTERIAL FAMILY STIGONEMATACEAE}

\subsection{Secondary Metabolites of Cyanobacteria}

Cyanobacteria, also called blue-green algae, are known to produce an array of bioactive secondary metabolites (Codd et al., 2005). Many of the bioactive secondary metabolites can be defined as toxins or as allelochemicals depending on the effect of the metabolite on other organisms (Leflaive et al., 2007). An allelochemical is defined as a compound that acts as a chemical signal that can either provide a detrimental or beneficial effect on neighboring organisms (Leao et al., 2010). A toxin, however, is defined as a compound that produces a negative health effect mostly observed towards humans and livestock (Leflaive et al., 2007). The classifications of toxins and allelochemicals are not two separate entities, and can possibly overlap to include the same compounds.

The cyanobacteria that produce these toxins have been found in a variety of environments which include freshwater, brackish water, marine, and terrestrial systems (Sivonen et al., 1996). Many of the cyanobacterial toxins were proposed to be linked to health issues which included poisoning of livestock and humans (Codd et al., 1999). Known toxin producing genera of cyanobacteria include Anabaena, Oscillatoria, Microcystis, Phormidium, Cylindrospermopsin, Aphanizomenon, Lyngbya, Nostoc, Anabaenopsis, Hapalosiphon, Fischerella, Nodulaia, Umezakia, and Schizothrix (Codd et al., 1999). The toxins isolated from these cyanobacteria can be classified by the biological systems affected. Many of these toxins are thus classified as neurotoxins, 
hepatotoxins, or dermatotoxins (Codd et al., 2005). Table 1 lists some of the better documented toxins under the designated classification.

Table 1: Toxin Classification (Codd et al., 1999 and Sivonen et al., 1996)

\begin{tabular}{|l|l|}
\hline Toxin Classification & Cyanobacterial Toxins \\
\hline Neurotoxins & Anatoxin-a, Anatoxin-a(s), Saxitoxin \\
\hline Hepatotoxins & Microcystin, Nodularin, Cylindrospemopsin \\
\hline Dermatotoxins & Lyngbyatoxin-a, aplysiatoxin, debromoaplysiatoxin \\
\hline
\end{tabular}

Many genera that have been shown to produce toxins were also observed to produce allelochemicals. Different cyanobacterial genera, that were determined to produce possible allelopathic compounds include Anabaena, Calothrix, Gomphosphaeria, Aphanizomenon, Hapalosiphon, Fischerella, Microcystis, Nodularia, Nostoc, Oscillatoria, Phormidium, Sytonema, and Trichormus (Gross 2003). The purpose of these compounds is not well known, but a possible reason for producing bioactive secondary metabolites could be to deter other organisms that would compete for limited resources, and, or that were possible predators (Berry et al., 2008). The previously proposed hypothesis would coincide with the observed bioactivity of many of these compounds which include the inhibition of insects, bacteria, fungi, and other algae (Leflaive et al., 2007). Cocultivation of cyanobacterial species with other algae has shown that some strains inhibit the growth of other algae; this inhibition has been shown to be true for Fischerella, and Nostoc (Gantar et al., 2008). The effectiveness of allelopathic compounds to inhibit algae and predators, especially insects, has some researchers suggesting using these compounds as insecticides and algicides (Berry et al., 2008). Since the allelopathic compounds already occur in nature it is possible that the 
bioactive metabolites pose as a more ecofriendly approach to remove unwanted species. Secondary metabolites from cyanobacteria stand as an excellent source of biologically active compounds. This chapter will investigate secondary metabolites including indole alkaloids produced by the cyanobacterial family Stigonemataceae.

\subsection{Secondary Metabolites from Stigonemataceae}

The family Stigonemataceae is classified as "true branching" filamentous cyanobacteria, and has been shown to produce a variety of bioactive compounds (Gugger et al., 2004). The main genera of the family that have been investigated are, Hapalosiphon, Fischerella, Westiella, Westiellopsis, and Stigonema (Stratmann et al., 1994; Prinsep et al., 1992; Ogino et al., 1996; Rao et al., 1999). Originally these genera occurred in the family Stigonemataceae, but have been subsequently reclassified under different families (Bisby et al., 2010).

Traditionally the organization of cyanobacteria was done by observing morphological and physiological details, but more contemporary methods utilize $16 \mathrm{~S}$ rRNA analysis which has led to a new classification system (Gugger et al., 2004). Given that much of the literature published utilized a classification system where all of the genera specified are classified under the same family, this paper will continue to use this system.

The majority of the secondary metabolites from the family Stigonemataceae include various alkaloids, polychlorinated aromatic compounds, and cyclic peptides (Stratmann et al., 1994; Falch et al., 1993; Moore et al., 1987). Secondary metabolites classified as toxins, like anatoxin-a, microcystin, cylindrospermopsin, and lyngbyatoxin are not well linked to this family. However, there was a documented instance of a 
freshwater Fischerella producing Microcystin LR in Brazil, and another case found Microcystin LA in Hapalosiphon hibernicus in Hawaii (Fiore et al., 2009; Prinsep et al., 1992). Most of the isolated bioactive metabolites from the family Stigonemataceae have been linked to antialgal, antimicrobial, and antifungal properties. Examples of these compounds are illustrated in Figure 1. 


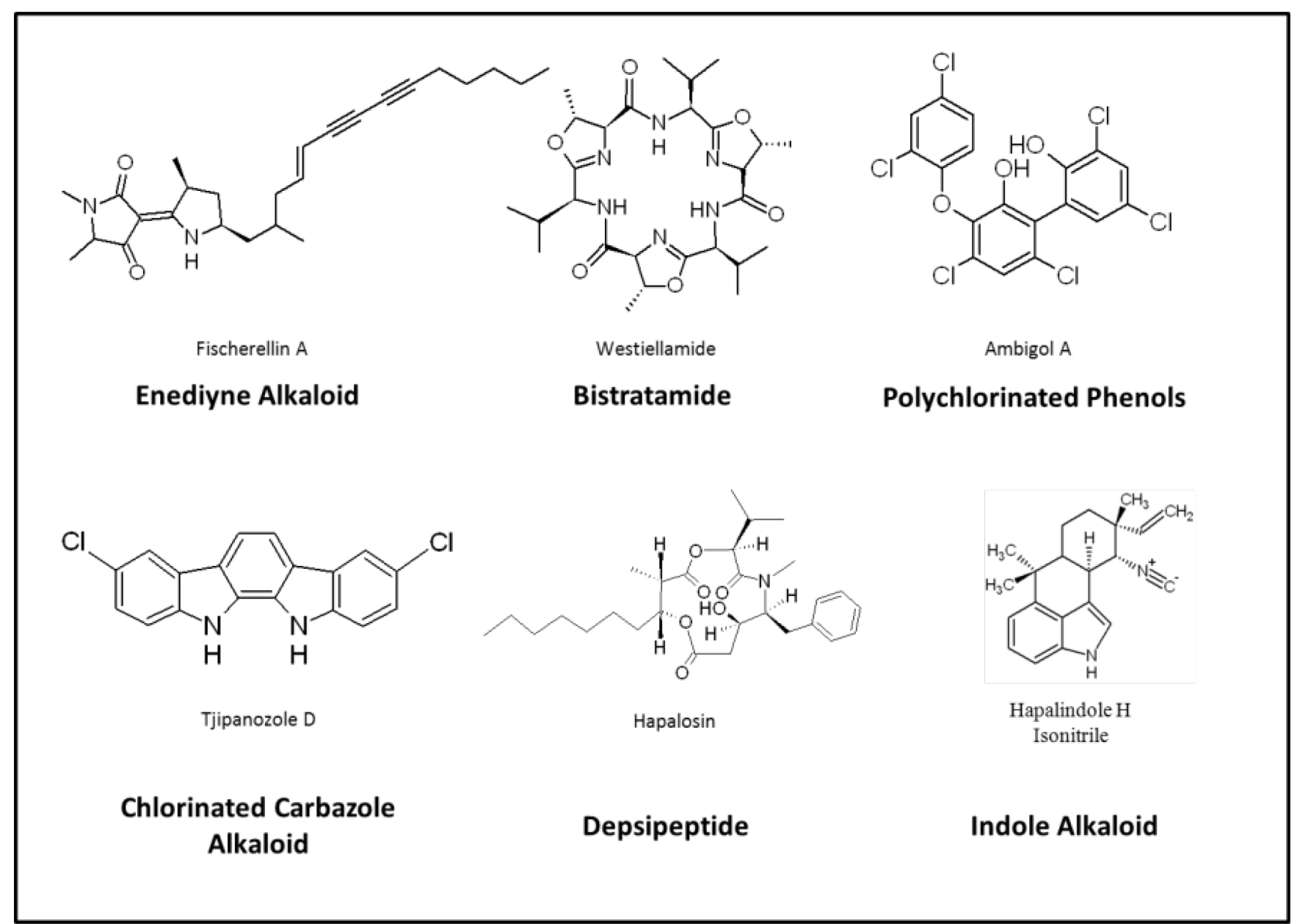

Figure 1: Secondary Metabolites from Family Stigonemataceae (Wright et al., 2005; Moore et al., 1987; Hagmann et al., 1996; Stratmann et al., 1994; Prinsep et al., 1992) 
Multiple polychlorinated compounds have been isolated from Fischerella ambigua (Falch et al., 1993; Wright et al., 2005). These compounds include the polyphenols Ambigol A, B, and C, 2,4-Dichlorobenzoic acid, and the polychlorinated carbazole alkaloid Tjipanazole D. Examples of these polychlorinated compounds are shown in Figure 1. Ambigols A and B and Tjipanazole D were isolated from Fischerella ambigua by Falch et al., (1993). Ambigol A was shown to inhibit cyclooxygenase, an enzyme responsible for inflammatory responses, inhibit HIV-1 reverse transcriptase, and have strong molluscicidal and antibacterial activity (Falch et al., 2003; Tan et al., 1991). Ambigol B was only moderately antimicrobial, molluscicidal, and cytotoxic. In the process of investigating metabolites produced by Fischerella ambigua under different culture conditions two new polychlorinated compounds, Ambigol $\mathrm{C}$ and 2,4Dichlorobenzoic acid, were isolated (Wright et al., 2005). In the Fischerella culture it was observed that Ambigol A and C, Tjipanazole D, and 2,4-Dichlorobenzoic acid were isolated but not Ambigol B. It was speculated that the observance of Ambigol C occurred at the expense of Ambigol B. Ambigol C was shown to be slightly bioactive against Plasmodium which is the organism responsible for malaria, and Trypanosoma which is responsible for Chagas disease (Wright et al., 2005; Valente et al., 2009). In the Wright et al., (2005) study the medium was also altered to try to replace the chlorine atoms with other halides like bromine and iodine, but this was unsuccessful. It was suggested that since the chlorines could not be altered that an enzyme must exist that was specific for chlorine halogenation. The occurrence of this type of enzyme in these cyanobacteria would explain why many of these secondary metabolites are chlorinated or coexist with non-chlorinated analogues. The compound 2,4-Dichlorobenzoic acid 
showed no apparent bioactivity against bacteria, fungi, molluscs, plasmodium, or trypanosome. The chlorinated carbazole alkaloid Tjipanazole D was isolated under both conditions and was shown to have moderate antibacterial bioactivity (Falch et al., 1993; Wright et al., 2005).

Other cyclic peptides besides microcystin have been isolated from members of Stigonemataceae family which include molecules that have either a depsipeptide or bistratamide moiety (Prinsep et al., 1992; Stratmann et al., 1994). Depsipeptides are peptides that have at least one of the amide nitrogens replaced with an oxygen to give and ester instead of an amide (Van Wagoner et al., 2007). The depsipeptide Hapalosin was isolated from Hapalosiphon welwitschii and was shown to have P-glycoprotein-mediated multidrug resistance (MDR) reversing activity (Stratmann et al., 1994). The activation of P-glycoprotein has been linked to MDR by acting as a drug efflux pump, and it was proposed that Hapalosin inhibited the pump thereby allowing accumulation of the drug in the cells (Stratmann et al., 1994). The proposed inhibitory capability of Hapalosin was further reinforced by the ability of the compound to increase the accumulation of the drugs taxol and vinblastine in P-glycoprotein-overexpressing, vinblastine-resistant subline (SKVLB1) of a human ovarian adenocarcinoma cell line. Further testing against other P-glycoprotein-overexpressing cells included human breast carcinoma showed that Hapalosin reversed the MDR to the point that vinblastine showed the same cytotoxicity towards the P-glycoprotein-overexpressing cells when combined with Hapalosin as with the non-overexpressing cells. Compared to Verapamil, an MDR reversing compound clinically studied, Hapalosin was shown to be more effective at reversing MDR. Depsipeptides similar to Hapalosin have been isolated from Microcystis, which might 
indicate a similar biosynthesis of peptide-based compounds by Microcystis and genera from Stigonemataceae (Stratmann et al., 1994).

The bistratamide type peptides include Westiellamide isolated from Westiellopsis and the Dendroamides isolated from Stigonema (Prinsep et al., 1992; Ogino et al., 1996). The cyclic moiety of the Dendroamides and Westiellamide have a similar structure to the natural product Bistratamide C previously isolated from a marine worm Lissoclinum bistratum.(Foster et al., 1992 and Prinsep et al., 1992) Dendroamides A-C were isolated from Stigonema dendroideum. As with Hapalosin, Dendroamide A was also shown to reverse MDR by inhibiting P-glycoprotein efflux pump and be more effective than Verapamil (Ogino et al., 1996). Dendroamides B and C were not shown to produce any reversal of MDR, which might be as a result of the sulfide side chain on Dendroamide B and sulfoxide side chain of Dendroamide C. Westiellamide, shown in Figure 1, was isolated from Westiellopsis prolifica and reported to be cyctotoxic towards KB and LoVo cell lines at a concentration of $2 \mu \mathrm{g} / \mathrm{mL}$ (Prinsep et al., 1992). Whether this compound reverses MDR is not specified in the isolation study, but the structural similarity of Westiellamide and Dendroamide A might suggest that Westiellamide could also reverse MDR.

A number of alkaloids have been isolated from Fischerella, Hapalosiphon, Westiella, and Westiellopsis that include indole and enediyne containg alkaloids, which are also depicted in Figure 1 (Moore et al., 1987; Stratmann et al., 1994; Smitka et al., 1992; Hagmann et al., 1996). The main enediyne, unsaturated polyacetylene, containing alkaloids are Fischerellin A and B, isolated from Fischerella muscicola by Hagmann et al., (1996). Fischerellin A was shown to produce a wide range of bioactivities which 
include anticyanobacterial, antialgal, fungicidal, toxicity towards rotifer and crustacean, and inhibition of photosystem II which produces an herbicidal activity. A moderately algicidal enediyne containing compound Fischerellin B was also isolated from Fischerella muscicola by Papke et al., (1997). Out of the alkaloids isolated from Stigonemataceae, the indole alkaloids are an important and widespread group of compounds that are addressed in the following section.

\subsection{Indole Alkaloids from Stigonemataceae}

The genera that have so far been shown to produce these indole alkaloids are Fischerella, Hapalosiphon, Westiella, and Westiellopsis (Moore et al., 1987; Stratmann et al., 1994; Smitka et al., 1992, Park et al., 1992; Schwartz et al., 1987; Mo et al., 2010). The types of indole alkaloids and the genera that the compounds were isolated from are shown in Table 2. An example of the base structures of these indole alkaloids is given in Figure 2. As a result of the variability in the structures of these compounds the variant were organized into ten groups. 
Table 2: Distribution of Indole Alkaloids in the Family Stigonemataceae

\begin{tabular}{|c|c|c|c|c|}
\hline & Fischerella & Hapalosiphon & Westiella & Westiellopsis \\
\hline Fischerindoles & $\mathbf{X}$ & $\mathbf{X}$ & & \\
\hline Hapalindoles & $\mathbf{X}$ & $\mathbf{X}$ & & \\
\hline Ambiguines & $\mathbf{X}$ & $\mathbf{X}$ & & $\mathbf{X}$ \\
\hline Welwitindolinones & & $\mathbf{X}$ & $\mathbf{X}$ & \\
\hline Hapalindolinones & $\mathbf{X}$ & $\mathbf{X}$ & & \\
\hline Oxidized Welwitindolinones & $\mathbf{X}$ & & & \\
\hline Fischambiguines & $\mathbf{X}$ & & & \\
\hline
\end{tabular}




(1)

Figure 2: Indole Alkaloid from Stigonemataceae (Stratmann et al., 1994; Smitka et al., 1992; Mo et al., 2010; Papke et al., 1997) 


\subsubsection{Hapalindoles}

Out of all of the indole alkaloids produced by the family Stigonemataceae, the hapalindoles are the largest group and are classified in Groups 1 and 2 in Figure 2. The base structure can be either tricyclic in the case of Group 2 or more commonly tetracyclic as with Group 1 (Moore et al., 1987). The $\mathrm{R}^{2}$ functional group is either chlorine or hydrogen and these chlorinated compounds are commonly isolated with the corresponding non-chlorinated analogues. The $\mathrm{R}^{1}$ functional group is usually either an isonitrile or an isothiocyanate, but with Hapalindole $\mathrm{T}$ this functional group is a thiocarbamate group (Figure 3).

Hapalindoles A and B were originally isolated from Hapalosiphon fontinalis by Moore et al., (1984). The examination of two sympatric cyanobacteria Hapalosiphon intricatus and Anabaena by Moore et al., (1984) showed the inhibition of the growth of Anabaena. In the same investigation, similar observations were reported for Hapalosiphon fontinalis which sparked an interest in allelochemicals from this species (Moore et al., 1984). Lipophilic extracts of $H$. fontinalis produced antimycotic and antialgal activity and two novel bioactive compounds, Hapalindoles A and B, were isolated from this crude extract. Hapalindoles A is a tetracyclic, chlorinated; isonitrile containing indole alkaloid and Hapalindole B is the corresponding isothiocyanate. Both Hapalindole A and B would be classified under Group 1. Hapalindole A was shown to be the main bioactive compound from the lipophilic extract. Two oxidation products of Hapalindole A, Fontonamide and Anhydrohapoxindole A, were also isolated from a more polar fraction of Hapalosiphon fontinalis (Moore et al., 1987). Singlet oxygen oxidation of Hapalindole A produced a possible precursor of the oxidative products labeled 
Hapalonamide A along with Fontonamide and Anhydrohapoxindole A. Eighteen other hapalindoles, Hapalindoles $\mathrm{C}-\mathrm{Q}$ and $\mathrm{T}-\mathrm{V}$, have been isolated from $H$. fontinalis by Moore et al., (1987), and are shown in Figure 3 with Hapalindoles A and B. Hapalindoles C-Q and $\mathrm{T}-\mathrm{V}$ were not isolated using bioassay guided fractionation so the bioactivity of these compounds was not addressed in these studies. Hapalindole $\mathrm{T}$ was also isolated from Fischerella sp. by Asthana et al., (2005), and in this study the bioactivity of the hapalindole was investigated. Hapalindole $\mathrm{T}$ was shown to have antibacterial properties with an MIC value of $2.5 \mu \mathrm{g} / \mathrm{mL}$ towards Staphylococcus aureus, and $2.0 \mu / \mathrm{mL}$ towards Pseudomonas aeruginosa. At a concentration of $2.5 \mu \mathrm{g} / \mathrm{mL}$, Hapalindole $\mathrm{T}$ was also shown to be active against Mycobacterium tuberculosis with inhibition comparable with the ant-TB drug Rifampicin. 


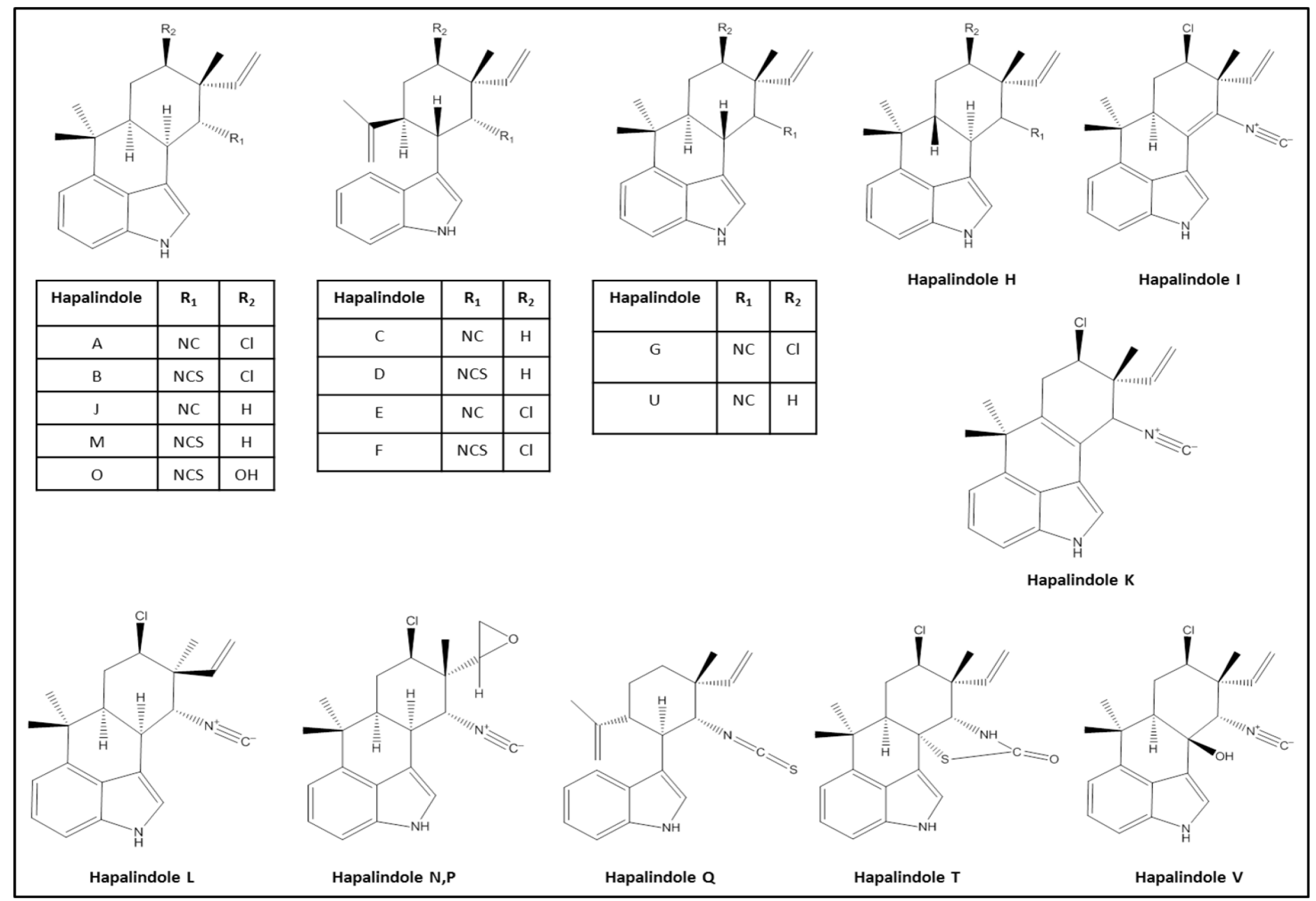

Figure 3: Hapalindole Structures Moore et al., (1987) 
Epimers of many of these hapalindoles, with a reversed stereochemistry at the $\mathrm{C}(12)$ position, have also been isolated. The alkaloid 12-epi-Hapalindole $\mathrm{E}$ was originally isolated by Schwartz et al., (1990) from Fischerella (ATCC 53558). This compound has also been isolated from Hapalosiphon welwitschii, Hapalosiphon laingii, and Fischerella JAVA 94/20 (Doan et al., 2000; Klein et al., 1995). The compound 12epi-Hapalindole E Isonitrile had been reported to have a range of bioactivities that include algicidal, insecticidal, fungicidal activities, and the ability to inhibit RNA polymerase. A similar compound was isolated from Hapalosiphon laingii and Fischerella JAVA 94/20 (12-epi-Hapalindole H Isonitrile). An isolation of compounds from $H$. laingii by Klein et al., (1995) not only produced the two hapalindole epimers, 12-epi-Hapalindole $\mathrm{H}$ and $\mathrm{E}$ Isonitrile, but also 12-epi-Hapalindoles $\mathrm{C}, \mathrm{G}$, and $\mathrm{Q}$ Isonitriles. The Klein et al., (1995) study also afforded two hapalindolinones, Hapalindolinone A and B (Group 9), and unfortunately the bioactivities of the individual compounds were not addressed. The algicide 12-epi-Hapalindole $\mathrm{F}$ was isolated from Fischerella CENA 19, a strain of Fischerella found in the Amazon River in Brazil (Etchegaray et al., 2004). The latest investigation of novel hapalindoles was a study by Becher et al., (2007), where insecticidal compounds from Fischerella (ATCC 43239), a bio-film forming strain, was identified and isolated. In this study a novel epimer, 12-epiHapalindole J Isonitrile, was identified with three other previously identified hapalindoles including 12-epi-Hapalindoles C and E Isonitrile and Hapalindole L. At concentrations ranging from $26-37 \mu \mathrm{M}$ each of these compounds was shown to cause $100 \%$ mortality of the larvae of the Harlequin fly in 48 hours. Many epimers containing an isonitrile group in the structure have been isolated; however, two epimers with the isothiocyanate group 
were isolated from Hapalosiphon welwitschii by Stratmann et al., (1994). These compounds were 12-epi-hapalindole F and D Isothiocyanate. The bioactivities of these compounds were not addressed in the study.

\subsubsection{Hapalindolinones}

Hapalindolinones A and B were originally isolated from Fischerella isolated from a soil sample from the Florida Everglades (Schwartz et al., 1987). The unique structure, which contains a "spiro-fused cyclopropane ring", had not yet been documented from the indole alkaloids produced by Stigonemataceae (Schwartz et al., 1990). From Figure 2 the hapalindolinones are categorized in Group 9. As with the hapalindoles the $\mathrm{R}^{2}$ group is either chlorine or hydrogen and the $\mathrm{R}^{1}$ group is an isonitrile group. Hapalindolinone $\mathrm{B}$ is the non-chlorinated version of Hapalindolinone A. Of the two structures, Hapalindolinone A was shown to moderately inhibit $\left[{ }^{3} \mathrm{H}\right]$ arginine vasopressin binding to kidney tissue with an IC50 of $37.5 \pm 7.6 \mu \mathrm{M}$, and kidney (v2) arginine vasopressin stimulated adenylate cyclase with an IC50 of $44.6 \mu \mathrm{M}$.

\subsubsection{Ambiguines}

The second largest group of indole alkaloids isolated from Stigonemataceae is the ambiguines. Similar in structure to hapalindoles, the ambiguines have an isoprene unit attached at the $\mathrm{C}(2)$ position of the indole moiety (Figure 2), and the variability of the ambiguine structure occurs with the cyclization of this group (Smitka et al., 1992; Mo et al., 2009; Raveh and Carmeli 2007). Accordingly, these indole alkaloids are organized in Group 3 for the tetracyclic ambiguines, and Group 4 and 10 for the pentacyclic ambiguines (Figure 2). The pentacyclic ambiguines of Group 5 have also been shown to contain a diol, a ketone, or a double bond in place of the epoxide group. In Groups 3 and 
4 the $\mathrm{R}^{2}$ group again is defined as the chlorine or hydrogen group, but $\mathrm{R}^{1}$ is either a hydroxyl group or hydrogen. All of the ambiguines in Groups 3 and 4 contain isonitrile and so far no isothiocyanate containing ambiguines have been isolated. Group 10 ambiguines are unique in that the $\mathrm{R}^{1}$ group can either be hydrogen in the case of Ambiguine $\mathrm{P}$ or a nitrile group in the case of Ambiguine $\mathrm{Q}$. The occurrence of the nitrile or lack an isonitrile is very different from the majority of the indole alkaloids from Stigonemataceae which normally include an isonitrile or isothiocyanate. Chlorinated and non-chlorinated versions of these compounds occur at the $\mathrm{R}^{2}$ position. Like some of the other ambiguines, these structures can also contain a hydroxyl group which would occur at the $\mathrm{R}^{3}$ position.

From a study by Smitka et al.,(1992) six novel indole alkaloids, Ambiguines A-F Isonitriles, were isolated, which include both Group 3 and 4 ambiguine structures. These ambiguines were isolated from crude extracts of Fischerella ambigua, Hapalosiphon hibernicus, and Westiellopsis prolifica that showed antifungal activity. Ambiguine isonitriles A-F were isolated from Fischerella ambigua, Ambiguine A and E were isolated from Hapalosiphon hibernicus, and Ambiguines D and E were isolated from Westiellopsis prolifica. The main fungicidal activity was produced by Ambiguine Isonitriles A and E. Ambiguines A-F showed some activity towards Candida albicans with MIC values ranging from 1.25 to $2.5 \mu \mathrm{g} / \mathrm{mL}$. The fungicidal activity of the ambigiunes was not comparable to the commercial fungicide amphotericin B which produced an MIC value of $0.156 \mu \mathrm{g} / \mathrm{mL}$. Using the same assay conditions, when Ambiguines A-F were tested against Aspergillus fumigatus the MIC values ranged from 0.625 to $20 \mu \mathrm{g} / \mathrm{mL}$ which again was not comparable to the commercial fungicide 
tolnaftate which produced an MIC value of $<0.02 \mu \mathrm{g} / \mathrm{mL}$. Five other ambiguine isonitriles from Group 4 were isolated from Fischerella ambigua which included Ambiguine K-O Isonitrile (Mo et al., 2009). Ambiguine K Isonitrile showed moderate activity towards Mycobacterium tuberculosis with an MIC value of $6.6 \mu \mathrm{M}$. All of these compounds were isolated on the basis of antimicrobial activity. Ambiguine K-N Isonitriles were shown to also be moderately active against Candida albicans with MIC values ranging from 1.1 to $<0.9 \mu \mathrm{M}$.

Ambiguines H-J Isonitriles were isolated from Fischerella sp. From a soil sample collected in Israel (Raveh and Carmeli 2007). Like Ambiguines K-O, these ambiguine structures are from Group 4. Ambiguine $\mathrm{H}$ and $\mathrm{I}$ isonitrile showed antibacterial and antifungal activity comparable with commercial antibacterial and antifungal agents streptomycin and puramycin/amphotericin B. Ambiguine I Isonitrile was shown to be more potent compared to Ambiguine H Isonitrile by producing lower MIC values.

The first ambiguines to be isolated all contained an isonitrile group, however, a paper written by Huber et al., (1998) showed the isolation of a nitrile containing ambiguine named Ambiguine G Nitrile from Hapalosiphon delicatulus. Of the indole alkaloids identified from Stigonemataceae this was the first alkaloid that contained a nitrile group. The bioactivity of this compound was not investigated as a result of the nature of the fractionation. Most of these alkaloids are isolated on the basis of some observed bioactivity but Ambiguine G Nitrile was isolated by spectral analysis. The nitrile containing ambiguines have a structure classified under Group 10 which contains the polyunsaturated ring attached to the $\mathrm{C}(2)$ position of the indole moiety. A paper later published by Mo et al., (2010) showed the isolation of a new nitrile containing compound 
Ambiguine Q Nitrile from Fischerella ambigua. In this same study a new ambiguine was isolated that did not contain either a nitrile or isonitrile group called Ambiguine P. Ambiguine P and Ambiguine Q Nitrile showed weak antimicrobial activity.

\subsubsection{Fischambiguines}

From the study previously discussed, two new indole alkaloids were isolated with novel tetracyclic structure which is depicted in Figure 2. Fischambiguines A and B were isolated from Fischerella ambigua by Mo et al., (2010) and were shown to have antibacterial activity. The bioactivity was not comparable to the positive controls. These indole alkaloids under Group 5 include either a chlorine or hydrogen at the $\mathrm{R}^{2}$ position and a methylene or epoxide group as the R group.

\subsubsection{Fischerindoles}

Fischerindoles were first isolated from Fischerella muscicola by Park et al., (1992) in a study that screened cyanobacteria for antifungal activity. This isolation produced Fischerindole L. Fischerindoles are categorized into Group 6 in Figure 2. The tetracyclic structure of these compounds is similar to the structure of the tetracyclic hapalindoles in Group 1 except for the additional double bond in the six membered ring and the bonding at the $\mathrm{C}(2)$ position in the indole moiety. As with the hapalindoles, the $\mathrm{R}^{1}$ group is either an isonitrile or an isothiocyanate, and the $\mathrm{R}^{2}$ group is either chlorine or hydrogen. The compound called Fischerindole $\mathrm{L}$ was named on the basis of the similarity in the stereochemistry of the molecule Hapalindole L, also isolated in this study. The structures of each of these compounds are depicted in Figure 4 . Fischerindole L Isonitrile was shown to have antifungal properties similar to Hapalindole L Isonitrile. 


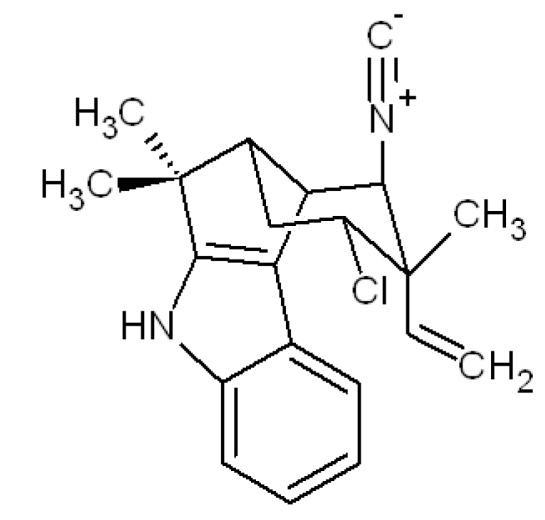

Fischerindole L

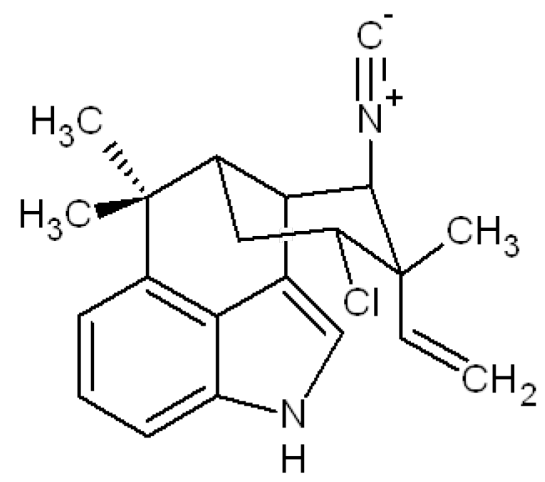

Hapalindole L

Figure 4: Structural Comparison of Fischerindole L and Hapalindole L. (Park et al., 1992)

Epimers of the fischerindoles have also been isolated with similar confirmations at the $\mathrm{C}(12)$ position as the hapalindoles. Four other fischerindoles were isolated from Hapalosiphon welwitschii named 12-epi-Fischerindole I Isonitrile, 12-epi-Fischerindole $\mathrm{U}$ Isonitrile and the corresponding isothiocyanate, and 12-epi-Fischerindole G Isonitrile (Stratmann et al., 1994). As with Fischerindole L Isonitrile, the nomenclature of these compounds was generated from the similarities of the stereochemistry of the fischerindoles to the hapalindoles. However, unlike the structure depicted in Figure 2, 12-epi-Fischerindole $\mathrm{G}$ isonitrile and 12-epi-Fischerindole $\mathrm{U}$ isonitrile, and the corresponding isothiocyanate, are lacking the double bond on the six-membered ring. The bioactivity of these compounds was not addressed in this study.

\subsubsection{Welwitindolinones}

An interesting group of indolinone containing compounds called welwitindolinones was isolated from Westiella intricata and Hapalosiphon welwitschii 
by Stratmann et al., (1994). In Figure 2 these compounds are categorized into two groups, Group 7 and 8. Group 7 includes only Welwitindolinone A, a chlorinated, isonitrile containing structure with the unique cyclobutane group. The remaining welwitindolinones are classified under Group 8 which contains far greater structural variability. All of the welwitindolinones are chlorinated and have either an isonitrile or an isothiocyanate at the $\mathrm{R}^{2}$ position. Some of these structures are also methylated at the $\mathrm{R}^{1}$ position. The $\mathrm{R}^{3}$ position is hydrogen except for the oxidized welwitindolinones which have a hydroxyl group. Welwitindolinone $\mathrm{C}$ isothiocyanate, the corresponding methylated indole nitrogen with either the isothiocyanate and isonitrile contain an additional double bond at the chlorine substituted carbon.

From the Stratmann et al., (1994) study, Welwitindolinones B and C Isothiocyanate and N-Methylwelwitindolinone $\mathrm{C}$ Isothiocyanate were isolated from Westiella intricata. The observed larvicidal activity against blowfly larvae of the lipophilic extract of Westiella intricata led to the isolation of the three welwitindolinones. The primary larvicidal compound was determined to be N-Methylwelwitindolinone C Isothiocyanate, also called welwistatin, which also showed MDR reversing properties. In a later study it was shown by Zhang et al., (1996) that welwistatin was also an antimicrotubule compound. These compounds were also isolated from Hapalosiphon welwitschii along with Welwitindolinone A Isonitrile, N-Methylwelwitindolinone D Isothiocyanate, 3-epi-Welwitindolinone $\mathrm{B}$ Isothiocyanate, and NMethylwelwitindolinone C Isothiocyanate (Stratmann et al., 1994). Welwitindolinone A Isonitrile was also shown to be fungicidal. 
Oxidized products of welwitindolinones have been isolated from Fischerella muscicola and Fischerella major (Jimenez et al., 1999). One new welwitindolinone, NMethylwelwitindolinone D Isonitrile, and two oxidized welwitindolinones, 3-HydroxyN-methylwelwitindolinone Isonitrile and Isothiocyanate, were isolated from Fischerella sp. It was shown that photooxidation of the previously isolated N-Methylwelwitindoline C Isonitrile produced N-Methylwelwitindolinone D Isonitrile and 3-Hydroxy-Nmethylwelwitindolinone D Isonitrile.

An extract from a single cyanobacterial strain can contain a variety of these compounds, many of which containing two to three types of these indole alkaloids (Stratmann et al., 1994). The observed isolation of multiple similar indole alkaloids from a single source leads to the conclusion that a similar biosynthetic pathway occurs for these compounds.

\subsection{Biosynthesis of Indole Alkaloids from Stigonemataceae}

A common biosynthesis was proposed that utilized the same precursor molecules and produced the variety of indole alkaloid structures isolated. A biosynthesis of these compounds was proposed by Stratmann et al., (1994). In this biosynthetic route the main base molecule for the chlorinated, isonitrile-containing indole alkaloids was 12-epiHapalindole E Isonitrile. Stratmann proposed that the synthesis of this molecule occurs by a chloronium ion-induced condensation of 3-(Z-2'-Isocyanoethenyl)Indole and (Z)3.7-dimethyl-1,3,6-octatriene as shown in Figure 5. The formation of 12-ep-Hapalindole E Isonitrile is suspected to occur from the derivation of geranyl pyrophosphate and tryptophan (Park et al., 1992). Each of these precursors has been shown to occur in other organisms (Evans et al., 1976; Adekunle et al., 2007). The precursor, 3-(Z-2'- 
Isocyanoethenyl)Indole has been isolated from the bacterium Pseudomonas, and the precursor (Z)-3,7-dimethyl-1,3,6-octratriene is a volatile terpene found in higher flora. The precursor 3-(Z-2'-Isocyanoethenyl) Indole, also called Antibiotic B371, was isolated from Pseudomonas from the muddy water weeds in Harefield, Middlesex, England (Evans et al., 1976). Antibiotic B371 was shown to have MIC value of $8 \mu \mathrm{g} / \mathrm{mL}$ against Staphylococcus aureus in an agar diffusion assay. The precursor (Z)-3.7-dimethyl-1,3,6octatriene was hypothesized to be a derivative of geranyl pyrophosphate. Geranyl derivatives like geranylacetone have been shown to be produced by cyanobacteria like Microcystis (Ikawa et al., 2001).

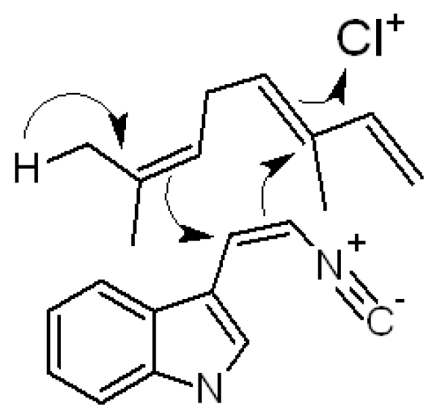

$\mathrm{H}$

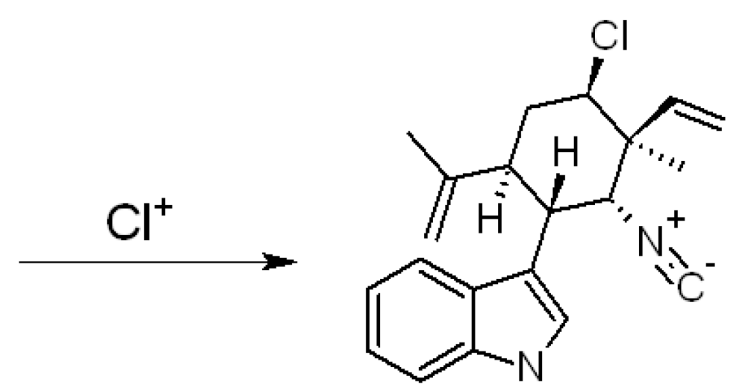

$\mathrm{H}$

1 (12-epi-hapalindole E Isonitrile)

Figure 5: Biosynthesis of 12-epi-Hapalindole E Isonitrile (Richter et al., 2008) 


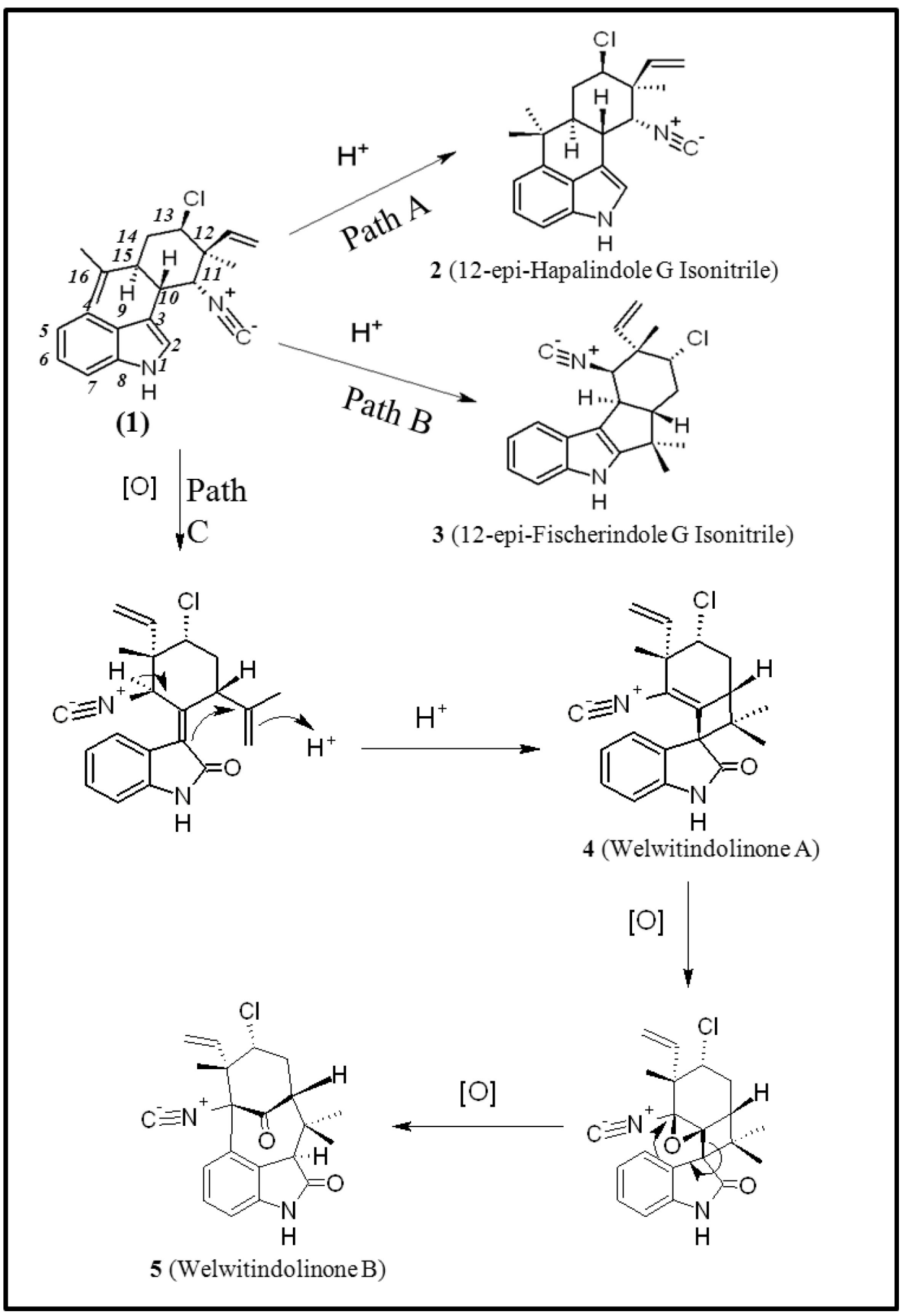

Figure 6: Biosynthesis of Hapalindoles, Fischerindoles, and Welwitindolinones (Richter et al., 2008)

After the formation of 12-epi-Hapalindole E Isonitrile the biosynthetic scheme diverges into three paths as shown in Figure 6 (Richter et al., 2008). The first path 
suggests the cyclization between the $\mathrm{C}(4)$ carbon in the indole ring and the $\mathrm{C}(16)$ carbon in the isopropylidene group in 1 (Richter et al., 2008). The cyclization reaction would produce the tetracyclic hapalindoles of Group 1, namely 12-epi-Hapalindole G Isonitrile, 2. The formation of oxindoles and formamides could occur through oxidation and oxidative cleavage of these tetracyclic hapalindoles. If an isoprene group is attached to $\mathrm{C}(2)$ of the indole ring, the indole alkaloids from Group 3 are formed. However, if the isoprene group is then reacted further by intermolecular cyclization this would lead to the pentacyclic ambiguines of Group 4. Oxidation and rearrangement of these compounds afford other varieties of the ambiguine family which makes up Groups 5 and 10.

The second path proposes the cyclization of 12-epi-Hapalindole E Isonitrile between $\mathrm{C}(2)$ of the indole ring and $\mathrm{C}(16)$ of the isopropylidene group producing the tetracyclic fischerindoles of Group 6, namely 12-epi-Fischerindole G Isonitrile, 3. The condensation of the hapalindole would occur via acid catalysis and possibly governed by an enzyme. Park et al., (1992) had shown that the cyclization reaction was possible without the presence of an enzyme with Hapalindole C Formamide. A strong acid was used to convert Hapalindole $\mathrm{C}$ Formamide to Fischerindole $\mathrm{C}$ Formamide and Fischerindole C Amine (Bonjouklian et al., 1988). Using the same conditions, this same reaction was not able to be reproduced with Hapalindole E Formamide, which differs from Hapalindole C Formamide by a chlorine atom. It is possible that a different catalyst would drive the condensation.

The third path suggests the oxidation of 12-epi-Hapalindole E Isonitrile to produce an intermediate indolinone with a structure similar to Anhydrohapaloxindole A. Anhydrohapaloxindole A has been shown to be able to be produced from singlet oxygen 
oxidation of hapalindole A (Moore et al., 1989). This intermediate then undergoes intermolecular cyclization between $\mathrm{C}(3)$ of the indole ring and $\mathrm{C}(16)$ of the isopropylidene group via acid catalysis to produce welwitindolinone A 4 from Group 7. In order to form the remaining welwitindolinones like 5 from Group 8, an isocyano epoxide intermediate is formed and further intermolecular rearrangements occur. Oxidation and methylation of the welwitindolinones produces the oxidized welwitindolinones, for example, 3-hydroxy-N-methylwelwitindolinone C Isonitrile.

The proposed biosynthetic route suggests the common synthesis of these alkaloids; however, there were two discrepancies in this hypothesis. The occurrence of non-chlorinated and chlorinated versions of the same compounds has been attributed to the possible competition at the condensing enzyme active site between proton and chlorine ions (Raveh and Carmeli 2007). Since the chlorine does not play a role in the cyclization processes, the formation of non-chlorinated indole alkaloids like hapalindoles, ambiguines, fischerindoles, and welwitindolinones would occur. However, the isolation of non-chlorinated welwitindolinones has not been reported, and may be as a result of the lack of bioactivity of these compounds. In the paper by Jimenez et al., (1999), oxidized welwitindolinones were isolated using spectral analysis. In the study only chlorinated oxidized welwitindolinones were isolated. If these compounds were synthesized from the precursors using a chloronium ion induced condensation to form a tricyclic hapalindole structure that would later produce the welwitindolinones, then a non-chlorinated version of these compounds would have to exist. Another issue with the proposed biosynthetic process is the lack of addressing the biosynthesis of the cyclopropane alkaloids the 
hapalindolinones of Group 9. The unique structure variability of these compounds may indicate an alternative synthetic pathway.

The biosynthetic origin of the isonitrile group that occurs in many of these compounds has been investigated with Hapalindole A from Hapalosiphon fontinalis by Bornemann et al., (1987). In his feeding experiment it was shown that the highest percent of the radio-labeled species that was incorporated into Hapalindole A as the isonitrile was [2-14C]Glycine, L-[3-14C] Serine, and [14C]Cyanide. It was shown that the labeled $14 \mathrm{C}$ and $15 \mathrm{~N}$ from the labeled glycine were incorporated as the isonitrile group. The observed incorporation leads to the hypothesis that the formation of the isonitrile group is plausible as a result of the tetrahydrofolate (THF) metabolism. The carbon and the nitrogen could have been taken up by THF to form an intermediate, 5formamino-THF, which then allowed the donation of the carbon and nitrogen to the indole containing molecule. The incorporation of inorganic cyanide was not addressed in the present study. However, it was proposed that the formation of the cyanide was possible and could be as a result of the oxidation of amino acids (Knowles et al., 1976). The formation of the HCN through amino acid oxidation has been reported with bluegreen algae Anacystis nidulans (Pistorius et al., 1979; Pistorius et al., 1980). Bornemann et al., (1987) also proposed the uptake of the cyanide in the THF metabolism to serve as a substrate for the formation of the formamino-THF derivative. Potassium cyanide was fed to the biomass but proved to be toxic to the cyanobacteria. Similar feeding experiments with sponges have also shown that inorganic cyanide can be incorporated in isonitrile natural products (Garson and Simpson 2004). Feeding experiments where Amphimedon terpenensis were fed [14C]-labeled inorganic cyanide showed that the cyanide was 
incorporated into the isonitrile groups of the natural product diisocyanoadociane. The process for incorporating the cyanide was suggested to be a result of an enzymatic process since incorporation does not occur without the sponge tissue. Since it has been shown that cyanobacteria can be found in sponges, it is possible that these organisms play a symbiotic role with the sponge and may be responsible for some of the metabolites produced (Arillo et al., 1993).

The formation of the isothiocyanate group is not as clearly studied as the isonitrile group for these compounds. It has been proposed that the sulfur could have been incorporated into the organic isonitrile intermediate or an inorganic source of thiocyanate was incorporated (Stratmann et al., 1994). The subsequently formed intermediate 3-((Z)2'-isothiocyanatoethenyl)indole would then undergo the chloronium ion-induced condensation reaction. A number of feeding studies with sponges have been done to determine the origin of the isothiocyanate in marine natural products produced by sponges (Simpson and Garson 2004). From these studies it was observed that cyanide and thiocyanate were incorporated into the isothiocyanate group of the natural product 9isothio-cyanatopupukeanane by the sponge Axinyssa (Simpson et al., 1998). In a similar study labeled thiocyanate was fed to sponge tissue from $A$. terpenensis, and was incorporated into the isonitrile group of the natural product diisocyanoadociane (Simpson et al., 1999). Alternatively, labeled cyanide has been shown to be incorporated into the isothiocyanate groups of 9-isothiocyanatopupukeanane by Axinyssa. These studies show that certain enzymatic processes allow the interconversion between the cyanide and thiocyanate or the isonitrile and isothiocyanate groups (Garson and Simpson 2004). This 
could explain why many of these isonitrile containing indole alkaloids are isolated with the isothiocyanate analogues.

Unlike the isonitriles and isothiocyanates, the nitrile group is only found in the ambiguines of Group 10. The nitrile is proposed to occur by a rearrangement of an isonitrile ambiguine (Huber et al., 1998). The isonitrile is proposed to migrate and reverse polarity to produce the nitrile (Van Wagoner et al., 2007).

\subsection{Structure-Activity Relationship of Indole Alkaloids from Stigonemataceae}

The variation in structures has produced a range of bioactivities. However, from the limited data on the bioactivity of many of these compounds, it seems that the isonitrile containing alkaloids have more documented cases of bioactivity (Moore et al., 1987; Stratmann et al., 1994; Smitka et al., 1992). The isolation of many hapalindoles by Moore et al., (1987) was done using TLC analysis and was not guided by any bioassay. Therefore, the bioactivity of the isonitrile or isothiocyanate groups of most of these compounds was not addressed. However, the original isolation of Hapalindole A Isonitrile showed that the compound was responsible for the observed antialgal and antimycotic activity of the crude material (Moore et al., 1987). Isonitrile containing ambiguines have also been shown to have antimicrobial activity; these include Ambiguines K and M. (Mo et al., 2009). Many of the isonitrile containing ambiguines also have fungicidal properties which include Ambiguine A through F (Smitka et al., 1992). Welwitindolinone A Isonitrile was shown to have fungicidal properties (Stratmann et al., 1994). The isonitrile containing Fischerindole L was shown to have antifungal properties (Park et al., 1992). Isonitrile containing compounds from each type 
of indole alkaloid have shown some form of bioactivity all of which include some form or microbial or fungal activity.

Other isonitrile containing marine natural products have shown to be quite bioactive as antimalarial compounds (Garson and Simpson 2004). The isonitrile prevents the conversion of iron porphyrins to $\beta$-hematin by binding to the porphyrins, and the membranes of the malarial parasites are then destroyed by the build-up of heme moieties. None of the indole alkaloids from Stigonemataceae have been investigated as antimalarial compounds.

Interestingly there is not much information on the bioactivity of the isothiocyanate containing indole alkaloids. One welwitindolinone, NMethylwelwitindolinone C Isothiocyanate, was shown to have insecticidal properties and the ability to reverse MDR. Hapalindole B was isolated in small amounts along with Hapalindole A and was not reported to have any bioactivity (Moore et al., 1984). Whether this observation is a result of a concentration factor or lack of bioactivity against microbes and fungi is unknown. Other non-isonitrile containing indole alkaloids have also been reported to be bioactive. The thiocarbamate hapalindole, Hapalindole T, was also shown to be antimicrobial as previously discussed (Asthana et al., 2006).

The bioactive compounds; Fischerindole L, Hapalindole A Isonitrile, 12-epiHapalindole E Isonitrile, Welwitindolinone A, N-Methylwelwitindolinone C Isothiocyanate, and many ambiguines also contain chlorine groups. The role of chlorine in the bioactivity of these compounds was addressed by Raveh and Carmeli (2007). They have discussed the bioactivity of chlorinated and non-chlorinated ambiguines and the lack of any difference in antimicrobial activity for the either kind of compound (Raveh 
and Carmeli 2007). The evaluation of bioactive chlorinated hapalindoles versus the nonchlorinated hapalindoles has only been addressed with the insecticidal bioactivity observed with 12-epi-Hapalindole J, C, and E Isonitrile and Hapalindole L. Both the non-chlorinated and chlorinated isonitrile containing hapalindoles were shown to be larvicidal towards the harlequin fly larvae at concentrations between 26 and $37 \mu \mathrm{M}$ (Becher et al., 2007). On the basis of data generated by Becher et al., (2007) and Raveh and Carmeli (2007) the chlorinated hapalindoles and ambiguines produce comparable bioactivities. Most likely the chlorine does not play much of, if any role in the fungal and microbial activity.

One compound, 12-epi-Hapalindole E Isonitrile had been investigated using a variety of bioassays and had shown to produce a range of bioactivities (Doan et al., 2000). This compound contains both chlorine and isonitrile groups. When tested against algae, fungi, bacteria, and mammalian cells this compound was shown to have an inhibitory effect in the $\mu \mathrm{M}$ dose range for Myeloma (Mouse), the green algae Monoraphidium convolutum, and various bacteria, cyanobacteria, and fungi. The actual mode of action was determined to be the inhibition of RNA and protein synthesis. The inhibition was determined to be a result of interaction between 12-epi-Hapalindole E Isonitrile and RNA polymerase (Doan et al., 2000). The hapalindole was shown to compete with ATP for the binding site on the enzyme substrate on E. coli RNA polymerase. The observed activity was most likely not the only cellular process that was being affected, since the concentration levels to observe inhibition of purified RNA polymerase were higher than the levels seen with cellular growth inhibition (Doan et al., 2001). A separate instance of isolating insecticidal compounds from Fischerella also 
produced 12-epi-Hapalindole E Isonitrile (Becher et al., 2007). It is clear that this compound affects a basic biological process that leads to a variety of bioactivities. With many of these indoles containing a similar base structure to 12-epi-Hapalindole E Isonitrile, it would be useful to investigate the full range of bioactivity for other indole alkaloids. The analysis the bioactivities of the indole alkaloids previously isolated might shed some light on which structures of the compounds are bioactive and could be utilized to improve natural product screening from cyanobacteria. In order to obtain a more wide scope of the nature of these compounds, a more complex assay would be useful.

In the family Stigonemataceae at least four genera have been shown to produce these indole alkaloids. Literature search for hapalindole-like compounds did not yield any results outside the cyanobacterial family Stigonemataceae. The use of these compounds has not been determined, but the various forms of bioactivity suggest that the genera from Stigonemataceae pose as a good source of biologically relevant chemicals. 


\section{TOXICOLOGICAL AND CHEMICAL INVESTIGATION OF FISCHERELLA 52-1 USING THE ZEBRAFISH (Danio rerio) EMBRYO MODEL}

\subsection{Introduction}

Fischerella, a genus in the family Stigonemataceae, has been shown to produce a number of indole alkaloids as discussed in Chapter 1. A screening of extracts from different cyanobacteria isolated from the Florida Everglades for bioactivity yielded one strain of Fischerella that was shown to be very bioactive (Berry et al., 2007; Gantar et al.,2008). Fischerella 52-1 produced a unique consistent set of physical deformities in developing zebrafish embryos, and this strain was shown to inhibit growth of cocultivated algae. Initial isolation of the active compound suggested an indole alkaloid (unpublished data) (Berry et al., 2007). In order to identify active compounds from Fischerella, the zebrafish (Danio rerio) embyo model was used to guide the purification of bioactive compounds.

\subsection{Zebrafish (Danio rerio) as a Toxicological Model}

The zebrafish model has been utilized for a variety of purposes which include but are not limited too genetic research, toxicology, drug discovery, and developmental biology (Hill et al., 2005; Crawford et al., 2008). Using the zebrafish model, many bioactive metabolites from cyanobacteria have been evaluated (Berry et al., 2008 and 2009 and Wright et al., 2006). The zebrafish embryo model has many advantages that make it a useful tool in evaluating bioactivity of crude material and guiding the purification of bioactive metabolites (Berry et al., 2008). One specific benefit of this model is the wide range of bioactivities that are able to be detected. The ability arises as a result of the many biological processes that occur during embryogenesis, and when any 
one of these processes is affected the embryo does not develop correctly. The abnormal development can then be used to guide the purification of the bioactive compound(s). Other advantages of the zebrafish embryo assay include easy husbandry, a transparent chorion, small size, and rapid embryogenesis. The small size, around $1 \mathrm{~mm}$ in diameter, and transparent chorion, or outer "shell" of the zebrafish eggs, allows the embryos to be studied light microscopy. The small size permits a high throughput assay to be performed in 24 to 96 wellplates, which can accommodate a substantial quantity of extracts or fractions per assay. The ease of husbandry ensures that a large number of eggs can be produced by a relatively small number of breeding pairs. The rapid embryogenesis allows multiple assays to be performed in a small window of time, between 3 to 5 days. The zebrafish embryo assay has been performed using two different exposure methods (Berry et al., 2007 and 2009). One includes injecting the material of interest into the zebrafish embryos using a microsyringe or a nanoliter injector, and in the other method the extracts or toxins are introduced in the rearing media. The second method subjects the embryos to the test solution by immersion in ambient concentrations.

The bioactivity of several cyanobacterial toxins and extracts have been investigated using the zebrafish embryo model (Lefebvre et al., 2004; Oberemm et al., 1999; Berry et al., 2004, 2007, and 2009). Oberemm et al., (1999) tested purified compounds, Microcystins LR and RR (MC-LR and MC-RR) and saxitoxin, from cyanobacteria and crude cyanobacterial extracts against a range of aquatic vertebrate embryo models including zebrafish embryos. In the study performed by Oberemm et al., (1999), the toxins and extracts were introduced to the zebrafish through the rearing medium. Microcystin LR and RR, in the $\mu \mathrm{g} / \mathrm{L}$ concentrations, showed reduced survival 
and growth weight of the zebrafish embryos. Exposure to higher levels of the microcystins, as high as $10 \mathrm{mg} / \mathrm{mL}$, to the zebrafish through the rearing medium showed that the elevated concentrations of MC-LR produced developmental defects, which included a pectoral edema and a distended yolk sac.

Even though it has been shown that MC-LR did not produce much bioactivity, except at high concentrations, when the zebrafish embryo was exposed through the rearing media, a paper by Wang et al., (2005) suggested that the weak bioactivity observed with the MC-LR was as a result of the lack of uptake of the toxin by the embryo. To determine whether a different exposure method could cause a change in the degree of bioactivity, the zebrafish embryos were injected with MC-LR via microinjection at the connection of the yolk cell and the blastodisc. The MC-LR was injected to insure that the toxin was introduced into the embryo directly, and was not relying on cellular uptake from the surrounding environment with the MC-LR only available in the cytoplasm. Embryos injected at the 2-4 cell stage post fertilization were shown to suffer from abnormal blastomeres, at the 8 cell stage. Wang et al., (2005) compared the tightly fixed blastomeres that occur in normal embryos to the embryos dosed with MC-LR, and discovered an absence of organization which subsequently led to increased mortality. In this study it was also shown that a dose and time-dependent mortality trend was observed. Embryos that were able to survive past the 48 hours post fertilization (hpf) point experienced various deformities. These defects included cyclopia, pericardial edema, malformation of the tail, edema of the hatching gland, and curvature of the body axis. The use of the microinjection method produced a greater bioactivity compared to the ambient exposure method. Similar increased bioactivity has 
been shown for other microinjected cyanobacterial toxins which were not observed when the toxin was introduced via the rearing medium (Berry et al., 2009). The low degree of bioactivity produced by the cyanobacterial toxins when exposed to the zebrafish embryos in the rearing medium may be a result of the high water solubility of these toxins, or the inability of these toxins to pass effectively through the chorion. The hydrophilic character of the molecule might not be the only reason toxins similar to MC-LR was not able to diffuse through the chorion. The size of the large cyclic peptide might have some effect on the ability of the molecule to cross into the embryo.

Saxitoxin, which causes paralytic shellfish poisoning, is a toxin that is produced by a dinoflagelletes and cyanobacteria and has also been evaluated with the zebrafish embryo model (Oberemm et al., 1999; Lefebvre et al., 2004; Sivonen et al., 1996). Oberemm et al., (1999) reported the effects of a Saxitoxin concentration of $500 \mu \mathrm{g} / \mathrm{L}$, which was shown to produce embryos with abnormal body axis curvature and edema. These defects were shown to be reversible upon the immersion of the embryos in toxin free medium. In the study by Lefebvre et al., (2004), Saxitoxin was introduced into the embryos through the egg rearing medium with increasing concentrations. This paper helped to confirm the ability of zebrafish to uptake a toxin through the rearing media. Physical morphologies observed included edema of the pericardium, eye, and yolk sac and abnormal curvature of the body axis. Sensory abnormalities were also observed which included postponed or diminished touch response and paralysis. It was suggested by Lefebvre et al., (2004) that the edema was most likely caused by the paralysis which impeded the ability of the embryo to balance fluids and osmotic pressures. Some of the embryos were able to regain normal touch response and reverse the paralysis once 
removed to a toxin-free medium. The microinjection method was not required in the two Saxitoxin studies previously discussed, since the molecule was able to diffuse through the chorion of the developing zebrafish embryo. There is a possibility that the smaller size of Saxitoxin compared to MC-LR and MC-RR could play a role in the ability for Saxitoxin to be taken up by the embryos.

The cyanobacterial toxin $\beta$-N-Methylamino-L-alanine (BMAA), and a glutamate analog produced by mixing BMAA and carbonate, were also investigated using the zebrafish embryo model in a paper by Purdie et al., (2009). In this study the zebrafish embryos were exposed to rearing media that contained increasing amounts of BMAA, and BMAA with sodium carbonate, at 24 hpf. After five days of exposure the embryos were transferred to new rearing media that was free of BMAA and observed for recovery. As with many of the previous zebrafish assays, morphological abnormalities were observed which included pericardial edema and curvature of the body axis. The embryos exposed to only the BMAA showed some pericardial edema, but the embryos exposed to both BMAA and carbonate produced a trend of increasing number of embryos with edema with increasing amounts of BMAA and carbonate. The occurrence of the curved spinal cord was increased with the BMAA, and BMAA with carbonate, compared to the controls, but no dose dependent trend was observed. A significant amount of involuntary muscle contractions and premature hatching was also observed in embryos exposed to BMAA with carbonate. It was suggested that the convulsions might have some effect on the integrity of the chorion leading to the premature hatching. The study by Oberemm et al., (1999), discussed in the previous sections, exposed the zebrafish embryos to other water soluble toxins, like Saxitoxin and MC-LR and MC-RR, using the ambient exposure 
method. Lower levels of bioactivities were observed in the Oberemm et al., (1999) study compared to the Purdie et al., (2009) study. It is possible that the relatively small size of BMAA might allow this molecule to pass into the cytoplasm of the embryo.

Pahayokolide A, a compound isolated from Lyngbya, was shown to produce mortality in the zebrafish embryo assay (Berry et al., 2004). The method of exposure was through dissolution in the rearing media. Mortality of $100 \%$ of the embryos was observed at $24 \mathrm{hpf}$ at a concentration of $5 \mu \mathrm{g} / \mathrm{mL}$ and at 5 days post fertilization (dpf) at a concentration of $3 \mu \mathrm{g} / \mathrm{mL}$. This compound, however, did not produce any developmental defects. The large size of this molecule compared to MC-LR and MC-RR would suggest that the bioactivity would be minimal if the size of the molecule influenced the diffusion process of the toxin through the chorion. However, given the high mortality observed with Pahayokolide $\mathrm{A}$ it is possible that a portion of the molecule passes through the chorion and produces the observed bioactivity.

The cyanobacteria Aphanizomenon flos-aquae was found in the Mueggelsee in Berlin and analyzed for cyanotoxin content by Papendorf et al., (1997). It was shown that the cyanobacteria were producing microcystins at low concentrations which was later attributed to possible small amounts of Microcystis sp. When an extract of the A.flosaquae was tested against zebrafish embryos, unusually pronounced developmental retardation was observed that could not be correlated to microcystin. Fractionation and purification produced two compounds, Mueggelone and Lupenyl Acetate. The zebrafish embryo assay, using the ambient exposure method, was not used to guide the fractionation, but was used to evaluate the bioactivity of the purified compounds. The assay revealed that $10 \mu \mathrm{g} / \mathrm{mL}$ of Mueggelone caused mortality of $45 \%$ of embryos and 
severely retarded the development of the surviving embryos. Lupenyl Acetate, at a concentration of $100 \mu \mathrm{g} / \mathrm{mL}$, showed pericardial edema and bent tails. In this case, the ambient exposure through the rearing media allowed new bioactive compounds to be detected that might not have been otherwise identified.

Other harmful toxins produced by other organisms have also been evaluated using the zebrafish embryo model (Tiedeken et al., 2005; Lefebvre et al., 2004). Domoic Acid, which results in amnesic shellfish poisoning, is a toxin produced by diatoms unlike many of the toxins previously discussed (Tiedeken et al., 2005). A study by Tiedeken et al., (2005) investigated the use of the zebrafish embryo assay whereby the toxin was microinjected into the zebrafish embryo. From the assay it was observed that as the concentration of Domoic Acid increased the hatching success rate decreased. At the higher concentrations certain morphological abnormalities were observed, which included pericardial edema, downward curvature of the spinal column, and underdevelopment of the jaw. These deformities were observed in most of the unhatched embryos exposed to high concentrations of Domoic Acid. It was suggested that the defects were not consistent nor were expressed by all exposed embryos. Neurotoxic and behavioral effects were also observed and included convulsions, lack of escape response in the presence of a stimulus, and continuous pectoral fin movements. A dose-dependent trend was observed with embryos exposed to increasing amounts of Domoic Acid. As concentration increased, embryos exhibited neurological defects, abnormal swimming behavior, and paralysis. The bioactivity observed with Domoic Acid may be dependent on the susceptibility of the embryo to the toxin which would account for the mixture of observations. 
Another cyanobacterial toxin, the hepatotoxic Cylindrospermopsin (CYN), was analyzed using the zebrafish embryo model by Berry et al., (2009). In this study five exposure methods were used to evaluate the toxicological effect of pure CYN on the zebrafish embryos, and different cyanobacterial extracts, from the corresponding genera Cylindrospermopsis and Aphanizomenon, were evaluated using the assay. The different exposure methods for purified CYN included, microinjection into the yolk cell adjacent to the blastomeres, and ambient exposure including, partial dechorionation, use of distilled water as an egg rearing medium, and addition of DMSO to normal rearing solution. From this experiment it was observed that microinjection was the only methods that produced any bioactivity, and that a dose-dependent mortality, with abnormal development, was observed. Surviving embryos were shown to yield an assortment of developmental defects with no discernible pattern. In the same study, different cyanobacterial extracts from Cylindrospermopsis raciborski and Aphanizomenon ovalisporum were tested using the zebrafish embryo assay, specifically using the ambient exposure method. From this experiment it was observed that consistent patterns of developmental defects formed, a given concentration of lipophilic extracts of these species produced the same defect in all of the embryos exposed. These deformities included truncated or curved body axis, pericardial edema, and diminished development of the eye. Delayed hatching was also observed with the exposed embryos. Most notably, developmental defects were observed with chloroform extracts of both CYN and non-CYN producing strains. It was observed that the exposure to the crude material produced a consistent set of deformities that were expressed by all embryos in the exposed well. 
One of the key observations in the study by Oberemm et al., (1999), was also the difference in activity in zebrafish embryos exposed to purified compounds, including microcystin, and those exposed to a crude extract. More pronounced developmental defects were observed in embryos exposed to a crude extract from different cyanobacterial species than embryos exposed to microcystins alone. Interestingly the higher levels of deformity were produced by embryos exposed to extracts of Microcystis aeruginosa that contained little to no microcystin as determined by High Performance Liquid Chromatography. Some of the defects from the exposure to the crude extract of M. aeruginosa included pericardial edema, truncated and curved body axis. Extracts of Aphanizomenon flos-aquae caused the softening and rupturing of the chorion of the zebrafish embryo. The observation of more pronounced developmental defects from crude extracts compared to the purified toxins produced by the same cyanobacteria may indicate a synergistic effect produced by the exposure of multiple compounds or additional unidentified bioactive compounds.

One particular point of interest with these toxin exposure experiments is the lack of uniform phenotypes. The term "phenotype," which is defined as the physical expression of an organisms genes, does not truly define the observed deformities in the exposure experiments since it is not known whether a particular pathway or the genes are being affected by the toxin (Raven and Johnson 1990). The novel term "toxitype" can be used to define the common physical expression of the effects of a toxin on all exposed embryos (Pat Gibbs personal communication, March 13, 2009).

In the previously discussed cases, pure cyanobacterial toxins were not shown to cause uniform deformities in a particular exposure, however a common toxitype was 
observed with embryos exposed to crude extracts from various cyanobacteria (Berry et al., 2004, 2007, and 2009; Lefebvre et al., 2004, Oberemm et al., 1999, Papendorf et al., 1997, Purdie et al., 2009, Tiedeken et al., 2005, and Wang et al., 2005). Many of the toxins previously discussed mainly caused mortality and some developmental defects, but it was not discussed whether the exposure caused reproducible consistent defects. Some common deformities observed in the ambient exposure, and the microinjection experiments, were pericardial edema and curved body axis. The common occurrence of these deformities had not been addressed; however the edema, in one case, was attributed to paralysis of the embryo (Lefebvre et al., 2004).

The zebrafish model has also been shown to be a useful tool in screening for bioactive compounds from cyanobacteria in a study by Berry et al., (2008). In this study, polar and nonpolar extracts from different cyanobacterial strains were screened against the zebrafish embryo model to explore new bioactivity. Bioactivity in the polar, $30 \%$ ethanol, extracts mostly produced deaths among the embryos but the nonpolar; chloroform extracts produced developmental defects in live embryos. The occurrence of a consistent toxitype in exposed zebrafish embryos was observed in the lipophilic chloroform extracts of some of the cyanobacteria tested (Berry et al., 2008). Specifically, the extract of Fischerella 52-1 was shown to produce the toxitype termed "puffy" in embryos exposed to the crude lipophilic extracts of the biomass and the culture medium. Initial characterization of one of the active compounds suggests the active compound belonged to the family of secondary metabolites that included many indole alkaloids, discussed in Chapter 1. 
Other bioactive metabolites of Fischerella have been identified using the zebrafish embryo model (Wright et al., 2006). A study by Wright et al., (2006) showed that when zebrafish embryos were exposed to the culture medium of Fischerella ambigua, via ambient exposure, 100\% mortality was observed, and when this extract was diluted the embryo development was significantly stunted which eventually caused embryo mortality. Fractionation of the active medium identified Ambigol A and C, and a small amount of Tjipanazole D discussed in Chapter 1. When the embryos were exposed to each of the purified compounds, only high concentrations of Ambigol A produced any activity. The activity occurred at $1 \mathrm{mg} / \mathrm{mL}$, and caused irregular shape of the body and tail and pectoral edema. High Performance Liquid Chromatography analysis of the culture medium showed that the concentration of Ambigol A was $0.47 \mathrm{mg} / \mathrm{mL}$, much less than the amount required to produce bioactivity. When compared to the observed activity from the culture medium it is apparent that the purified compounds were not as potent separately compared collectively. When the fractions were recombined the activity could not be reproduced leading to the conclusion that an additional unidentified metabolite, was potentially present and acted synergistically with Ambigol A. The Wright et al., (2006) study showed that bioactive compounds were possible to detect and purify from cyanobacteria like Fischerella using the zebrafish embryo model. The analysis of the culture medium, confirmed that bioactive metabolites could also be isolated from the culture medium and not solely the cellular material. The observation of bioactivity in the zebrafish embryos from a cyanobacterial culture medium was also reported for Fischerella 52-1 by Berry et al., (2008). 


\subsection{Use of the Zebrafish Embryo Model to Investigate Bioactive Metabolites from Fischerella 52-1.}

Extracts of the cyanobacterium Fischerella 52-1 has been documented to produce a number of bioactivities (Berry et al., 2007; Gantar et al., 2008). Screening of cyanobacteria collected from central and south Florida by Gantar et al., (2008), investigated the interactions or allelochemistry of cocultivated algae and cyanobacteria, and the effects of the cyanobacterial extracts. The most potent isolate was Fischerella 52-1 which was shown to inhibit the growth of all tested green algae and cyanobacteria. Extracts of Fischerella 52-1 from the biomass, and the culture medium, were investigated and the extracts from the culture medium were shown to be more potent. The effect of the culture medium extract of Fischerella 52-1 on the green algae Chlamydomonas was investigated further. The extract exhibited a concentration and time dependent trend of increasing photosynthesis inhibition, along with an observed decrease in the organization of the cellular structure of Chlamydomonas. The morphological abnormalities observed with exposed Chlamydomonas included bleaching of the cells, swollen membranes, deterioration of thylakoids, and disappearance of some organelles like the nucleus. It was suggested from this study that the active compound, as a result of the lipophilic nature of the active extracts, might target the membranes of the thylakoids which are lipid-rich, and this might cause an interruption of the electron transport system.

The bioactivity of the lipophilic extract of Fischerella 52-1 was also explored with the zebrafish embryo model. The initial interest in Fischerella 52-1 stemmed from the study performed by Berry et al., (2008), where the "puffy" toxitype was first observed in the lipophilic extract of Fischerella 52-1 and the culture medium. 
Interestingly there was a more potent effect from exposing the embryos to the extracted culture medium of Fischerella 52-1. As with the algal bioassay, the zebrafish embryo bioassay showed more activity in the extract from the culture medium compared to the extract from the biomass. Because of this observation, it was suggested that secondary metabolites were possibly produced by the strain and released into the culture medium (Berry et al., 2007).

Furthermore, the culture of Fischerella 52-1 produced a globular solid that surrounded the cellular material and culture flask perimeter. Exocellular material has been shown to occur in some other strains of cyanobacteria, but the nature of its occurrence is unclear (Philippis et al., 1998). One hypothesis is that the material acts like a buffer between the cyanobacterial colony and its environment. The material could produce antialgal or antifeedant compounds to protect the colony or limit desiccation by storing water and releasing it slowly. The exocellular material is possibly composed of secondary metabolites, which allows the inhibition of predation or the propagation of competing species.

The different bioactivities observed with Fischerella 52-1 suggest that this cyanobacterium could produce multiple bioactive compounds (Berry et al., 2007; Gantar et al., 2008). Since the zebrafish embryo model showed such pronounced activity towards this extract, this model was used to guide the purification of the active compound in this research. The evaluation of the "puffy" toxitype in each facet of the Fischerella 52-1 culture, including the unique globular material or extracellular solid (ECS), was investigated using the zebrafish embryo assay. The active fractions and purified compounds were analyzed using mass spectrometry and HPLC-UV-vis. 


\subsection{Methods}

\subsubsection{Fischerella 52-1 Culture}

Fischerella 52-1 was isolated from the Florida Everglades by Gantar et al., (2007). Identification of the taxon was determined using a 16S rRNA gene sequencing followed by a BLAST search and was determined to be Fischerella, and the strain was labeled 52-1. The strain was cultured as per Berry et al., (2007). These conditions required the strain to be grown in BG 11 medium, buffered with 2morpholinoethanesulfonic acid (MES) to $\mathrm{pH} 7.2$ at $24^{\circ} \mathrm{C}$ under constant fluorescent white light. Biomass, culture medium, and ECS of Fischerella 52-1 was obtained from nonaxenic unialgal cultures.

\subsubsection{Extraction}

Each component of the culture including the culture medium, biomass, and ECS was extracted for subsequent evaluation. The culture medium was extracted by $\mathrm{C} 18$ solid phase extraction (SPE). Two Restek reverse-phase SPE cartridges with 5g C18 bonded silica, and $20 \mathrm{~mL}$ sample volume, were used to extract two 4-L samples of culture medium, and subsequently eluted off with methanol. Two grams of freeze-dried biomass were extracted twice with $200 \mathrm{~mL}$ of $1: 1$ propanol and dichloromethane, as specified by Moore et al., (1987), on a shaker table overnight. The material from each of the two extractions was combined. Since the active compound was thought to be a hapalindole, the same extraction process was used to extract the cellular material as the original hapalindole isolation (Berry et al., 2007; Moore et al., 1987)). The ECS was collected, by Dr. Gantar, from the culture by scraping the material from the biomass of the growing culture and culture flask into a $20 \mathrm{~mL}$ scintillation vial. The material was extracted 
overnight on a shaker with $10 \mathrm{~mL}$ of hexane. The hexane extract was removed, and the material was subsequently extracted with acetone. The hexane and acetone extracts were tested against the zebrafish. The observed bioactive acetone extract was used to compare the bioactivity of the ECS to the culture medium and biomass extracts using the zebrafish embryo assay.

\subsubsection{Optimized Extraction of ECS}

Analysis of purified materials showed that the solubility of the compounds of interest was low for solvents like acetone, ethyl acetate, and hexane. However, the compounds were shown to be quite soluble in methanol. Because of this observation, the extraction procedure for the ECS was optimized by exchanging the overnight hexane and acetone extractions for two overnight methanol extractions.

\subsubsection{HPLC Comparison of Bioactive Components of Fischerella 52-1 Culture}

A crude fractionation was performed on each of the initial bioactive extracts from the Fischerella 52-1 culture; these included the extracts from the biomass, culture medium, and ECS. The fractionation was performed using a silica solid phase extraction (SPE) cartridge with a solvent system ranging from 100\% hexane, 5:1, 4:1, 3:1, 2:1, 1:2 hexane to acetone, and $100 \%$ acetone. Bioactive fractions from each fractionation were then evaluated by the HPLC on a Shimadzu Prominence UFLC system, with a PDA detector. A Luna C18 analytical column with dimension of $250 \times 4.60 \mathrm{~mm}$ and particle size of $5 \mu \mathrm{m}$ with $100 \AA$ pore size was used. The method utilized a flowrate of $1 \mathrm{ml} / \mathrm{min}$, and included a smooth gradient with acetonitrile and water. The starting concentration of acetonitrile was $50 \%$ and was increased to $60 \%$ over 10 minutes. The concentration was 
kept constant for 5 minutes. The concentration of acetonitrile was increased to $70 \%$ over 5 minutes, and this concentration was kept constant for an additional 10 minutes. The concentration was then increased to $100 \%$ over five minutes and kept at a constant concentration for 5 minutes.

\subsubsection{Fractionation of ECS}

Two sequential HPLC methods were used to purify indole alkaloids from the optimized methanol extracts of ECS using a Shimadzu Prominence UFLC system, with a PDA detector to separate the indole containing compounds from the active extract. The indole chromophore observed in the active fraction had a $\lambda_{\max }$ at $220-225$ and $278-282$ $\mathrm{nm}$. The chromatographic methods were developed to separate the observed peaks with a $\lambda_{\max }$ at $222 \mathrm{~nm}$. Each method utilized a Luna Semi-Prep C18 column with $5 \mu \mathrm{m}$ particle size and dimensions of $250 \times 10 \mathrm{~mm}$ with a flowrate of $4.5 \mathrm{~mL} / \mathrm{min}$. The first HPLC method used an acetonitrile and water gradient. The gradient began at 50\% acetonitrile and ended at $99 \%$ acetonitrile in 20 minutes, and an isocratic gradient at $99 \%$ acetonitrile was kept for an additional 20 minutes. The last segment of the method was added after the analysis of the gradient, using a PDA detector that was used in sequence with a mass spectrometer, showed that compounds not detected by the PDA were observed after the 20 minute gradient. The indoles of interest were collected at 15 to 20 minutes, this was determined after a smaller scale collection was done and tested against the zebrafish embryo model. The collected fraction at 15-20 minutes was determined to be bioactive and was further fractioned using a second method. The second method used a very slight gradient of methanol and water which started with $73.2 \%$ methanol and was increased to $73.5 \%$ over the span of 40 minutes. The percent of methanol was again increased to $75 \%$ 
for the following 12 minutes which afforded eight different peaks with indole-like chromophores. Six fractions containing either a single large peak or a group of small peaks were collected and tested against the zebrafish embryo assay. The fractions containing peaks 7 and 8 were determined to be pure, but the fraction containing peak 3 required further purification using the HPLC. A third method was developed that utilized a 25 minute isocratic method with a 53\% acetonitrile concentration. The Luna C18 analytical column was used in this method at a flowrate of $1 \mathrm{ml} / \mathrm{min}$.

\subsubsection{Zebrafish Embryo Bioassay}

The zebrafish embryo assay was performed as per Berry et al., (2007). The zebrafish used to test the quantified crude material were the L3 line, the original purification methods used the L4 and L5 lines, and the rest of the analysis was done using the P5 line. The assay utilized polypropylene 24-well plates which contained dried cyanobacterial material. Solvent controls, cyanobacterial extracts, HPLC fractions, and solutions of purified compounds from Fischerella 52-1 were pipetted into wells, and allowed to dry overnight in a fume hood. Fertilized zebrafish eggs were obtained from Dr. Pat Gibbs at the University of Miami Rosenstiel School of Marine and Atmospheric Sciences at Key Biscayne, FL. To obtain eggs, 50 pairs of zebrafish were bred in 4 10-L tanks containing a mesh to separate breeding adults from eggs. The eggs are collected from the tanks, washed, and rinsed with the E3 medium. The E3 medium was made using a 60x stock E3 solution and $0.1 \%$ methylene blue solution. The 60x stock solution was made by dissolving $8.6 \mathrm{~g} \mathrm{NaCl}, 0.38 \mathrm{~g} \mathrm{KCl}, 1.45 \mathrm{~g} \mathrm{CaCl}_{2} * \mathrm{H}_{2} \mathrm{O}$, and $1.2 \mathrm{~g} \mathrm{MgSO}_{4}$ in $500 \mathrm{~mL}$ of ultrapure water. The E3 solution required $8 \mathrm{~mL}$ of the $60 \mathrm{x}$ stock and $250 \mu \mathrm{L}$ of the $0.1 \%$ methylene blue solution to make $500 \mathrm{~mL}$ of $\mathrm{E} 3$ media. Following the rinsing, 
the eggs at the 32 to 64 cell stage were sorted from the unfertilized eggs, poor quality eggs, and day 1 embryos. The assay utilized five fertilized embryos at the $32-64$ cell stage. Eggs were pipetted with $1 \mathrm{ml}$ of E3 medium into each well. The bioactivity produced by the material in each well was compared to negative controls, and documented 3-5 days post fertilization (dpf); the first indication of the toxitype produced by Fischerella 52-1, occurred at 3 days post fertilization. Observations and images were taken using an Olympus SZX7 dissecting microscope, with internal camera.

Crude extracts of the biomass, culture medium, and the ECS were dried and weighed to allow a concentration to be calculated and used to quantify the toxicity in terms of $\mu \mathrm{g}$ residue/mL solution. In order to determine which extract of the ECS to use, a small zebrafish embryo assay was performed using the hexane and acetone extract. When zebrafish embryos were exposed to each fraction, the acetone fraction was shown to produce the "puffy" toxitype, and the hexane fraction was no longer utilized. To evaluate the activity of the extracts against the zebrafish embryo model, a bioassay was setup where 6 wells with differing concentrations of dried residue from each extract that included $0,10,25,50,100,250 \mu \mathrm{g}$ residue/ $\mathrm{mL}$ of E3, were evaluated with the zebrafish embryos. Five embryos were exposed to the dried residue in the $1 \mathrm{~mL}$ of E3 medium. Solvent controls with dried hexane and acetone produced no mortality or abnormal developmental defects when exposed to the zebrafish embryos.

Similarly purified amounts of Peak 3,7 , and 8 were analyzed using the zebrafish embryo assay and included the concentrations, $0,0.1,0.5,1,5$, and $10 \mu \mathrm{g} / \mathrm{mL}$ of E3. In order to measure the amount of each toxin, a pre-weighed HPLC vial, without the cap, was filled with a solution of the desired toxin in methanol. The cap was returned to the 
vial and the solvent was removed by introducing filtered dry air through a needle that was pierced through the septa cap and allowing the air to vent through an additional needle. The vial was not allowed to come in contact with any surface which was executed by covering the outside of the vial with a Kimwipe. Once the solvent was evaporated the cap of the vial was removed, and then weighed to determine the amount of toxin. In order to make a stock solution $1 \mathrm{~mL}$ of methanol was then introduced into the vials with known amounts of toxin and a desired amount was removed to make a stock solution. For Peak 3 the stock concentration was $25 \mathrm{ug} / \mathrm{mL}$, for Peak 7 the stock concentration was $33 \mu \mathrm{g} / \mathrm{mL}$ and for Peak 8 the stock concentration was $53 \mu \mathrm{g} / \mathrm{mL}$. Different volumes of each stock were pipetted into wells of a 24-well plate to give the desired concentrations.

\subsubsection{Embryo Removal Experiments}

In order to assess the recovery from toxic effects, at $3 \mathrm{dpf}$ of the zebrafish embryo assay, with the quantified amounts previously discussed of Peaks 3, 7, and 8, one embryo was removed from each of the desired bioactive wells. Each embryo was photographed and then removed from the chorion and photographed again. The removed embryos were then placed into new wells in a 24-well plate containing $1 \mathrm{~mL}$ of only the $\mathrm{E} 3$ medium. The same removal method was repeated on 4 and 5 dpf with embryos from each of the same bioactive wells. The removed embryos were observed and photographed each day after removal. Two embryos were left in the well containing the cyanobacterial material to serve as positive controls.

\subsubsection{Mosquito Larvicidal Assay}

In addition to the zebrafish embryo assays, extracts and purified compounds were evaluated for mosquito larvicidal activity. The mosquito larvicidal assay was performed 
by Gerald Berry using his procedure described below. A 24 well plate was used containing dried cyanobacterial extracts or purified compounds in each well. The extracts were resuspended in $10 \mu \mathrm{L}$ of methanol followed by $500 \mu \mathrm{L}$ of water. The plate was then placed on a shaker table for 3hours to allow evaporation of the methanol. For this assay mosquito larvae, Aedes aegypti of the Rockefeller strain, were hatched in $250 \mathrm{~mL}$ jars with $75 \mathrm{ml}$ of water previously rendered hypoxic by boiling to $50 \%$ of the volume. A small piece of paper containing the desired amount of mosquito eggs was then added to the jar and sealed. The eggs were allowed to hatch for 2 hours and the larvae were transferred to a petri dish. Four larvae with $400 \mu \mathrm{L}$ of water were then pipetted into each well following the solvent removal. After the all of the desired larvae were transferred into the 24 wellplate, $120 \mu \mathrm{L}$ of $0.1 \%$ autoclaved liver powder solution was pipetted into each well. The plates were observed for bioactivity each day after hatching for 1 week. The bioactivity investigated using light microscopy included mortality, delayed development, and any other deformities. Mortality was determined by a three stage analysis, first by observing lack of movement, then by agitating the larvae, and finally by light microscopy.

\subsubsection{LC-MS of Indoles from ECS}

Purified compounds and fractions were evaluated by the LC-MS using a TSQ Quantum Access LCMS system. A Heated Electrospray Ionization (HESI) source was used both in negative and positive modes. A methanol and water gradient from $70 \%$ to $99 \%$ methanol in water, with a flowrate of $500 \mu \mathrm{L} / \mathrm{min}$, over the span of 28 minutes was used on a Kinetex C18 column ( $100 \times 4.6 \mathrm{~mm}$, and particle size $2.6 \mu \mathrm{m})$. Each scan was 
evaluated to determine $\mathrm{m} / \mathrm{z}$ of the purified molecule, and to determine relative purity of the sample.

\subsubsection{Toxin Solubility Analysis}

Two sets of six wells, in two 24-well plates, were used to evaluate the solubility of the purified Peaks 7 and 8 in E3 medium. The six amounts of each toxin, that were dispensed twice in six different wells in each of two rows, included $0,0.1,0.5,1,5$, and $10 \mu \mathrm{g}$. Quantification of the purified material was done using the same method described above for the analysis of purified toxins. Once the appropriate amount of each toxin was distributed into the 24 wells, the plates were allowed to dry overnight in the hood. To determine the relative solubility of each toxin in the E3, compared to the methanol which has shown to facilitate complete dissolution at these concentrations, one row was then suspended in $1 \mathrm{~mL}$ of methanol, and the other row was suspended in $1 \mathrm{~mL} \mathrm{E3}$. Aliquots of each set of six samples were analyzed using the TSQ Quantum Advantage Mass Spectrometer. The method used to detect each peak was composed of a full scan and a single ion monitoring (SIM) scan set for the mass of each peak using a methanol and water gradient previously discussed. Each method was performed using a HESI probe set to a negative polarity for Peak 7, and a positive polarity for Peak 8 . The column used was a Kinetex C18 column, previously discussed, at a flowrate of $500 \mu \mathrm{L} / \mathrm{min}$ and the gradient included 30 minute increase of methanol concentration from $30 \%$ to $99 \%$. The areas of the chromatogram peaks correlating to the SIM scan were used to compare the amount of toxin dissolved in methanol, and the amount dissolved in the E3. 


\subsection{Results and Discussion}

\subsubsection{Toxicological Investigation of Crude Extracts}

Embryos exposed to the biomass, culture medium, ECS and extracts showed developmental dysfunctions including well conserved toxitypes and ranges of effects. As a result of the uniqueness of the developmental defects from Fischerella 52-1 observations of the development were taken at $2 \mathrm{dpf}$ up until $5 \mathrm{dpf}$. At $1 \mathrm{dpf}$ the dosed embryos do not appear any different from the controls, it is not until 2 dpf that color variation and edema begin to be apparent. At $5 \mathrm{dpf}$ all embryos should be hatched, but it was observed that embryos dosed with small amounts of the crude material developed normally but did not hatch even at $7 \mathrm{dpf}$. In order to serve as a comparison the normal development of the zebrafish is depicted in Figure 7, and control images will be shown with each experiment. 


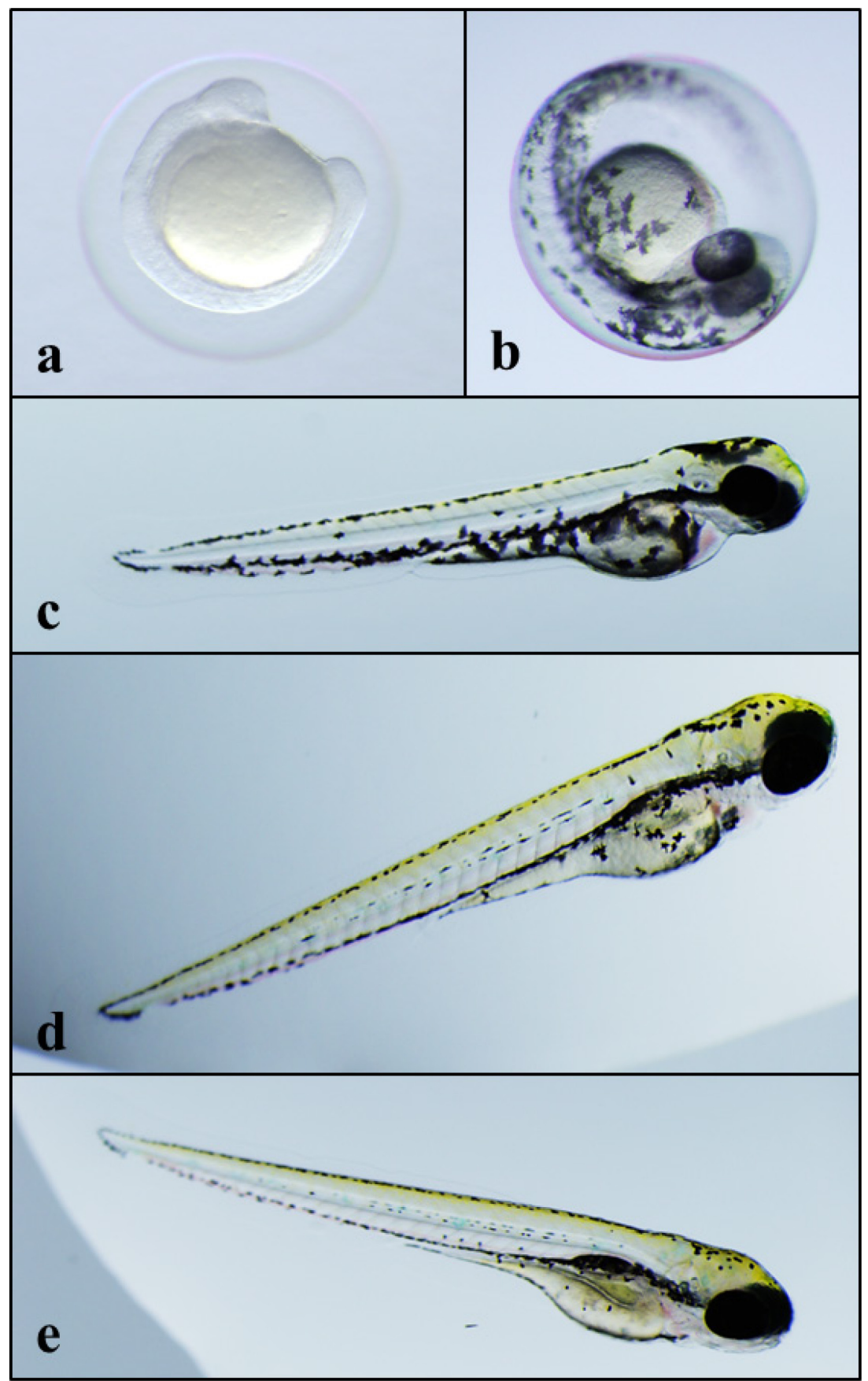

Figure 7: Normal Zebrafish development; a) Day 1, b) Day 2, c) Day 3, d) Day 4, and e) Day 5

In order to determine which part of the culture was bioactive each component was extracted and tested on the zebrafish embryo assay. The results of the zebrafish embryo assay, using the crude extracts, are shown in Table 3 and Figure 8. From Table 3 it is 
shown that the ECS produced activity at a much lower mass per volume concentration which indicates the possible occurrence of an increased concentration of an active compound or compounds. Alternatively this could also indicate the occurrence of a strong bioactive effect of a synergistic mixture of compounds. The acetone extract from the ESC was shown to produce the "puffy" toxitype with the least amount of the crude material. From this observation, it was decided that the ECS would be the main focus for further purification.

Table 3: Results of Zebrafish Embryo Bioassay on Crude Material

\begin{tabular}{|c|c|}
\hline Extract & $\begin{array}{c}\text { Lowest Concentration with } \\
\text { Observed Bioactivity }\end{array}$ \\
\hline Cellular Material & $250 \mu \mathrm{g} / \mathrm{mL}$ \\
\hline Culture Medium & $250 \mu \mathrm{g} / \mathrm{mL}$ \\
\hline Extracellular Solid & $10 \mu \mathrm{g} / \mathrm{mL}$ \\
\hline
\end{tabular}




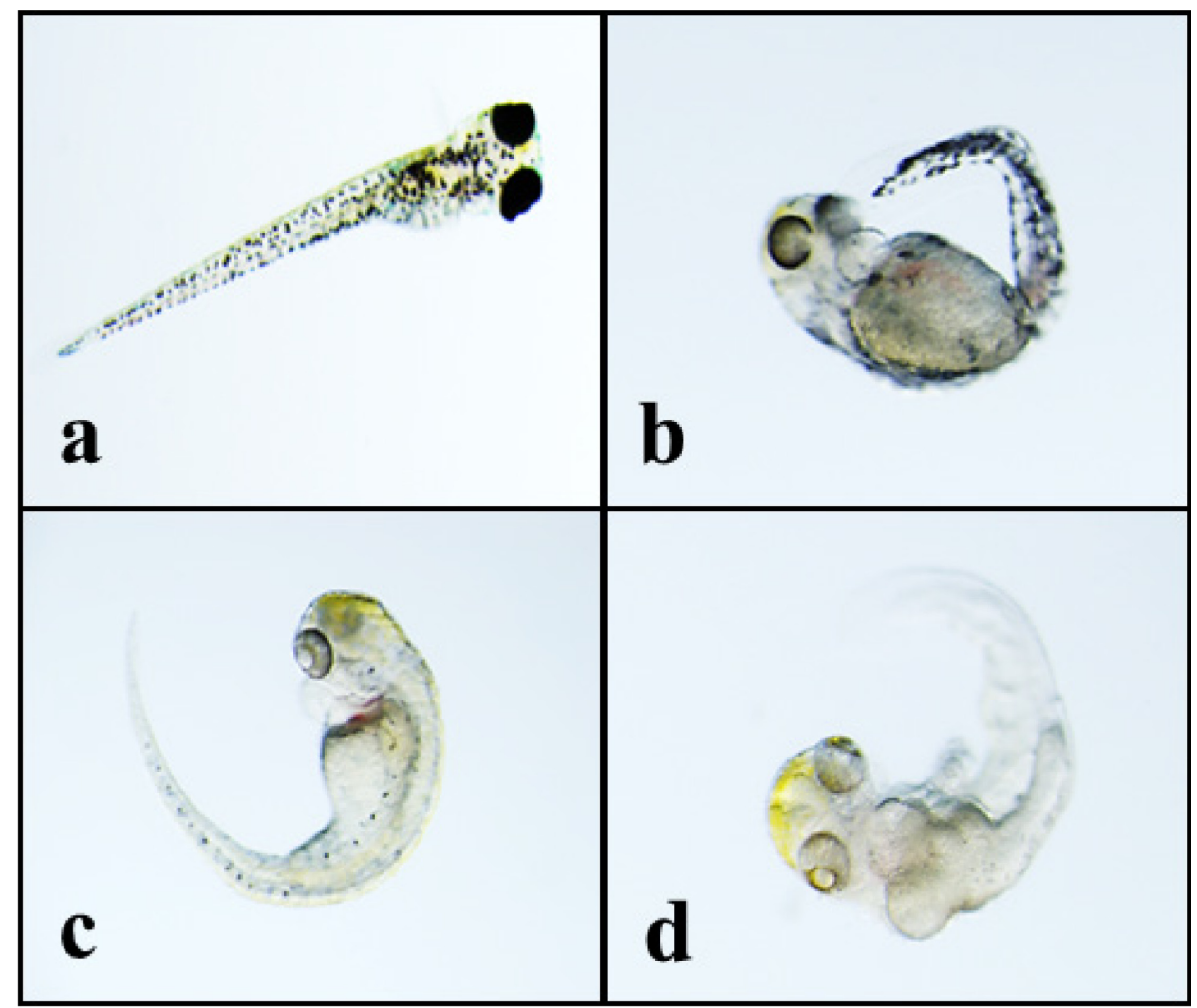

Figure 8: Fish Embryo Bioassay Observations 5 dpf; a) Control, b) Biomass $250 \mu \mathrm{g} / \mathrm{mL}$, c) Culture Medium 250 $\mu \mathrm{g} / \mathrm{mL}$, d) ECS $10 \mu \mathrm{g} / \mathrm{mL}$

The crude fractions of the bioactive extracts from the Fischerella 52-1 biomass, culture medium, and ECS, using the silica SPE cartridge, were tested against the zebrafish embryo assay. The "puffy" toxitypes developed in the 5:1 hexane to acetone fraction from each component of the culture. Analysis of each component of the culture using HPLC revealed that apparently similar compounds, containing indole chromophores, were observed (Figure 9). The indole chromophore was identified on the basis of the UV $\lambda_{\max }$ from other indole alkaloids from Fischerella and related genera that are categorized under the family Stigonemataceae, discussed in Chapter 1 (Moore et al., 1987, Smitka et al., 1992 and Stratmann et al., 1994). The documented $\lambda$ max for these indole alkaloids occurred from 219-225nm and 275-282nm and sometimes with a 
shoulder at $290 \mathrm{~nm}$. As an example, Figure 10 is a spectrum obtained from the HPLC of one collected indole alkaloid from Fischerella 52-1. For each component of the culture of Fischerella 52-1, the HPLC chromatogram showed a cluster of indole chormophores in the time range between 26 and 28 minutes. In this range two large peaks each containing an indole chromophore was observed (Figure 9). In each component the first of these peaks was smaller than the second, but in the culture medium the ratio of the first peak to the second peak was higher. Most likely a larger amount of a single compound or multiple compounds are exuded into the surrounding environment. 


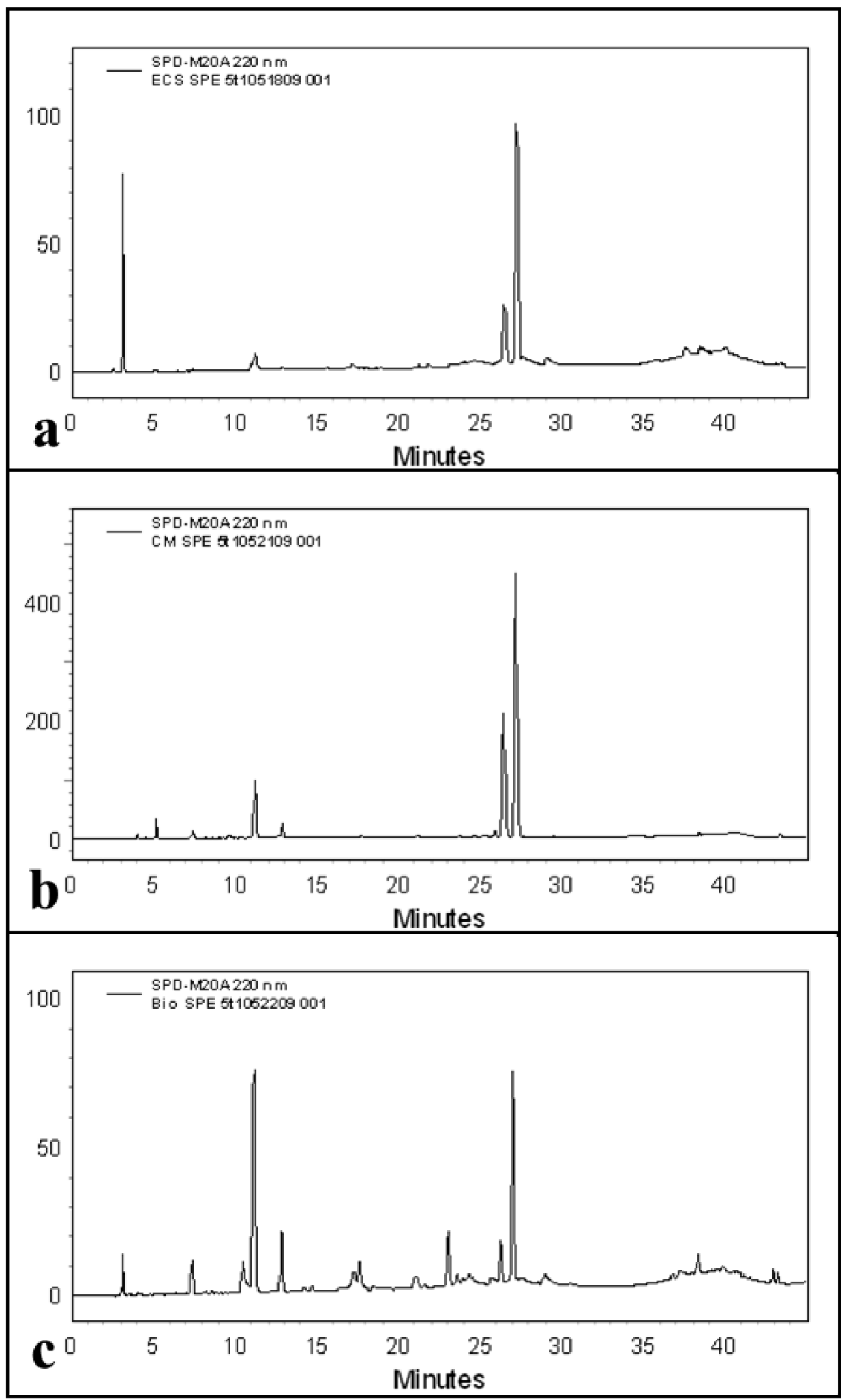

Figure 9: HPLC analysi of different components of Fischerella 52-1; a) ECS, b) Culture Medium, and c) Biomass 


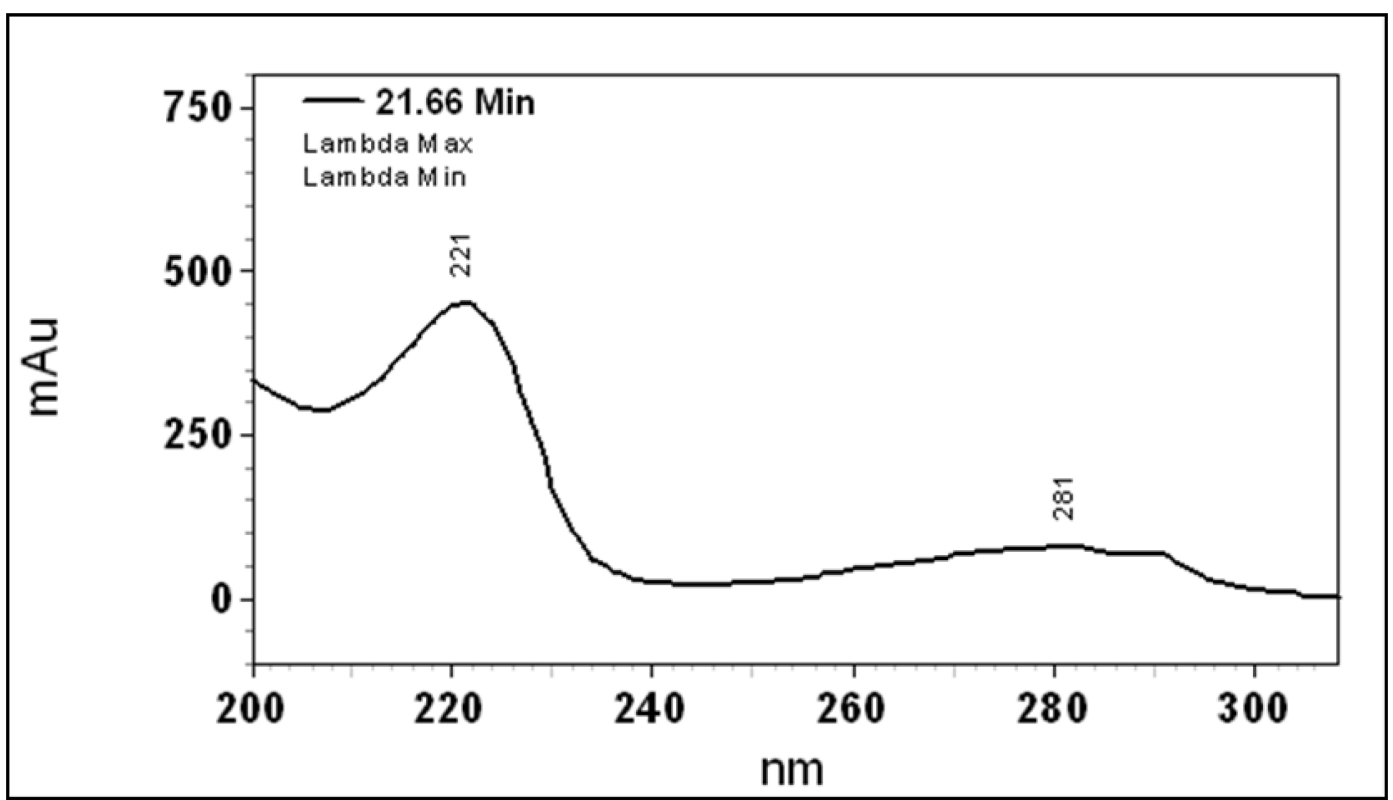

Figure 10: Indole Chromaphore from ECS produced by Fischerella 52-1 obtained from the HPLC

\subsubsection{Isolation of Indole Alkaloids}

The ECS fractionation obtained by silica SPE was scaled up to a silica glass column and the bioactivity was again observed in the fractions from the 5:1 hexane to acetone solution. Subsequently three HPLC methods were used to isolate indole compounds from the Extracellular Solid. The initial method of isolation utilized the same acetonitrile gradient specified in the methods section. The semiprep column was also used in the second method, which utilized an isocratic 55\% acetonitrile concentration for 70 minutes, followed by a 5 minutes flush. The previously determined bioactive fraction, was collected in the time range of 62 to 68 minutes using the second method. The third method, however, used the C18 Kinetex column with an isocratic method of $60 \%$ methanol at a flowrate of $1 \mathrm{ml} / \mathrm{min}$. Unfortunately this method required an oven temperature of $34^{\circ} \mathrm{C}$, and most of the time the pressure of the HPLC pump reached the maximum limit. This purification scheme, furthermore, produced too little of the isolated 
compounds to obtain ${ }^{13} \mathrm{C} \mathrm{NMR}$ data. It was subsequently discovered that the active compounds were only partially soluble in many of the organic solvents used. Further investigation revealed that the active compounds were quite soluble in methanol, and that when the ECS was extracted with methanol, mostly the indole containing compounds were extracted. Using methanol to extract hapalindoles was previously documented by Doan et al., (2000) who were able to extract 12-epi-Hapalindole E Isonitrile from Fischerella using methanol. Since the compound of interest is most likely a hapalindole this would presumably be a better method of extraction. The optimized extraction process with methanol was used for the initial extraction of the ECS with subsequent HPLC methods to obtain isolated indole compounds.

The methanol extract of the ECS was separated using two HPLC methods. In the first HPLC method, which utilized the 50\%-99\% acetonitrile and water gradient, a 5 minute segment was collected from minute 15 to minute 20 in the gradient. This part of the chromatogram was shown to have most of the indole containing compounds and the main indoles in the ECS. The 15-20 minute fraction was tested using the zebrafish embryo assay and was shown to be bioactive. Once the activity was confirmed, the fraction was then separated using the second HPLC method that utilized a flatter methanol gradient. The method resulted in eight peaks with relatively high absorbance at 225nm and a pronounced chromophore that resembled an indole. Figure 11 shows the chromatogram from this method. 


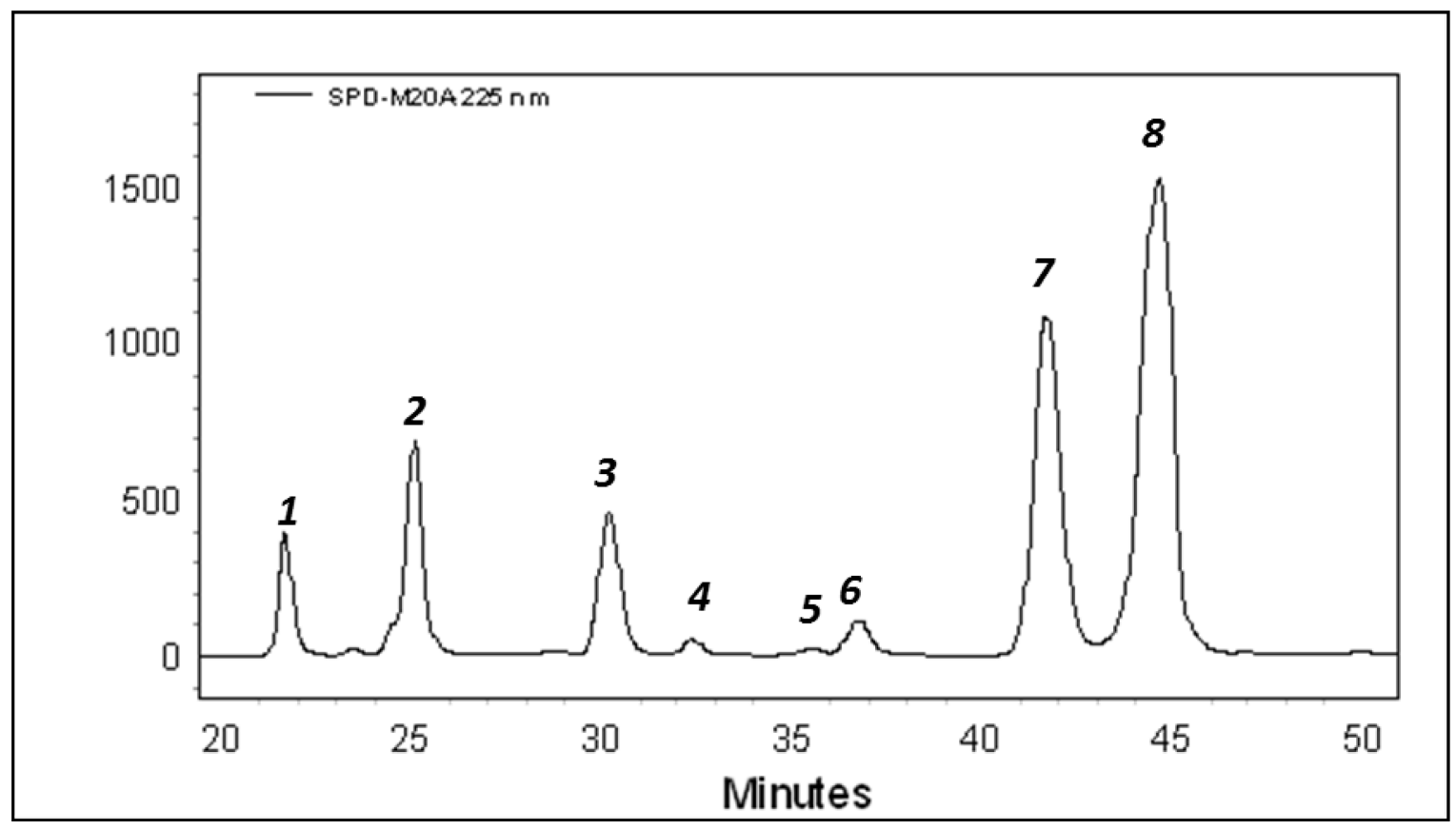

Figure 11:HPLC Method 2 Chromatogram of ECS material

\subsubsection{Chemical and Toxicological Investigation of Main Indole Alkaloids}

The eight peaks observed from the second HPLC method were fractioned into 6 fractions and tested against the zebrafish embryo bioassay. The composition of each fraction and observed bioactivity are given in Table 4. Images of the described bioactivity are given in Figure 12. 
Table 4: Composition of HPLC Fractions and Observed Bioactivity

\begin{tabular}{|l|l|l|}
\hline Region & Included Peaks & Observed Bioactivity at Day 5 \\
\hline 1 & Peak 1 & No specific phenotype observed but different from controls \\
\hline 2 & Peak 2 & No observed difference from controls \\
\hline 3 & Peak 3 & Pericardial edema \\
\hline 4 & Peak 4-6 & No observed difference from controls \\
\hline 5 & Peak 7 & Slight curved body, apparent hump \\
\hline 6 & Peak 8 & "puffy" toxitype \\
\hline
\end{tabular}




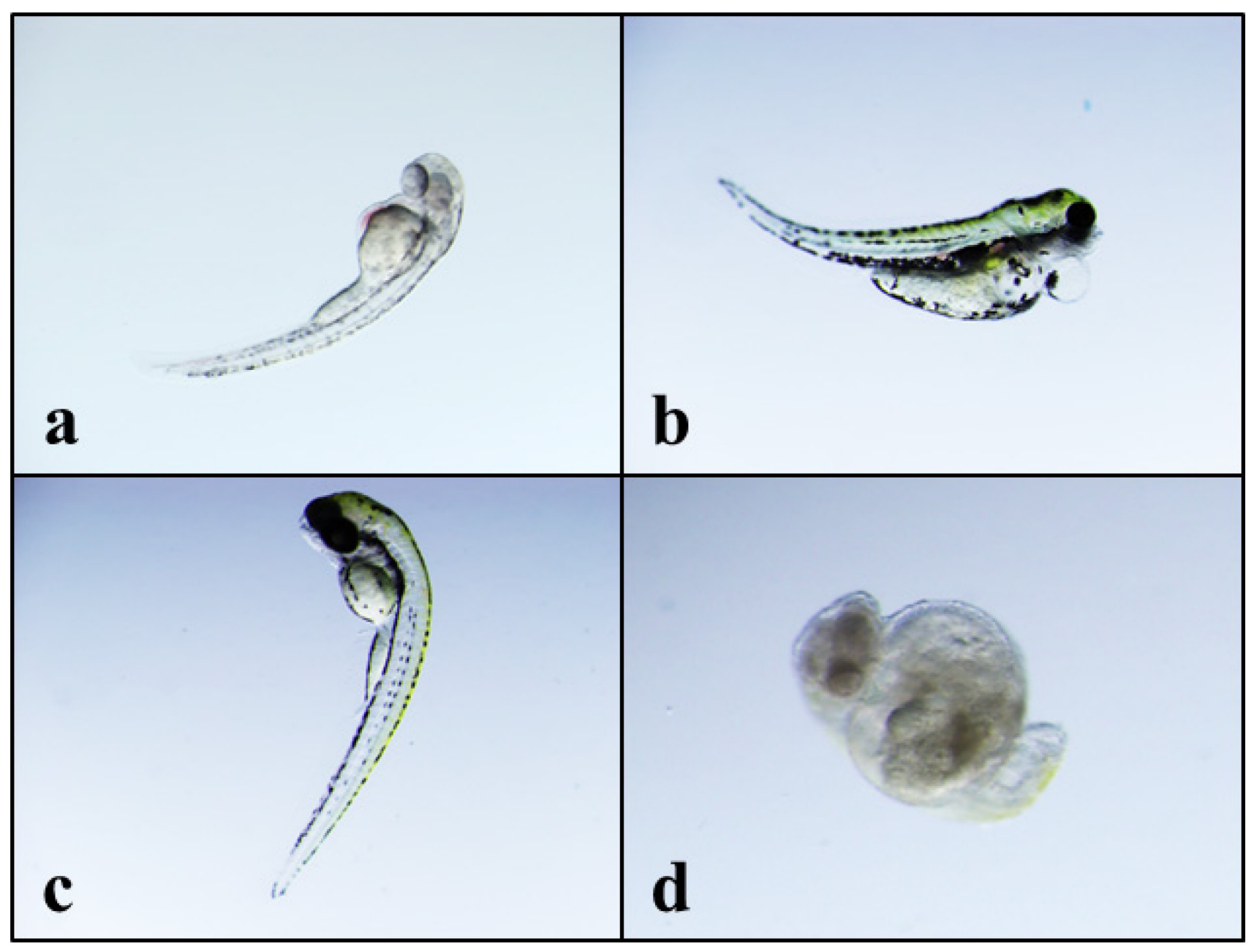

Figure 12: Bioactivity of Active Fractions Observed 5 dpf; a) Fraction 1, b) Fraction 3, c) Fraction 5, and d) Fraction 6 
From the collected fraction three observed toxitypes were observed that affected all of the embryos in that corresponding well. Fraction 3 produced a curved body and pericardial edema, fraction 5 produced a slight humped body axis, and fraction 6 produced the "puffy" toxitype. Fraction 1 produced a mixed set of results that ranged from lightened embryos to curved embryos. Since the bioactivity was not consistent for all of the embryos in the corresponding well this fraction was not specified as a particular toxitype. Because of the lack of bioactivity of Fraction 4, which might be a result of the relative small amounts of peaks 4-6, this fraction was not collected. Peaks 1-3, 7, and 8 were collected and evaluated for purity using a TSQ Access Triple Quadrupole mass spectrometer and the HPLC. The $\mathrm{m} / \mathrm{z}$ of the presumptive molecular ion of each peak is displayed in Table 5 with corresponding retention times and $\lambda_{\max }$.

Table 5: Mass and UV $\lambda_{\max }$ of Peaks 1-3, 7, and 8

\begin{tabular}{|c|c|c|c|}
\hline Peak & Retention Time (min) & $\boldsymbol{\lambda}_{\max }(\mathbf{n m})$ & {$[\mathbf{M}]^{+} \mathbf{m} / \mathbf{z}$} \\
\hline 1 & 21.680 & $221,281,290 \mathrm{sh}$ & 304 \\
\hline 2 & 25.068 & 225,277 & 302 \\
\hline 3 & 30.204 & $223,279,290 \mathrm{sh}$ & 388 \\
\hline 7 & 41.696 & $222,279,290 \mathrm{sh}$ & 422 \\
\hline 8 & 44.648 & $218,278,290 \mathrm{sh}$ & 304 \\
\hline
\end{tabular}

The mass range of Peaks 1 and 8 suggest a hapalindole such as Hapalindole C, H, and J Isonitrile discussed in Chapter 1 (Moore et al., 1987). The difference of two mass units between Peak 2 and 1 and 8 suggest a greater degree of unsaturation. The masses of peaks 3 and 7 are too high for a hapalindole, but fall into the mass range of the ambiguines also discussed in Chapter 1 (Smitka et al., 1992). In fact, the mass of Peak 7 
matches the mass of Ambiguine B Isonitrile and Peak 3 matched Ambiguine C Isonitrile (Smitka et al., 1992). Further chemical characterization of Peaks 7 and 8 is presented in Chapter 3.

Purified samples of Peaks 3, 7, and 8 were tested against the zebrafish model at a range of concentrations $0,0.1,0.5,1,5$, and $10 \mu \mathrm{g} / \mathrm{mL}$. The second part of this assay incorporated the embryo removal experiments from the media containing isolated peaks to determine whether the embryos were able to reverse the developmental defects. In the exposure assay, Peak 7 at a concentration of $10 \mu \mathrm{g} / \mathrm{mL}$ and Peak 8 at a concentration of 5 and $10 \mu \mathrm{g} / \mathrm{mL}$ produced abnormally developed zebrafish embryos. The observations of the assay at 3 dpf are shown in Figure 13. Peak 3 did not show any bioactivity which may be the result of low concentration. 


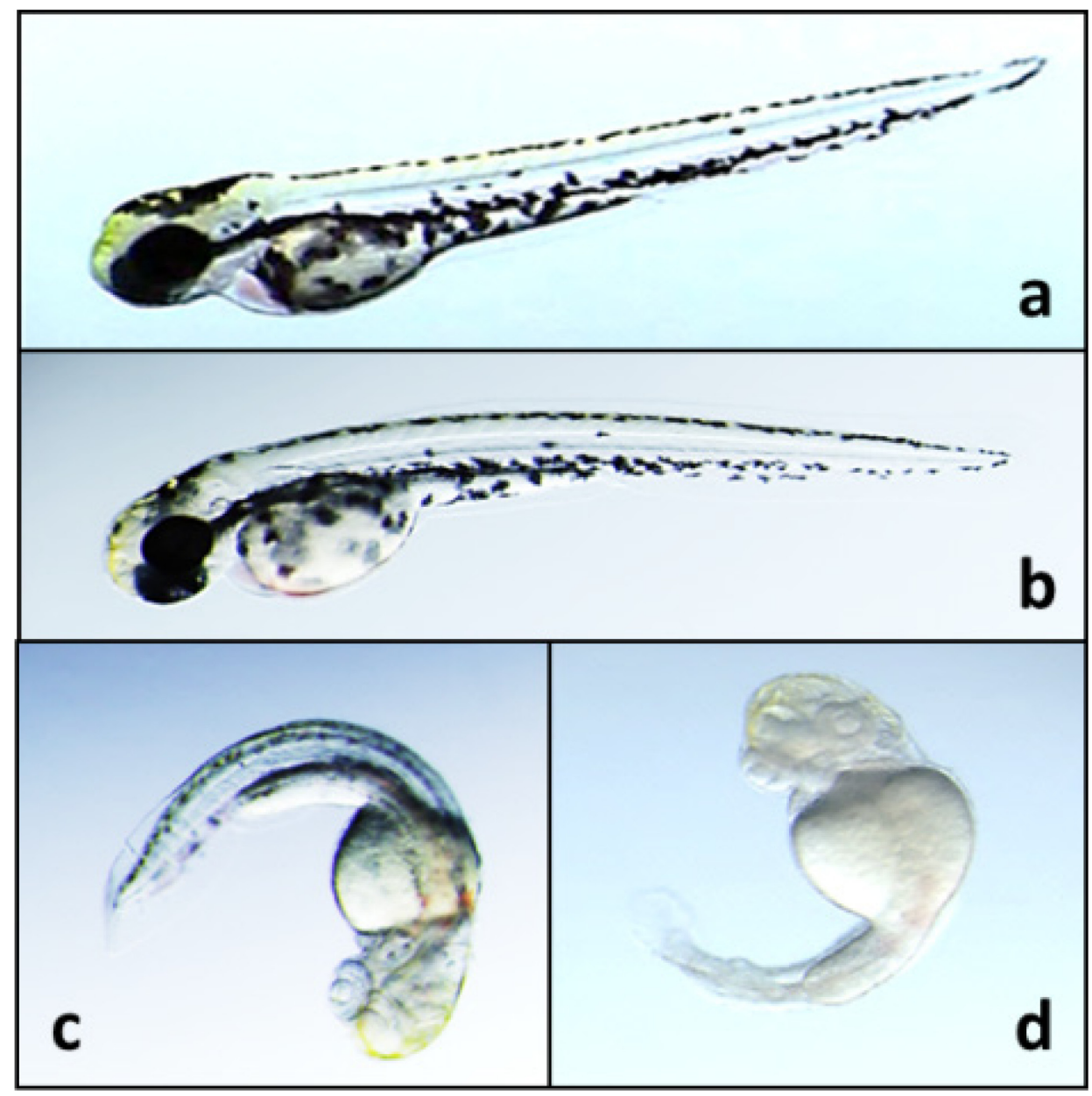

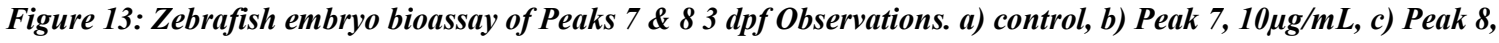
$5 \mu \mathrm{g} / \mathrm{mL}$, and d) $10 \mu \mathrm{g} / \mathrm{mL}$

Compared to the control, the embryos exposed to $10 \mu \mathrm{g} / \mathrm{mL}$ of Peak 7 showed a slight curvature of the body. This defect was eventually overcome at $5 \mathrm{dpf}$ when the embryos hatched, as seen in Figure 14. 


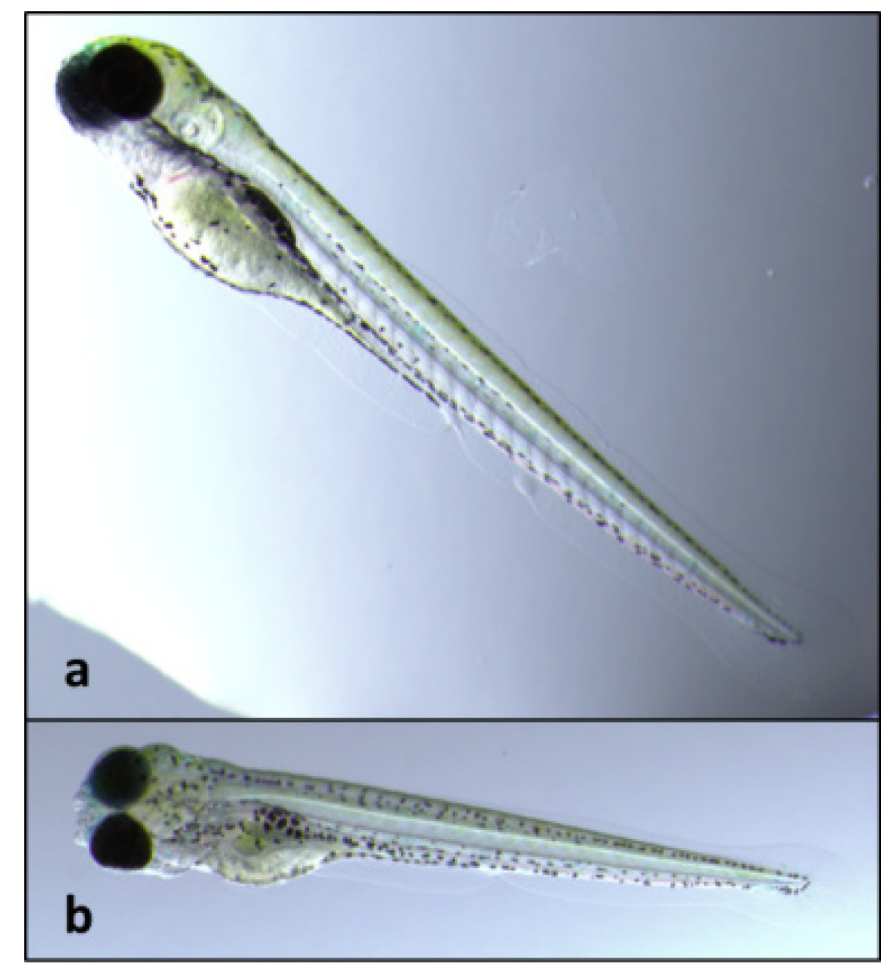

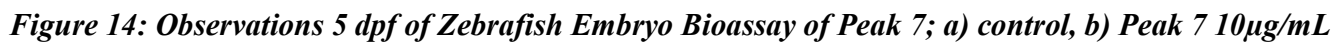

The embryos exposed to Peak 8 at 5 and $10 \mu \mathrm{g} / \mathrm{mL}$ did not hatch at $5 \mathrm{dpf}$, and were severely deformed. The defects observed included a curved body axis, lack of melanophores, and pericardial edema, as depicted in Figure 15. As extensive as these deformities were, it was observed that if the embryos were removed from the chorion and into a fresh E3 medium that some of the effects could be reversed. Figure 16 shows the extent of the developmental deformities throughout the time span of the bioassay, and the extent of the reversal of the deformities in removed embryos at a concentration of Peak 8 at $5 \mu \mathrm{g} / \mathrm{mL}$. It was observed that if the embryo was removed at $2 \mathrm{dpf}$, that at $5 \mathrm{dpf}$ the embryo appeared very similar to the control, the only difference was the retention of a slight pericardial edema. When the embryo was removed at day 3 the edema was more pronounced, but the embryo recovered much of the lost color and the body axis of the embryo was less curved. 


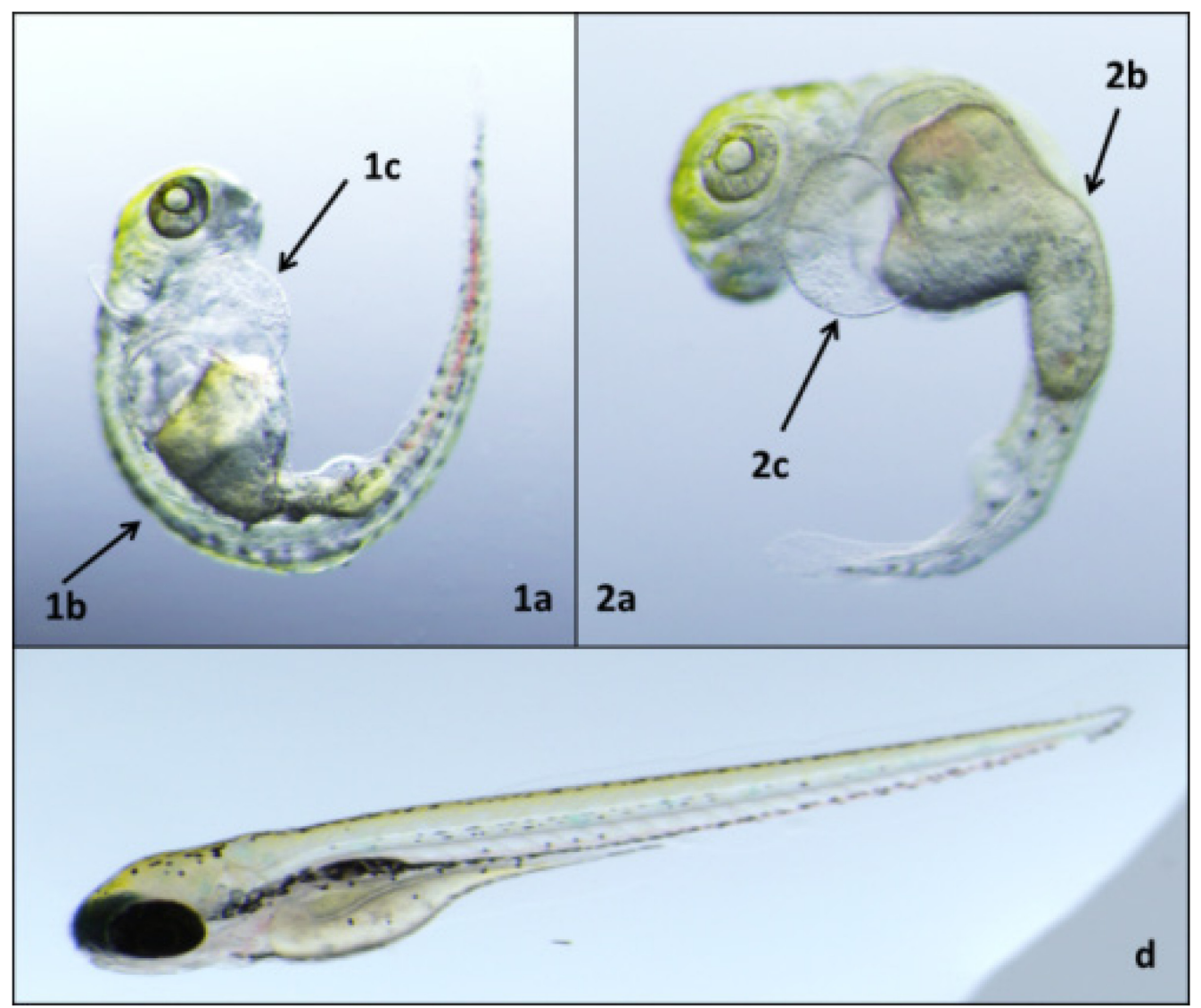

Figure 15: Zebrafish Embryo Assay Peak 8: 1) $5 \mu \mathrm{g} / \mathrm{mL}$ and 2) 10 $\mu \mathrm{g} / \mathrm{mL} 5$ dpf Observations; a) Overall lack of pigment, b) abnormal curvature of the body, c) pericardial edema, d) control 


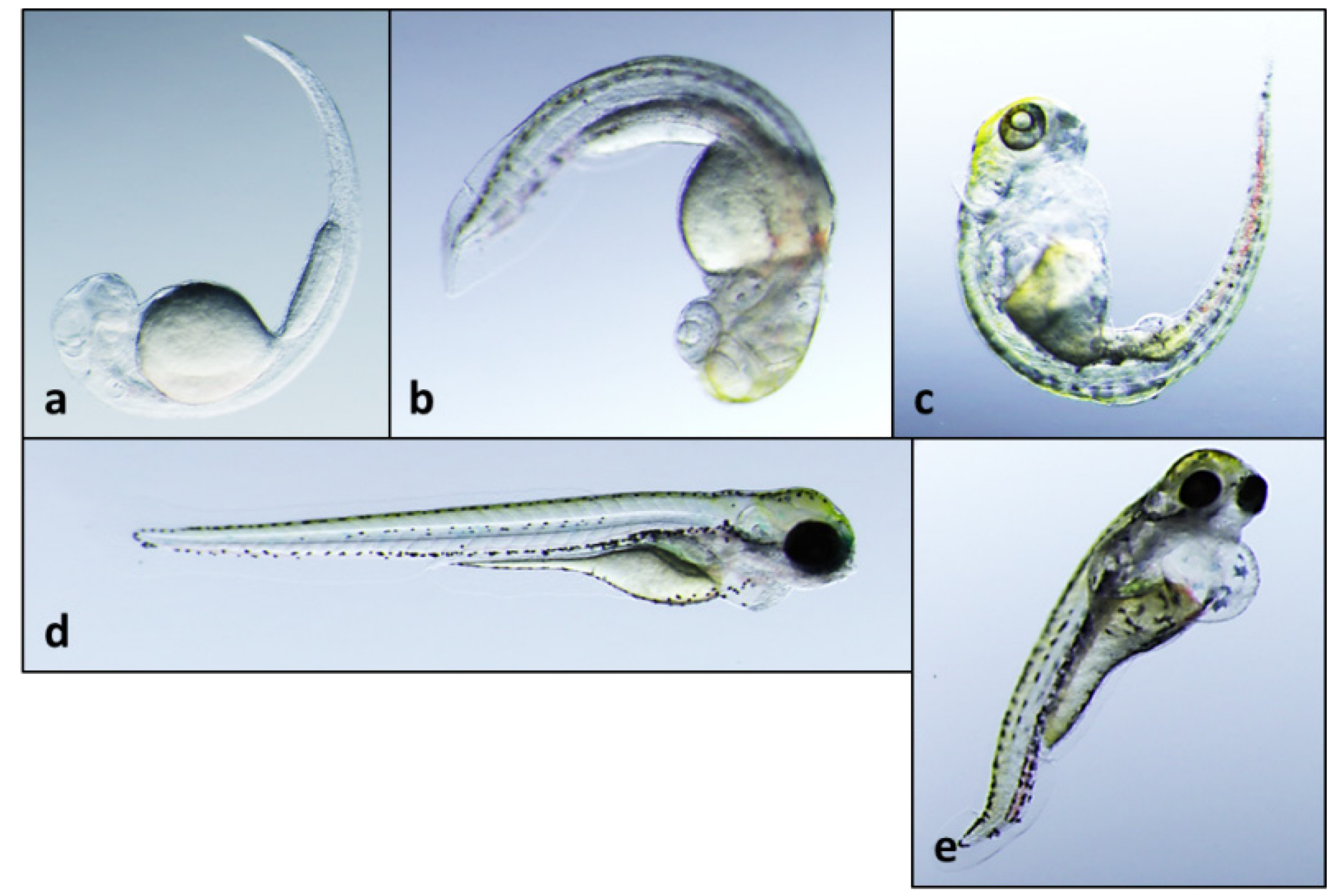

Figure 16: Zebrafish Embryo assay $5 \mu \mathrm{g} / \mathrm{mL}$ Peak 8 Observations; a) 2 dpf, b) 3 dpf, c) 5 dpf, d) Removed 2 dpf Observed 5 dpf, e) Removed 3 dpf Observed 5 dpf 
It is shown in Figure 17 that at a concentration of $10 \mu \mathrm{g} / \mathrm{mL}$ of Peak 8, embryos removed at 2 and 3 dpf into toxin-free E3 medium were not able to reverse the effects of the compound at $5 \mathrm{dpf}$. At this concentration it is observed that the embryos are unable to reverse the edema or the curved body axis. However, if the embryo is removed as early as $2 \mathrm{dpf}$ it appears to begin producing melanin producing cells. 


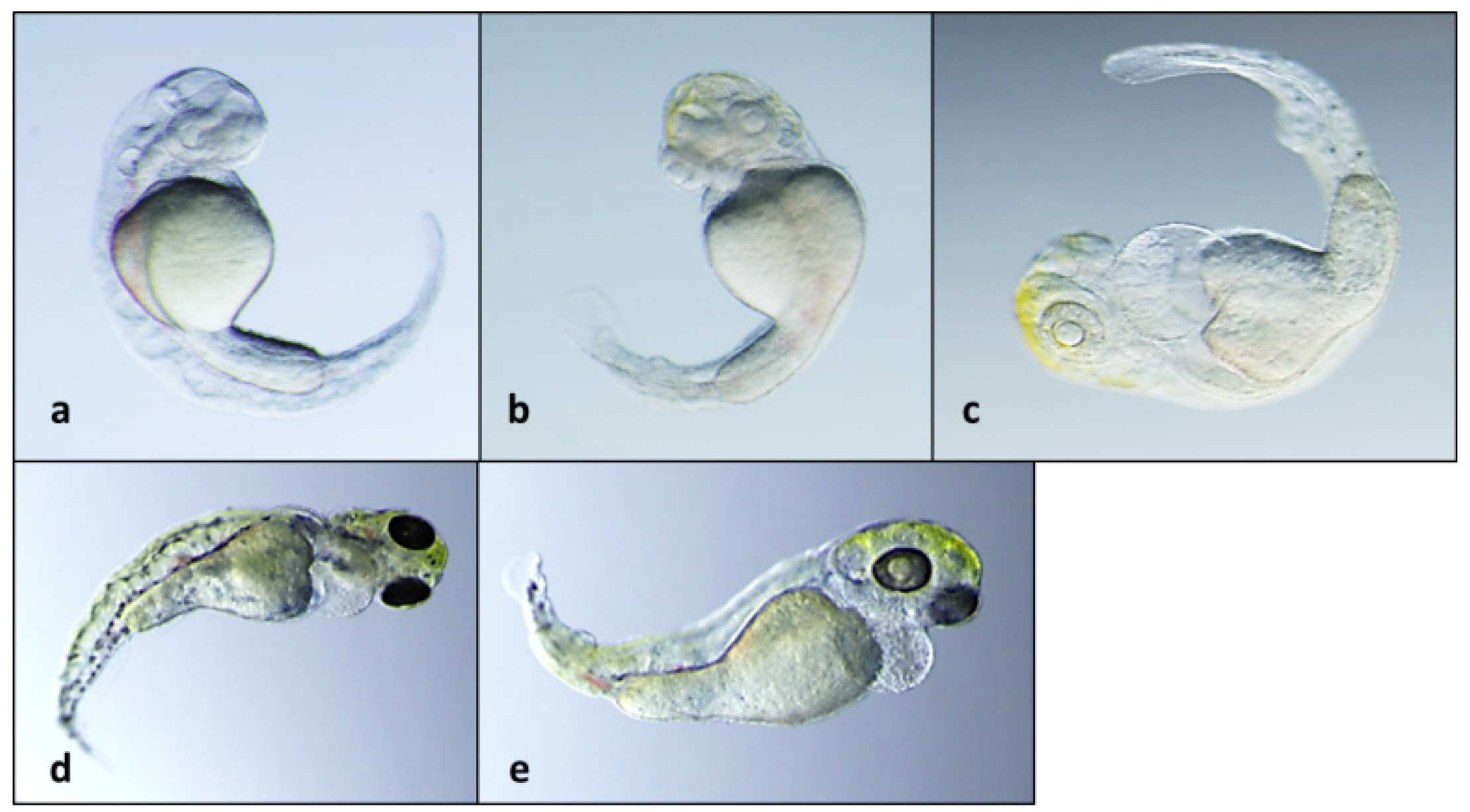

Figure 17: Zebrafish Embryo assay $10 \mu \mathrm{g} / \mathrm{mL}$ Peak 8 Observations; a) 2 dpf, b) 3 dpf, c) 5 dpf, d) Removed 2 dpf Observed 5 dpf, e) Removed 3 dpf Observed 5 dpf 
As a result of the lack of observed bioactivity with Peak 3, at a concentration as high as $10 \mu \mathrm{g} / \mathrm{mL}$, another zebrafish embryo assay was performed. A more concentrated solution of region 3, which includes Peak 3 with small amounts of other indoles, was used, however, the exact concentration was not determined. The bioactivity not observed in the previous assay with $10 \mu \mathrm{g} / \mathrm{mL}$ of pure Peak 3 was observed in this assay as shown in Figure 18. The embryos exposed to concentrated region 3 developed a curved and swollen body, and did not develop melanin. The range of bioactivities of many of these compounds is dependent on concentration. Another assay using Peak 3 purified with the zebrafish embryo assay produced embryos that were not able to hatch at $5 \mathrm{dpf}$. One of the embryos was examined and removed from the chorion (Figure 18). The embryo exhibited a pericardial edema and curved body similar to previous observations of region 3.

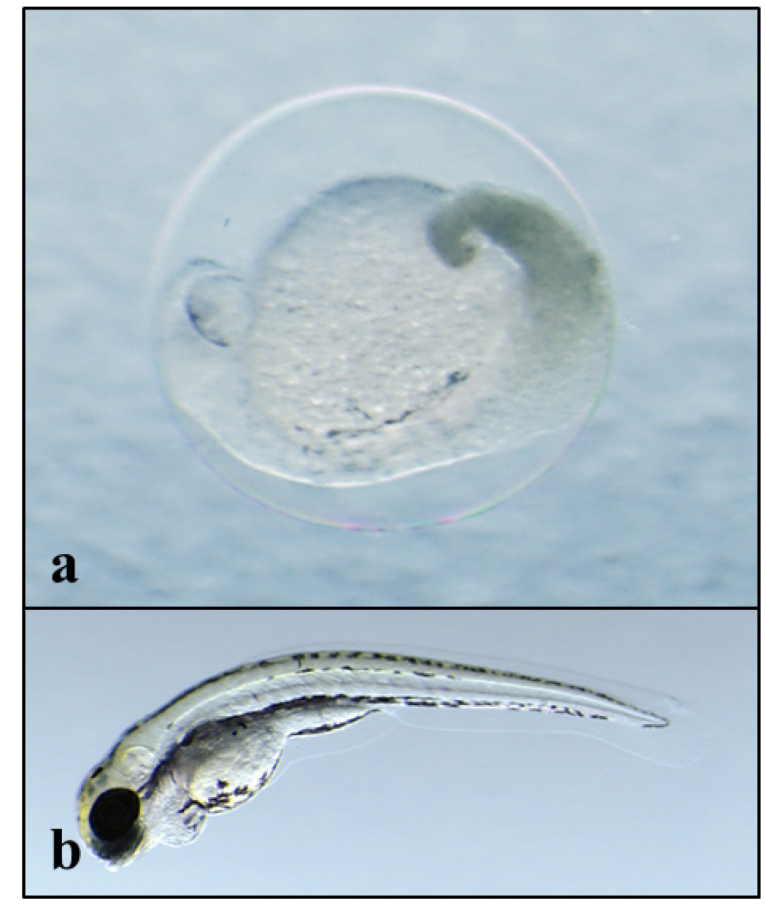

Figure 18: a) Region 3 at 3dpf and b) Purified Peak 3 at 5 dpf 


\subsubsection{Metabolite Solubility Analysis}

The actual amount of each compound that was being exposed to the embryo is uncertain, but mass spectrometric measurements of the same six concentrations of Peak 8 and 7 in E3 showed that very little of these toxins were apparently dissolved in the E3 media. The comparison of the peak areas of the compounds dissolved in methanol, and those dissolved in E3, are shown in Table 6. The methanol blank for the Peak 7 analysis produced an unusually high value for the peak area, this is most likely a result of carryover from the syringe of the autosampler. In order to conserve valuable instrument time, the E3 samples containing Peak 7 were run before the methanol samples which would explain the carryover. It is clear that little of each compound is dissolved in the E3. The low solubility of the bioactive compounds could either indicate the potency of these compounds or elude to an alternative process for the zebrafish embryos to uptake the bioactive material.

Table 6: Mass Spectrometric Analysis of Peak 7 and 8 Solubility

\begin{tabular}{|c|c|c|c|c|}
\hline & \multicolumn{2}{|c|}{ Peak 7 } & \multicolumn{2}{c|}{ Peak 8 } \\
\hline $\begin{array}{c}\text { Concentration } \\
(\boldsymbol{\mu g} / \mathbf{m L})\end{array}$ & Methanol & E3 & Methanol & E3 \\
\hline 0 & 33519 & 0 & 0 & 0 \\
\hline 0.1 & 6452857 & 164840 & 366698 & 0 \\
\hline 0.5 & 40511892 & 943260 & 1364226 & 0 \\
\hline 1 & 76926182 & 1309324 & 2970130 & 0 \\
\hline 5 & 357281234 & 3282137 & 13429613 & 0 \\
\hline 10 & 518430653 & 5228898 & 22780463 & 28670 \\
\hline
\end{tabular}




\subsubsection{Mosquito Larvicidal Bioassay}

The toxicity of the indole fraction of the ECS and the purified Peak 8, was additionally evaluated with the mosquito larvae. Each of these samples was tested using an non-quantified concentration of ECS and Peak 8, to determine whether any larvicidal activity occurred. The development of the mosquito larvae can be organized into five stages. The first week of development contains the four instar stages which are shown in

\section{Figure 19.}

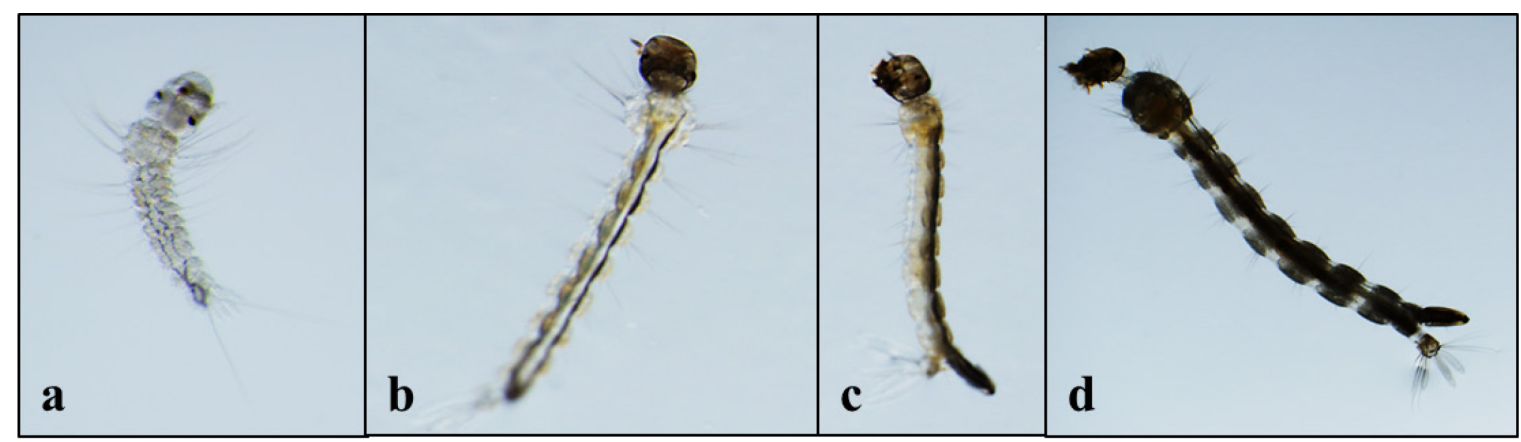

Figure 19: Development of Mosquito Lavae, images are not set to proper size ratio; a) $1^{\text {st }}$ Instar, b) $2^{\text {nd }}$ Instar, c) $3^{\text {rd }}$ Instar, and d) $4^{\text {th }}$ Instar. (Pictures provided by Gerald Berry)

The ECS fraction produced $100 \%$ mortality in 4 days. Delayed development was initially observed since none of the four larvae ever reached the $2^{\text {nd }}$ instar of development which should have occurred at day 2 . At day $1,0 \%$ mortality was observed, but from day 2 to day 4 , a $25 \%$ increase in mortality was observed each day. Even though development was delayed in the first 2 days, no apparent deformities were observed. In order to determine whether the extract was still active after $100 \%$ mortality was confirmed at day 4, four more larvae were introduced into the well containing the ECS indole fraction. At day 1, no activity was observed, and the larvae were at the $1^{\text {st }}$ instar. However, at day $2100 \%$ mortality was observed. 
Since mass spectrometry supported Peak 8 to be a hapalindole, and that some hapalindoles have been shown to be larvicidal, it was evaluated using the mosquito larvicidal assay (Becher et al., 2007). Peak 8 produced a different bioactivity compared to the ECS indole fraction. The concentration of the purified Peak 8 was not measured for the assay. No mortality was observed for the first two days of the assay, but on the second day, the larvae's movement decreased, as observed using light microscopy and compared to the negative controls. Also, the development of the larvae was delayed, and all of the larvae specifically remained at the $1^{\text {st }}$ instar stage. On day $4,25 \%$ mortality was observed, and all of the embryos were still at the $1^{\text {st }}$ instar stage of development. At day $5,50 \%$ mortality was observed, and one of the larvae was shown to be at the $2^{\text {nd }}$ instar stage, while the remaining larva was still at the $1^{\text {st }}$ instar. The $2^{\text {nd }}$ instar larva showed some defects in its development, which was apparent by comparing to a $2^{\text {nd }}$ instar control in Figure 20. The control showed a deeper color in the head region compared to the larva exposed to Peak 8. The same reduced color was observed in the body axis of the larva exposed to Peak 8, compared to the control. Additionally an abnormal brown material was observed in the larva exposed to the Peak 8 which was not observed with the control. Considering the color of the liver powder the larvae were fed was similar to the material observed, the digestion of the liver powder might have been impeded, which was subsequently accumulated in the gut of the larva. 


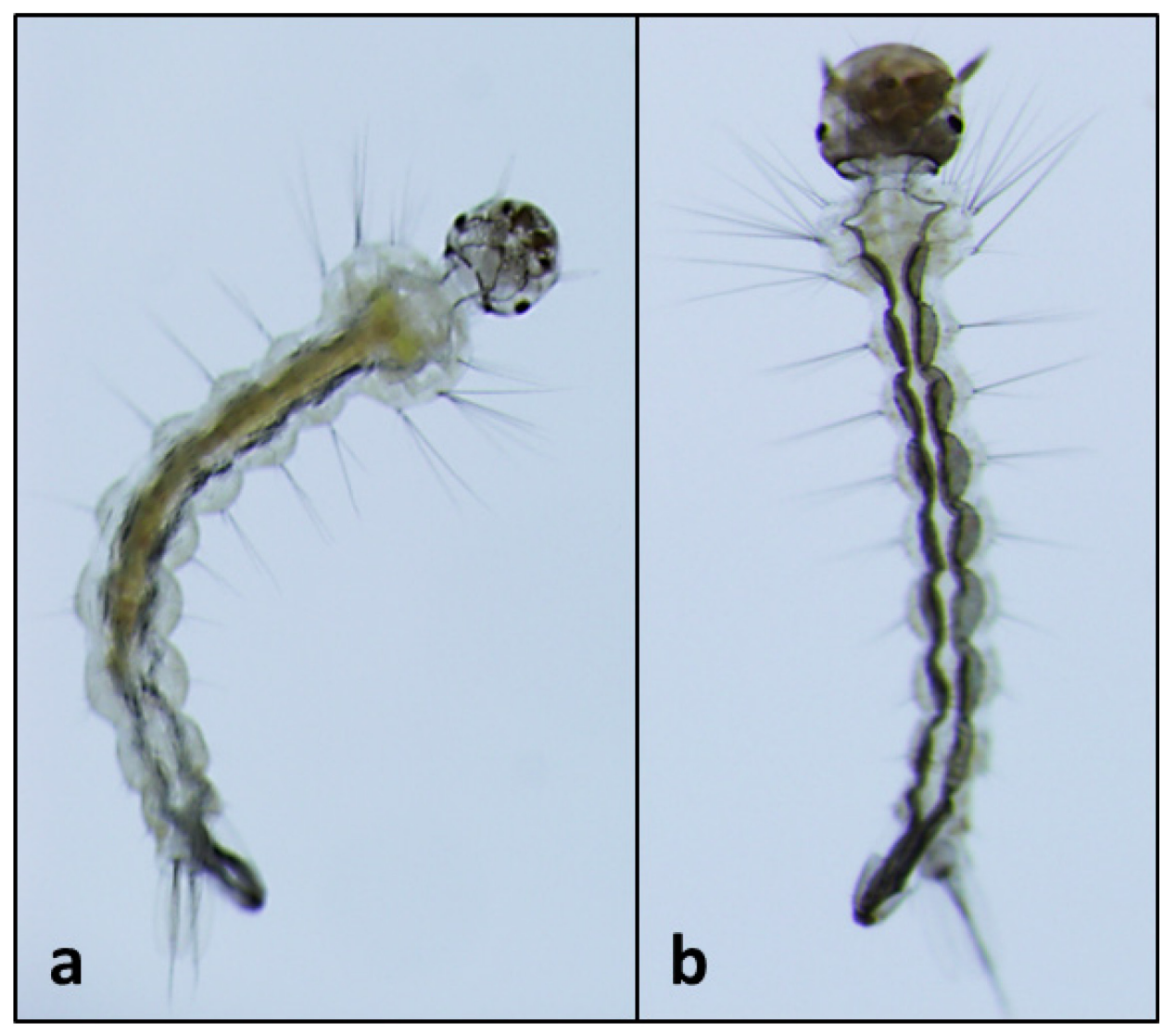

Figure 20: Mosquito Larvacidal Assay Peak 8, a) Peak 8 Day 6 and b) Control 2nd Instar(Pictures produced by Gerald Berry) 


\subsection{Conclusion}

The zebrafish embryo assay was used to investigate the toxicological properties of crude extracts of the Fischerella 52-1 biomass, culture medium, and ECS, and isolated peaks from the ECS. The assay was also used to guide the fractionation of peaks with indole chromophores from the ECS. Of the peaks evaluated with the zebrafish embryos, Peak 8 was observed to produce a higher level of bioactivity compared to Peak 7 . Peak 3 was also evaluated with the zebrafish embryo assay but the purified material did not produce any observable activity at concentrations as high as $10 \mu \mathrm{g} / \mathrm{mL}$. A second analysis with a larger amount not quantified was exposed to the zebrafish embryos and then produced observable defects. The evaluation of these peaks using the zebrafish embryo assay revealed various types and degrees of bioactivities.

The contribution of each individual bioactive peak to the "puffy" toxitype was not determined in the previously discussed experiments, but the toxicological effects of each peak could be compared to the crude ECS extract. Peak 8 was shown to produce a unique toxitype that was similar to the "puffy" toxitype produce by embryos exposed to the crude ECS extract. Peak 8 produced a curved body axis, pericardial edema, and bleached effect in the exposed embryos (Figure 15). The bioactivity produced by Peak 8 was similar to the embryos exposed to the crude material where the embryos were lacking in color and developed no discernible body axis (Figure 8). Peak 7 produced only a slight humped body axis in the zebrafish embryos. Peak 7 may contribute to the overall "puffy" toxitype through a synergistic effect with the other bioactive peaks. Region 3 produced a toxitype that very closely resembled the "puffy" toxitype, but the 
isolated Peak 3 showed a lesser degree of bioactivity. Unfortunately since the concentration of region 3 and Peak 3 were not quantified, it is possible that only a low amount of Peak 3 was exposed to the zebrafish compared to the concentration found in the region 3 exposure. The difference in concentration would explain the low bioactivity. Analysis of the extracts of the culture medium and the biomass, using the LCMS, showed that both Peak 3 and Peak 7 were able to be detected in the other components of the Fischerella 52-1 culture including the extracted biomass and culture medium (Figure 21 and Figure 22). The analysis of other parts of the culture shows that these indole alkaloids are ubiquitous in the culture. The analyses suggest that the indole alkaloids are formed in the cells and exuded into the surrounding environment. In order to reveal a possible reason for the activity observed by Peak 8 , and the lack of strong activity by Peak 7, these compounds were chemically characterized by mass spectrometry and nuclear magnetic resonance spectroscopy. The characterization data is presented and analyzed in Chapter 3. 


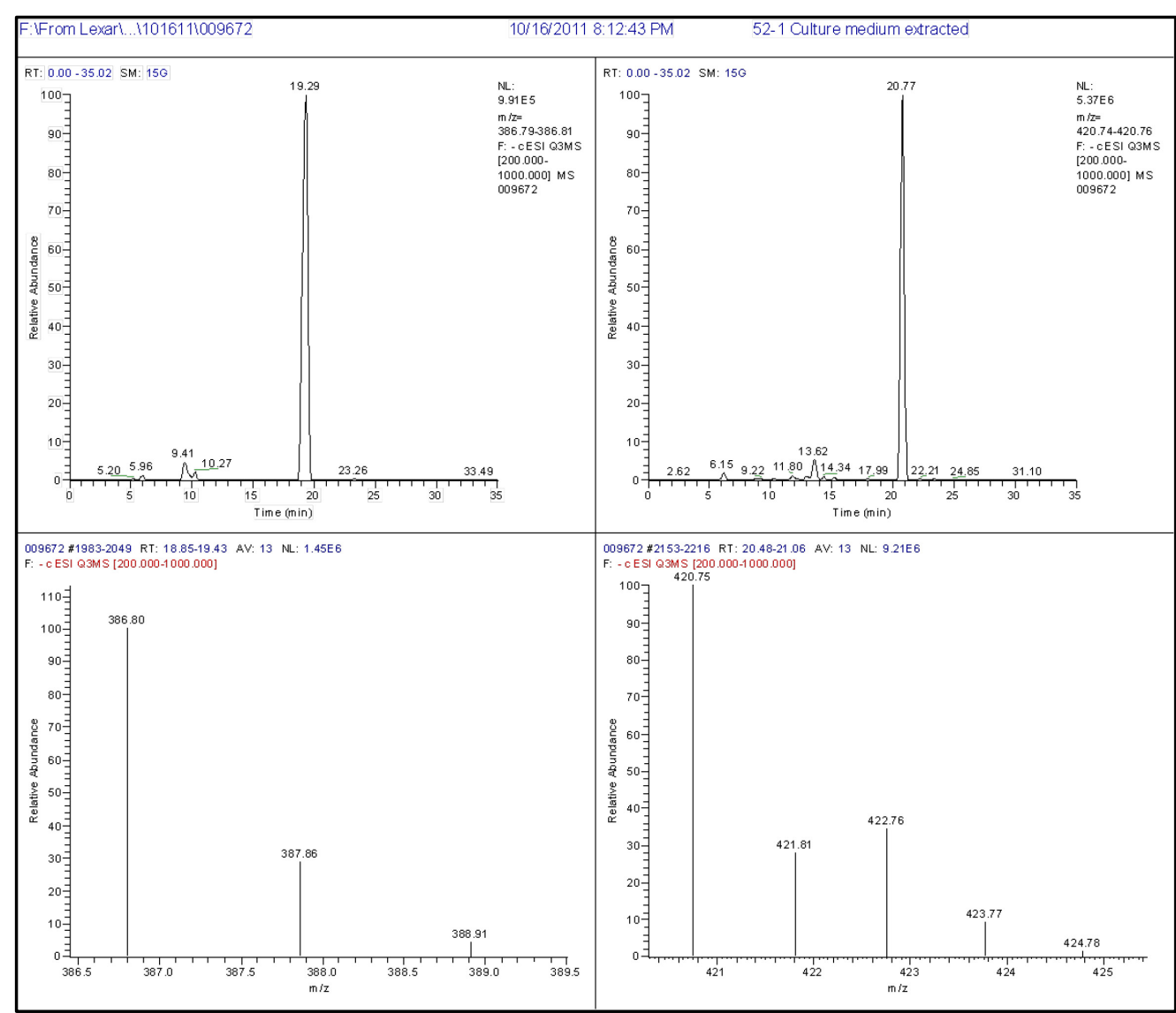

Figure 21: Peak 3 and 7 identified in the Culture Medium by LCMS 


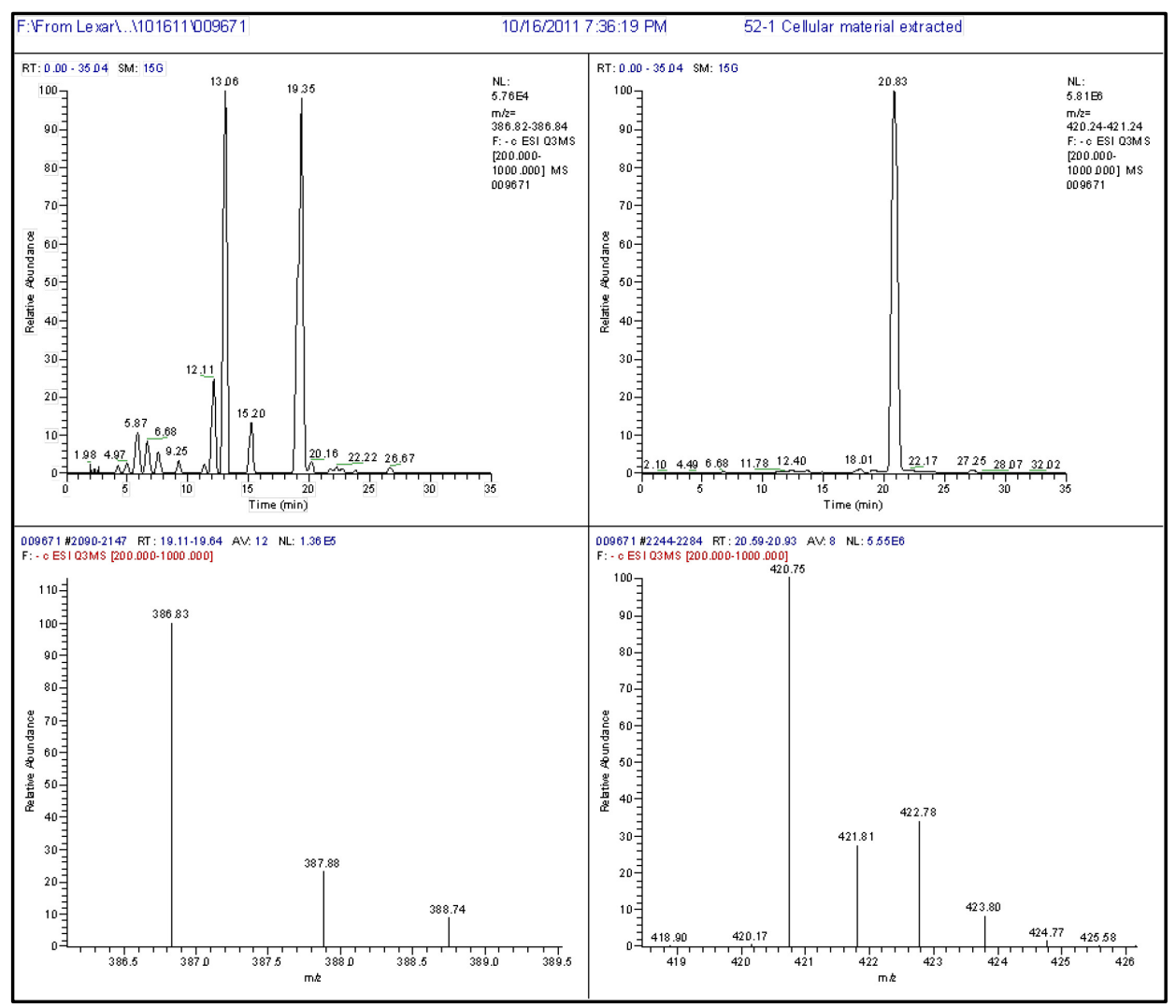

Figure 22: Peak 3 and 7 identified in the Biomass Extract by LCMS 


\section{CHEMICAL CHARACTERIZATION OF MAJOR INDOLE ALKALOIDS FROM FISCHERELLA 52-1}

\subsection{Introduction}

Fischerella has been previously reported to produce various indole alkaloids. From the bioactive fraction analyzed in Chapter 2, eight peaks were observed with indole chromophores. Three peaks were collected and evaluated for purity using the TSQ Quantum Access Mass Spectrometer. From Peaks 7 and 8, two compounds were purified, which included the previously isolated compound 12-epi-Hapalindole $\mathrm{H}$ Isonitrile (1) and the novel compound 12-epi-Ambiguine B Nitrile (2). The structure of these compounds was deduced using various identification techniques including Nuclear Magnetic Resonance Spectroscopy, Mass Spectrometry, and Infrared Spectroscopy (Figure 23).

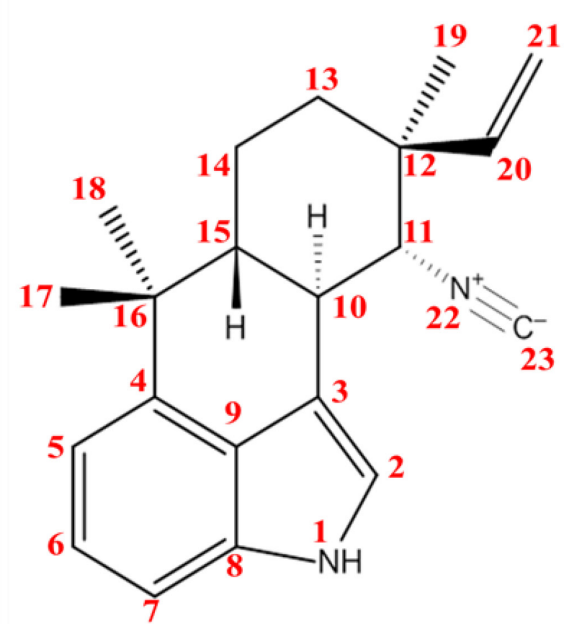

12-epi-Hapalindole $\mathrm{H}$ Isonitrile (1)

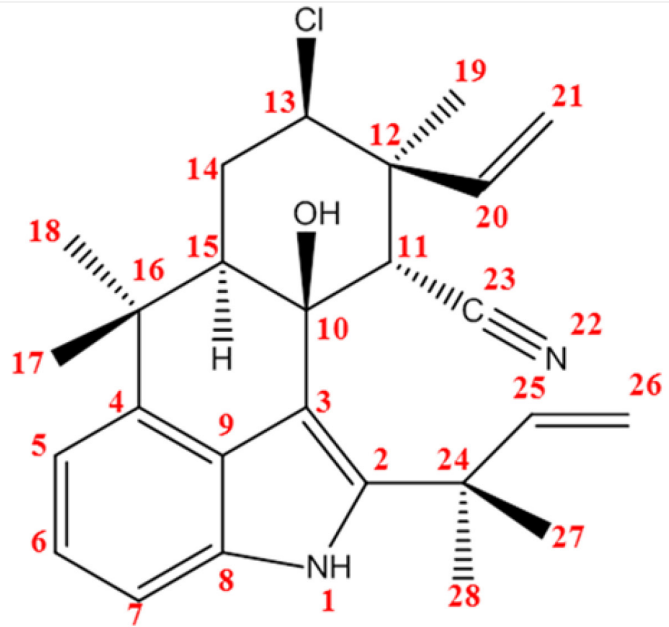

12-epi-Ambiguine B Nitrile

(2)

Figure 23: Structures of 12-epi-Hapalindole H Isonitrile (1) and 12-epi-Ambiguine B Nitrile (2) (Klein et al., 1995 and Smitka et al., 1992) 
The compound 12-epi-Hapalindole H Isonitrile (1), as discussed in Chapter 1, was previously isolated from Hapalosiphon laingii by Klein et al., (1995). The toxicological effects of 1 were not evaluated in this paper, rather this compound was isolated from a purified fraction that was shown to be toxic towards Artemia. The stereoisomer of 1,12epi-Hapalindole J Isonitrile, was shown to be larvicidal for blowfly larvae (Becher et al., 2007). From the toxicological investigation in Chapter 2, 12-epi-Hapalindole H Isonitrile was observed to cause physiological abnormalities in the mosquito larvae which thereby caused larval mortality. In the study be Becher et a., (2007) four isonitrile containing hapalindoles with inverted $\mathrm{C} 12$ stereochemistry were shown to produce larvicidal bioactivities. Which of the two characteristics, the $\mathrm{C} 12$ epimer or the isonitrile group, caused the insecticidal activity has not been determined and deserves further investigation.

The novel compound $\mathbf{2}$ was originally identified as Ambiguine B Isonitrile from the mass spectrometer data, however, further investigation via IR and NMR showed the absence of the isonitrile group (Smitka et al., 1992). It was then observed that the structure for 2 and Ambiguine B Isonitrile were similar, and using various NMR correlation experiments, the structure of $\mathbf{2}$ was deduced. The bioactivity of $\mathbf{2}$ was also investigated in Chapter 2, but was shown to only slightly affect the zebrafish embryos. Compound 2 was not exposed to the mosquito larvae because of the limited quantities available and the large amount needed for the NMR experiments. Further investigation against different organisms will be done in a later study. 


\subsection{Results and Discussion}

\subsubsection{2-epi-Hapalindole H Isonitrile}

One compound was shown to occur in Peak 8, which was purified as an amorphous white solid. The indole moiety structure of $\mathbf{1}$ was originally suggested by the occurrence of the $\lambda \max$ at 221,281 , and 290sh from the HPLC PDA detector. Initial analysis to determine the purity of $\mathbf{1}$ using LCMS produced a peak at 21.86 minutes and was composed of five ion clusters. The three main clusters included $\mathrm{m} / \mathrm{z}$ values of 305 , 278 , and 609 , which were subsequently assigned as $[\mathrm{M}+\mathrm{H}]^{+},[\mathrm{M}-\mathrm{HCN}]^{+}$, and $[2 \mathrm{M}+\mathrm{H}]^{+}$ (Figure 24). A dimer, similar to the dimer observed in the TSQ data, was observed in the ESI analysis of 12-epi-Hapalindole E Isonitrile by Doan et al., (2000). In the Doan et al., (2000) paper the ions observed in the MS analysis included, an ion with $\mathrm{m} / \mathrm{z}$ values of 305 assigned $[\mathrm{M}+\mathrm{H}]^{+}, 327$ assigned $[\mathrm{M}+\mathrm{Na}]^{+}$, and 631 assigned $[2 \mathrm{M}+\mathrm{Na}]^{+}$. Given that dimers were shown to occur in the mass spectrometric analysis of hapalindoles, the ion at 305 was assigned as the hydrogen adduct of the molecular ion. This $\mathrm{m} / \mathrm{z}$ value correlated to the mass range of many of the indole alkaloids previously isolated from Fischerella.

The original extraction method which used hexane and acetone did not afford much of Peak 8, and other compounds in active indole fraction were shown to produce similar molecular ions. The high resolution mass spectrometric analysis of the active indole fraction produced one ion with an $\mathrm{m} / \mathrm{z}$ of 305 that was shown to elute after Peak 7 . The ion with $\mathrm{m} / \mathrm{z}$ of 305.24534 was suggested to be the hydrogen adduct of $\mathbf{1}$. This mass would give the formula $\mathrm{C}_{21} \mathrm{H}_{25} \mathrm{~N}_{2}$, which would then give the molecular formula of $\mathrm{C}_{21} \mathrm{H}_{24} \mathrm{~N}_{2}$ for 1 . The NMR and IR analysis were used to support the proposed formula $\mathrm{C}_{21} \mathrm{H}_{24} \mathrm{~N}_{2}$. 


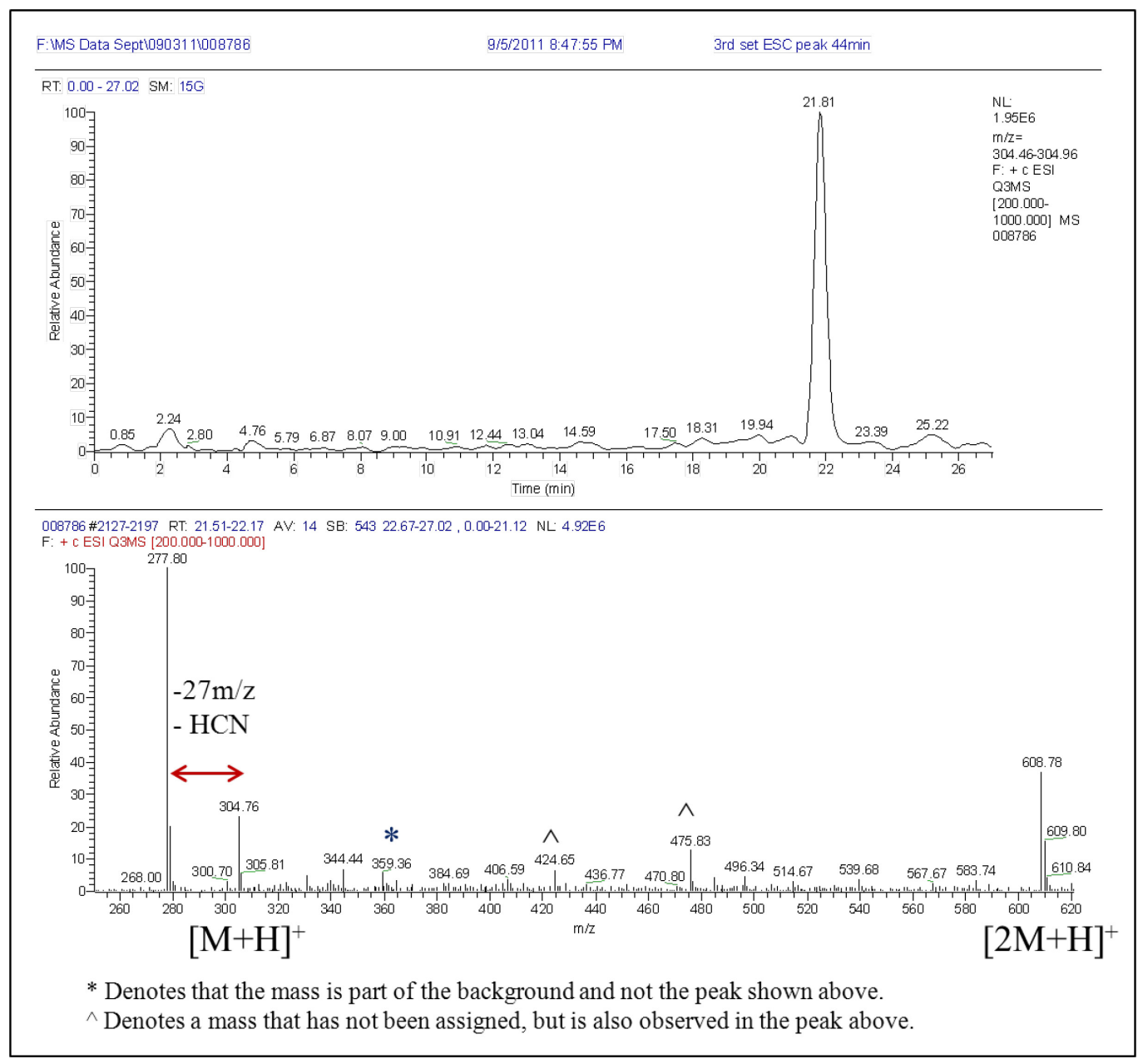

Figure 24: Mass Spectral Analysis of Peak 8

The IR analysis of $\mathbf{1}$ afforded two unique peaks at 3412.10 and $2137.07 \mathrm{~cm}-1$ (Figure 25). The peak at $3412.1 \mathrm{~cm}^{-1}$ was indicative of a primary amine and the peak at $2137 \mathrm{~cm}^{-1}$ was determined to be produced by the isonitrile groups in many of the indole alkaloids produced by Stigonemataceae (Moore et al., 1987 and Pavia et al., 2009). 


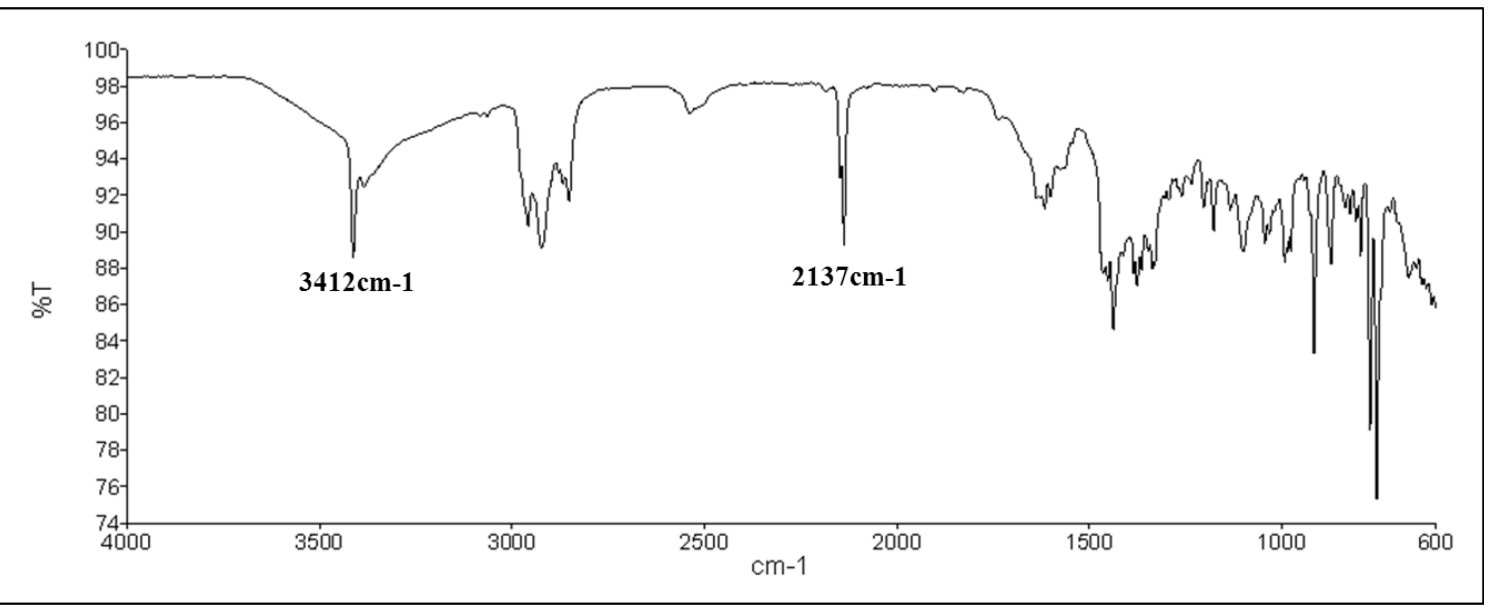

Figure 25: IR of Compound 1

After three consecutive sets of purification from the ECS using the optimized method, sufficient quantities of $\mathbf{1}$ were obtained to allow for carbon NMR experiments to be performed. The NMR experiments performed on 1 included ${ }^{1} \mathrm{H}$ NMR, ${ }^{13} \mathrm{C}$ NMR, COSY, and HMQC and are shown in the following five figures (Figures 26-30). Different solvents were tested to determine the best solvent to improve dissolution and provide chemical shifts without solvent overlapping. From these tests it was determined that $d$-benzene $\left(\mathrm{C}_{6} \mathrm{D}_{6}\right)$ and $d$-methanol (MeOD) were the best solvents and were used to perform two proton NMR experiments. The first solvent was a mixture of $\mathrm{D}_{2} \mathrm{O}$ and $\mathrm{MeOD}$, and the second solvent was $\mathrm{C}_{6} \mathrm{D}_{6}$. The spectrum obtained with the methanol and water mixture provided better resolution in the range of $1-2 \mathrm{ppm}$ range, which allows a number of hydrogens to be distinguished because of less overlapping. In contrast, the spectrum obtained using $\mathrm{C}_{6} \mathrm{D}_{6}$ showed a considerable signal overlap in the 1-2ppm range. Homonuclear Correlation Spectroscopy (COSY) was also performed using both solvent systems, and the same correlations were observed in each. The $\mathrm{C}_{6} \mathrm{D}_{6}$ COSY provided a 
spectrum that was clear of chemical shifts previously thought to be impurities observed in the MeOD spectrum, $\mathrm{C}_{6} \mathrm{D}_{6}$ was primarily used for the COSY experiments.

The ${ }^{1} \mathrm{H}$ NMR of 1 supported the formula of $\mathrm{C}_{21} \mathrm{H}_{24} \mathrm{~N}_{2}$ proposed from the OT Velos data, with 24 protons observed. In the carbon NMR only 19 carbons were observed, the HMQC scan revealed an additional carbon signal not observed in the carbon NMR. With the addition of the carbon from the HMQC one carbon would still be missing thereby disagreeing with the formula. The chemical shift that would have been produced by the carbon in the isonitrile group observed with the IR was not shown in the ${ }^{13} \mathrm{C}$ NMR. The absence of the isonitrile carbon signal has been observed by Becher et al., (2007) with the compound 12-epi-Hapalindole $\mathrm{J}$ Isonitrile when the analysis was performed in $d$-chloroform. Using the IR as evidence for the occurrence of the isonitrile group and the carbons and hydrogens observed in the NMR data the molecular formula would be the same as that proposed from the ion selected in the high resolution mass spectrometric analysis. Using all of the analyses previously discussed, 1 was suggested to have an indole moiety, an isonitrile group, and the formula $\mathrm{C}_{21} \mathrm{H}_{24} \mathrm{~N}_{2}$. The formula is the same as some previously isolated indole alkaloids including Hapalindole H Isonitrile, Hapalindole J Isonitrile, and 12-epi-Fischerindole U Isonitrile (Moore et al., 1987; Stratmann et al., 1994). In order to differentiate between the different families of indole alkaloids, discussed in Chapter 1, the splitting patterns in the aromatic region were analyzed. It was determined that $\mathbf{1}$ was not a fischerindole because of the observed coupling of chemical shifts in the aromatic region of the ${ }^{1} \mathrm{H}$ NMR, which specified the bonding of the indole moiety at the $\mathrm{C} 3$ and $\mathrm{C} 4$ positions. This region also eliminated the ambiguine family by showing a singlet produced by the hydrogen on $\mathrm{C} 2$ in the indole 
moiety. Given that all of the indolinone alkaloids would not produce the observed UV spectrum, and the bonding at the aromatic region of the ${ }^{1} \mathrm{H}$ NMR, the compound was determined to be a hapalindole. 


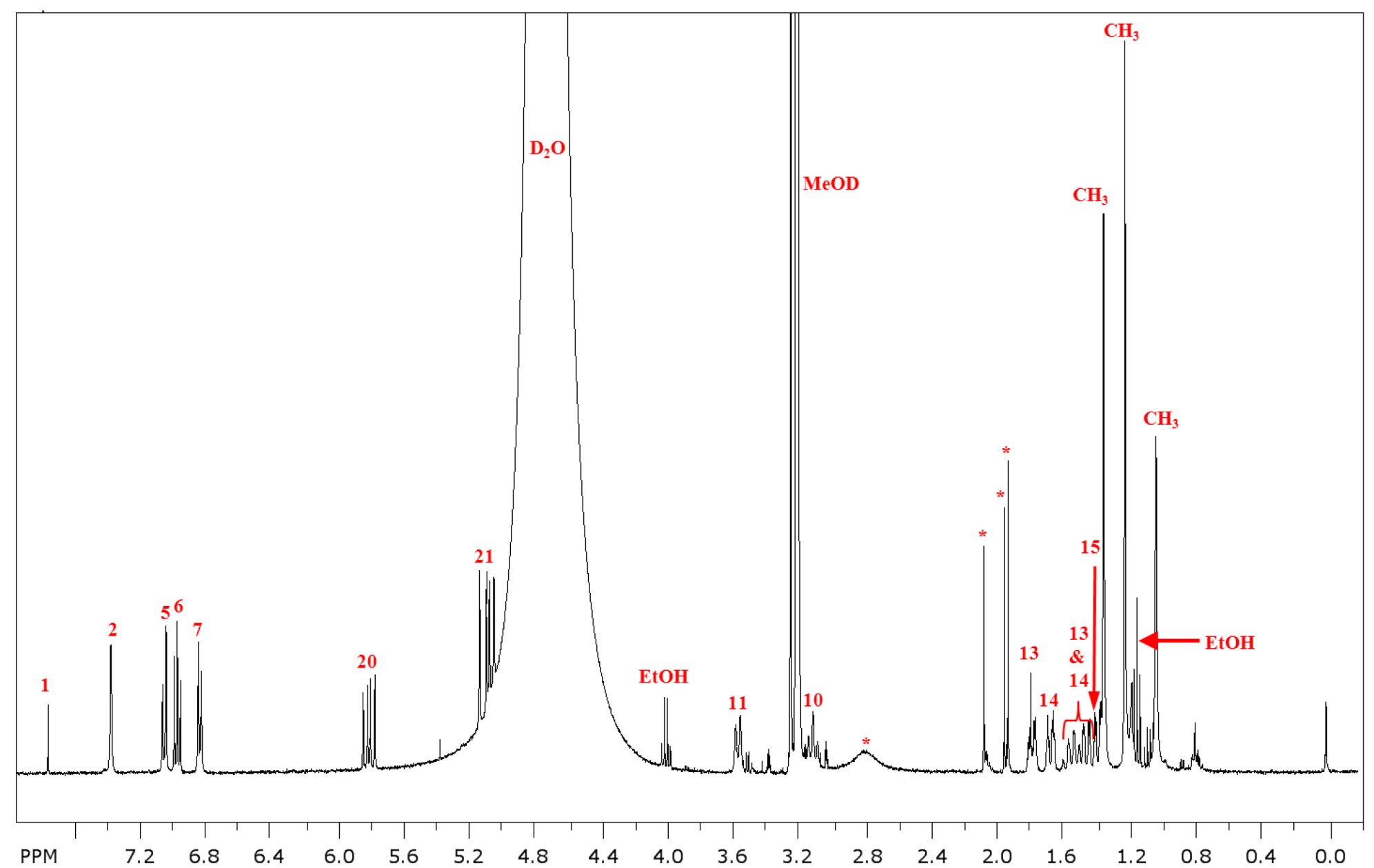

Figure $26{ }^{1} \mathrm{H} N M R$ in $\mathrm{D}_{2} \mathrm{O}$ and MeOD (* Denotes chemical shifts not assigned) The ethanol assigned above was used to clean the NMR tubes. 


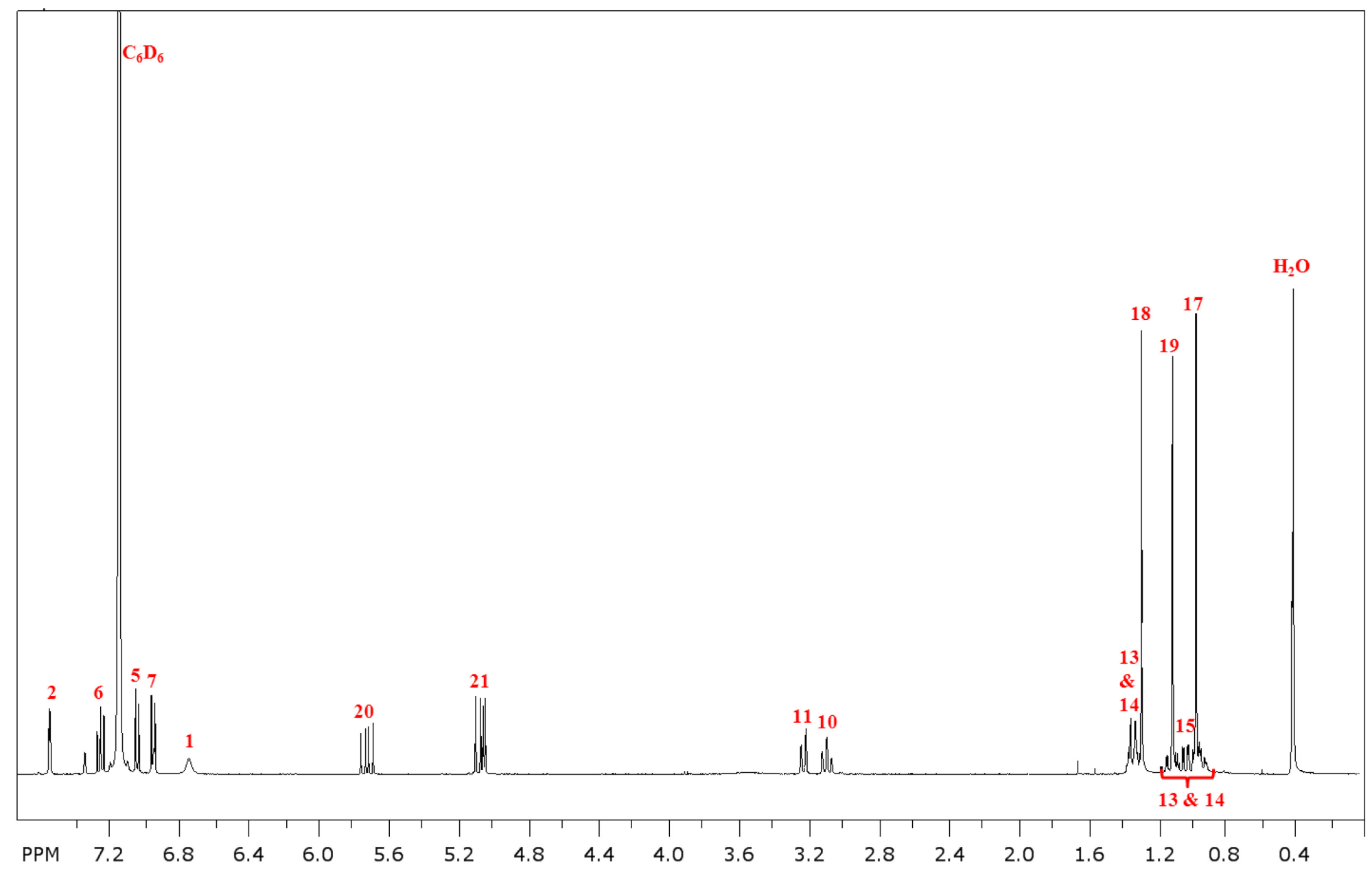

Figure 27: ${ }^{1} \mathrm{H}$ NMR of 1 in $C_{6} D_{6}$ 


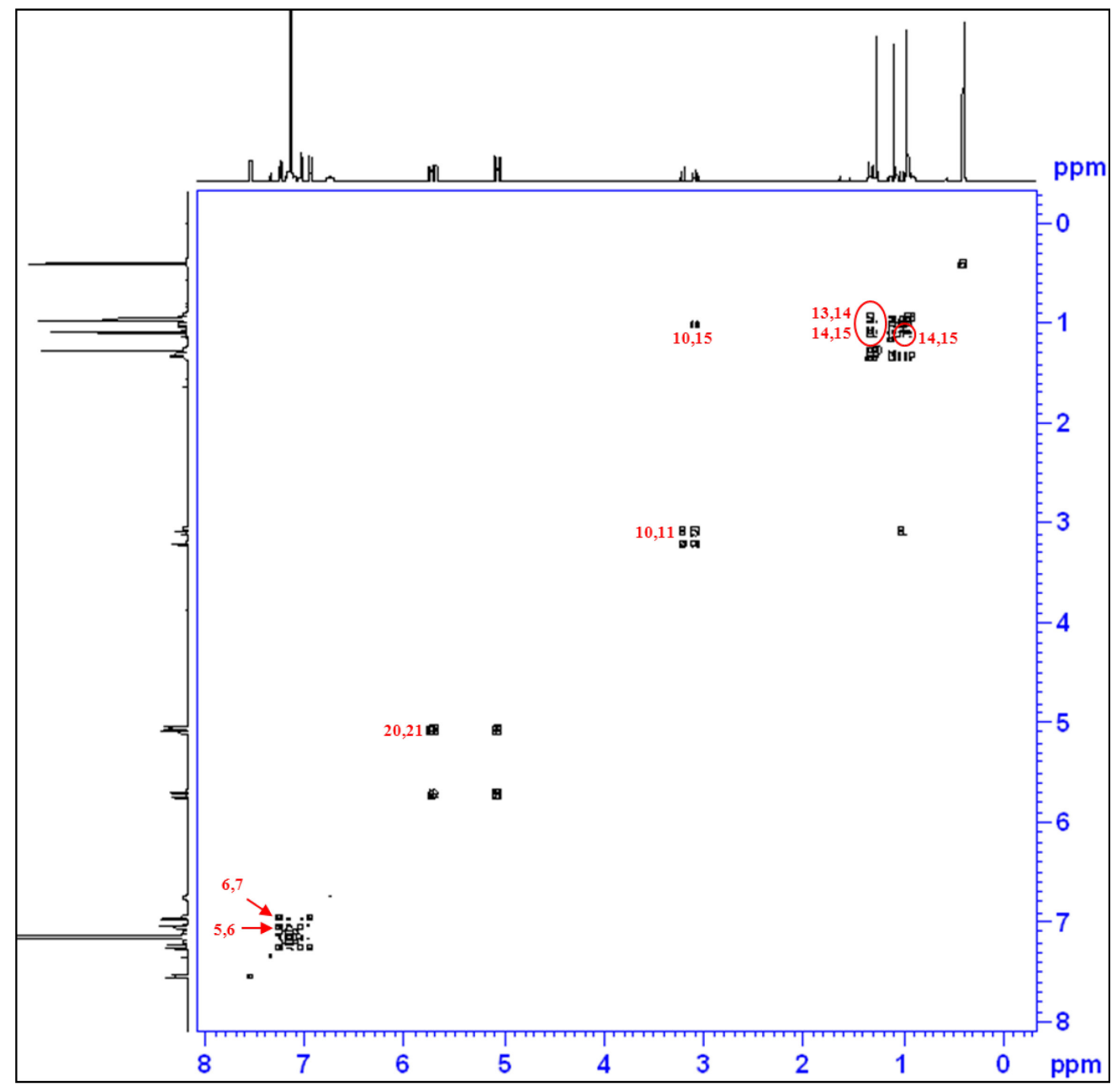

Figure 28:COSY of 1 in $C_{6} D_{6}$ 


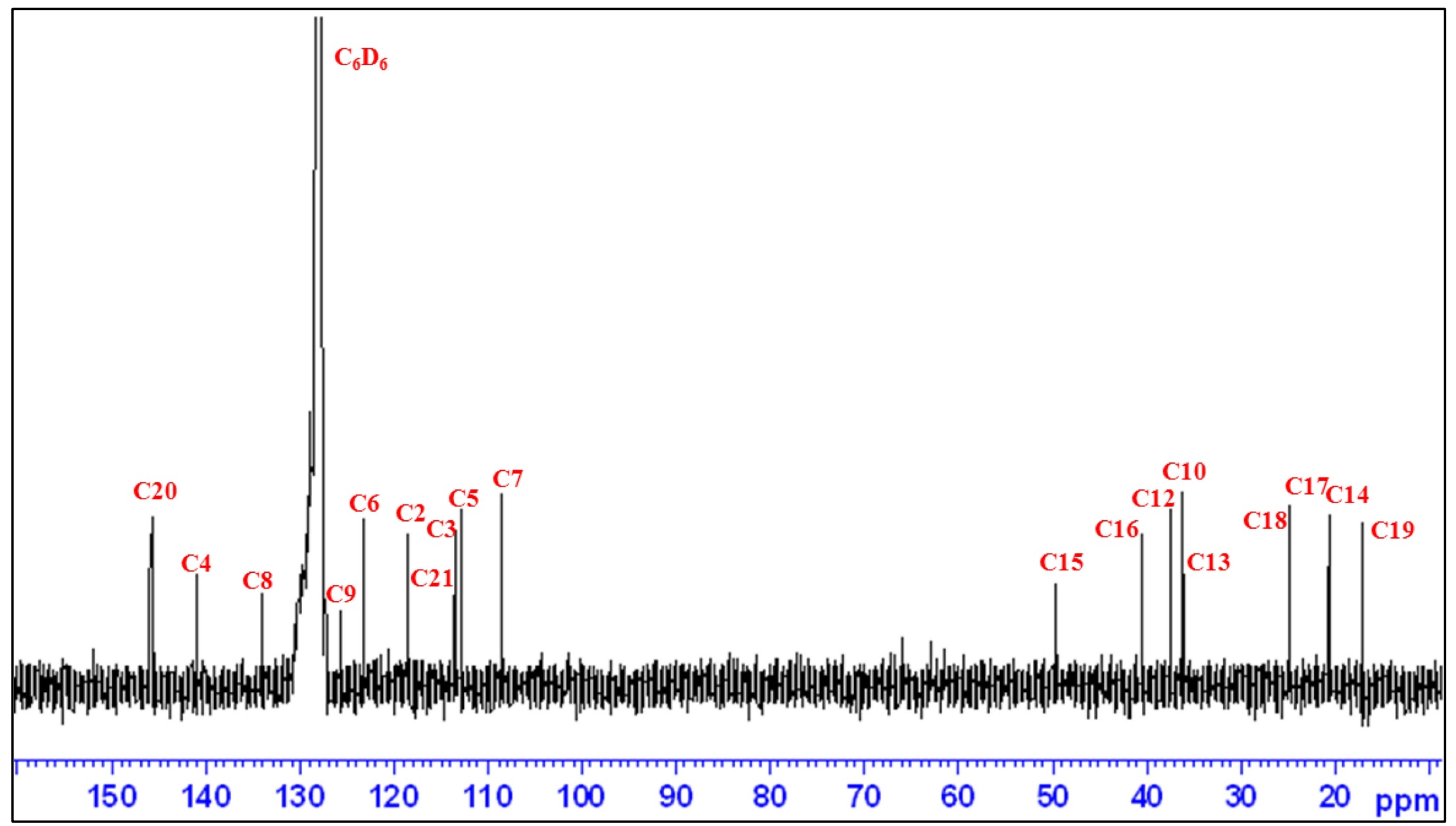

Figure 29:C13 NMR of 1 in $C_{6} D_{6}$ 


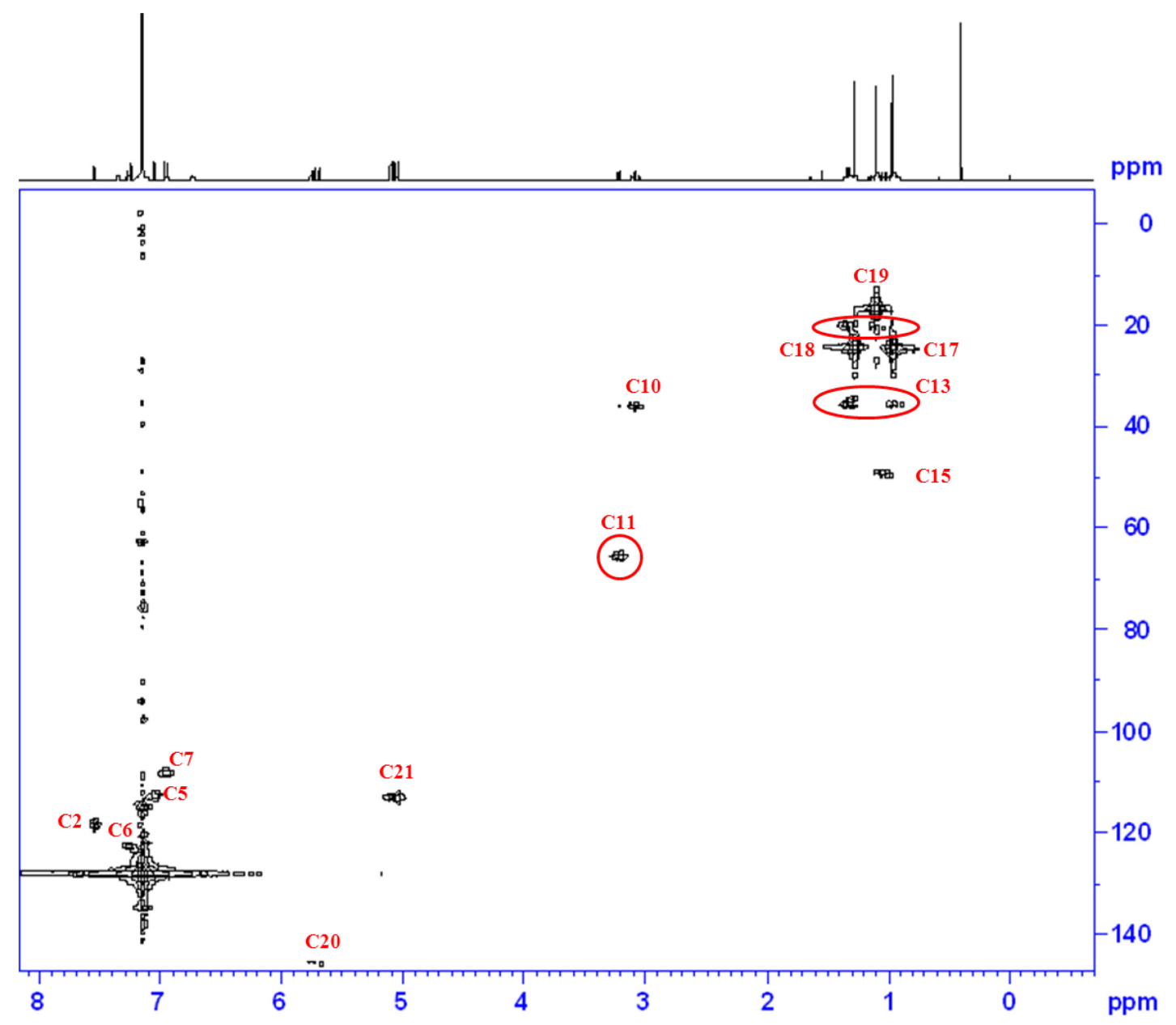

Figure 30: $\mathrm{HMQC}$ of 1 in $\mathrm{C}_{6} \mathrm{D}_{6}$

The assignment of the carbons and hydrogens was done using the numbering utilized by Moore et al., (1987) with hapalindoles that had a mass of 304, an isonitrile group, and bonding of the indole moiety at positions 3 and 4 . Three hapalindoles were found that fulfilled the criteria, these included, Hapalindole $\mathrm{H}$ Isonitrile, 12-epiHapalindole H Isonitrile, and 12-epi-Hapalindole J Isonitrile and are compared in Table 7. The compound Hapalindole $\mathrm{J}$ Isonitrile also fulfilled the criteria, but the ${ }^{13} \mathrm{C} \mathrm{NMR}$ data was not reported by Moore et al., (1987). In order to compare 1 with previously identified hapalindoles the assignments of the carbons was done on the basis of the chemical shift. Compound $\mathbf{1}$ was closely resembled 12-epi-Hapalindole H Isonitrile 
when comparing the 13C NMR data, but further evaluation of the proton data would be necessary to determine the structure. From this comparison 1 was suggested to be 12 epi-Hapalindole H Isonitrile (Figure 31).

Table 7: ${ }^{13}$ C NMR Chemical Shifts of 1, Hapalindole H Isonitrile, 12-epi-Hapalindole H Isonitrile, and 12-epi-Hapalindole J Isonitrile

\begin{tabular}{|c|c|c|c|c|}
\hline $\begin{array}{l}\text { Carbon } \\
\text { Position }\end{array}$ & $\begin{array}{l}\text { Compound } 1 \\
\left(\mathrm{C}_{6} \mathrm{D}_{6}\right)\end{array}$ & $\begin{array}{c}\text { Hapalindole } \mathbf{H} \\
\left(\mathrm{CHCl}_{3}\right) \\
\text { Moore et al., } 1987\end{array}$ & $\begin{array}{c}\text { 12-epi- } \\
\text { Hapalindole } \mathrm{H} \\
\left(\mathrm{CHCl}_{3}\right) \\
\text { Klein et al., } 1995\end{array}$ & $\begin{array}{c}\text { 12-epi-Hapalindole } \mathbf{J} \\
\left(\mathrm{CHCl}_{3}\right) \\
\text { Becher et al., } 2007\end{array}$ \\
\hline 2 & 119.3 & 118.3 & 118.4 & 120.1 \\
\hline 3 & 114.0 & 113.0 & 113.4 & 109.5 \\
\hline 4 & 141.4 & 140.5 & 140.7 & 138.6 \\
\hline 5 & 113.6 & 112.5 & 112.8 & 113.8 \\
\hline 6 & 123.7 & 108.1 & 122.6 & 123.1 \\
\hline 7 & 109.2 & 122.6 & 108.2 & 108.2 \\
\hline 8 & 134.6 & 133.2 & 133.5 & 133.3 \\
\hline 9 & 126.4 & 124.9 & 125.1 & 123.3 \\
\hline 10 & 36.9 & 36.2 & 35.9 & 37.5 \\
\hline 11 & 66.2 & 67.8 & 65.9 & 63.2 \\
\hline 12 & 37.9 & 37.3 & 37.4 & 38.4 \\
\hline 13 & 36.6 & 36.1 & 35.9 & 31.5 \\
\hline 14 & 21.2 & 20.8 & 20.6 & 25.3 \\
\hline 15 & 50.2 & 49.7 & 49.7 & 43.7 \\
\hline 16 & 41.2 & 40.5 & 40.5 & 37.7 \\
\hline 17 & 25.3 & 24.5 & 24.7 & 24.9 \\
\hline 18 & 25.5 & 24.8 & 24.9 & 31.7 \\
\hline 19 & 17.6 & 27.2 & 17.0 & 27.9 \\
\hline 20 & 146.5 & 138.5 & 145.3 & 143.7 \\
\hline 21 & 114.2 & 115.8 & 133.6 & 111.3 \\
\hline 23 & n.d & 157.5 & 157.5 & n.d. \\
\hline
\end{tabular}




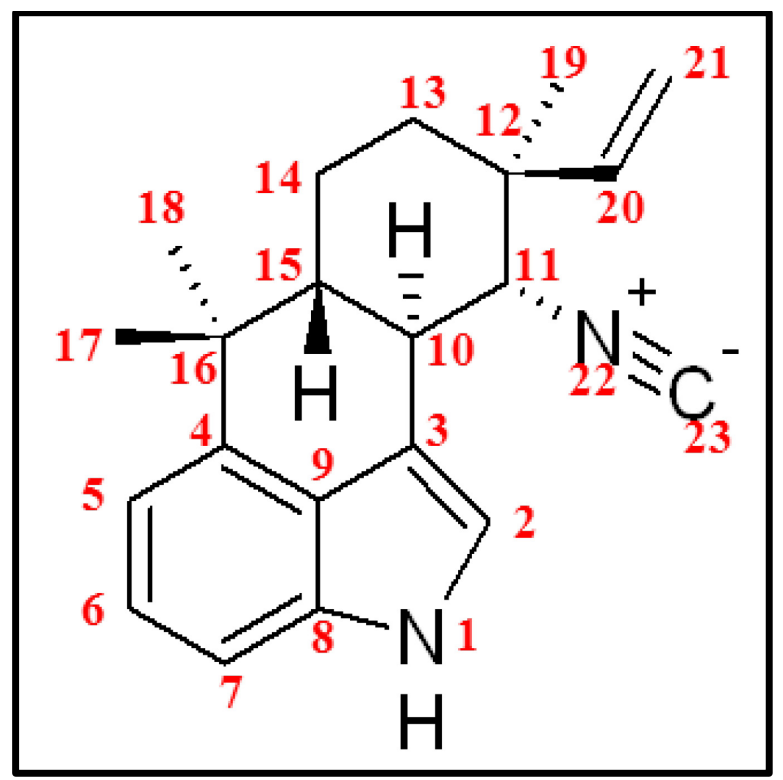

Figure 31: 12-epi-Hapalindole H Isonitrile Structure (Moore et al., 1987)

The assignment of the protons was done by the HMQC spectrum, where the assigned carbons were correlated to hydrogen chemical shifts. The strategy was necessary for some of the protons in the cyclohexane ring which had overlapping chemical shifts. The results of these assignments and comparison to four hapalindoles, Hapalindole H Isonitrile, Hapalindole J Isonitrile, 12-epi-Hapalindole H Isonitrile, and 12-epi-Hapalindole J Isonitrile are presented, in Table 8. 
Table 8: ${ }^{1} \mathrm{H}$ NMR Comparison of 1 and Four Previously Identified Hapalindoles (400 $\mathrm{MHz}, \mathrm{J}$ in $\left.\mathrm{Hz}\right)$

\begin{tabular}{|c|c|c|c|c|c|}
\hline $\begin{array}{l}\text { Proton } \\
\text { Position }\end{array}$ & $\begin{array}{l}\text { Compound } 1 \\
\left(\mathrm{C}_{6} \mathrm{D}_{6}\right)\end{array}$ & $\begin{array}{c}\text { Hapalindole } \mathrm{H} \\
\left(\mathrm{CHCl}_{3}\right) \\
\text { Moore et al., } 1987\end{array}$ & $\begin{array}{c}\text { Hapalindole } \mathbf{J} \\
\quad\left(\mathrm{CHCl}_{3}\right) \\
\text { Moore et al., } 1987\end{array}$ & $\begin{array}{c}\text { 12-epi-Hapalindole } \mathrm{H} \\
\left(\mathrm{CHCl}_{3}\right) \\
\text { Klein et al., } 1995\end{array}$ & $\begin{array}{c}\text { 12-epi- } \\
\text { Hapalindole J } \\
\left(\mathrm{CHCl}_{3}\right) \\
\text { Becher et al., } \\
2007\end{array}$ \\
\hline 1 & 6.75 br s & $8.04 \mathrm{br}$ & $8.00 \mathrm{br}$ & 8.02 brs & $7.98 \mathrm{br}$ \\
\hline 2 & $7.55 \mathrm{dd}(1.8,2)$ & $7.62 \mathrm{dd}$ & $6.88 \mathrm{brt}$ & $7.59 \mathrm{dd}$ & $6.77 \mathrm{br}$ \\
\hline 5 & $7.05 \mathrm{~d}(7.3)$ & $7.02 \mathrm{~m}$ & $6.92 \mathrm{~m}$ & $7.03 \mathrm{~m}$ & $6.95 \mathrm{~d}$ \\
\hline 6 & $7.26 \mathrm{dd}(7.3,8)$ & $7.18 \mathrm{~m}$ & $7.17 \mathrm{~m}$ & $7.18 \mathrm{~m}$ & $7.158 \mathrm{dd}$ \\
\hline 7 & $6.96 \mathrm{~d}(8)$ & $7.19 \mathrm{~m}$ & $7.18 \mathrm{~m}$ & $7.18 \mathrm{~m}$ & $7.159 \mathrm{~d}$ \\
\hline $10 \mathrm{ax}$ & $3.09 \mathrm{dd}(10.7,10.7)$ & $3.17 \mathrm{td}$ & & $3.23 \mathrm{dd}$ & \\
\hline $10 \mathrm{eq}$ & & & 3.85 br m & & 3.83 br d \\
\hline $11 \mathrm{ax}$ & 3.21 br d (10.7) & $3.51 \mathrm{brdtd}$ & & $3.54 \mathrm{~d}$ & \\
\hline $11 \mathrm{eq}$ & & & 4.17 brd & & $4.21 \mathrm{br}$ \\
\hline $13 \mathrm{ax}$ & $0.97 \mathrm{~m}$ & $1.38 \mathrm{dt}$ & $1.88 \mathrm{td}$ & $1.45 \mathrm{ddd}$ & $1.68 \mathrm{td}$ \\
\hline $13 \mathrm{eq}$ & $1.33 \mathrm{~m}$ & $2.07 \mathrm{dt}$ & $1.37 \mathrm{br} \mathrm{dt}$ & 1.78 ddd & $1.56 \mathrm{br} \mathrm{dt}$ \\
\hline $14 \mathrm{ax}$ & $1.15 \mathrm{~m}$ & $1.66 \mathrm{qd}$ & $1.11 \mathrm{brq}$ & 1.59 dddd & 1.07 n.d. \\
\hline $14 \mathrm{eq}$ & $1.33 \mathrm{~m}$ & $1.79 \mathrm{dq}$ & $1.72 \mathrm{br} \mathrm{dq}$ & 1.85 dddd & $1.50 \mathrm{~m}$ \\
\hline $15 \mathrm{ax}$ & $1.03 \mathrm{~m}(2.8,10.7)$ & 1.52 ddd & $2.10 \mathrm{dt}$ & 1.49 ddd & $2.11 \mathrm{dt}$ \\
\hline 17 & $0.96 \mathrm{~s}$ & $1.45 \mathrm{~s}$ & $1.51 \mathrm{~s}$ & $1.15 \mathrm{~s}$ & $1.50 \mathrm{~s}$ \\
\hline 18 & $1.28 \mathrm{~s}$ & $1.11 \mathrm{~s}$ & $1.21 \mathrm{~s}$ & $1.46 \mathrm{~s}$ & $1.22 \mathrm{~s}$ \\
\hline 19 & $1.10 \mathrm{~s}$ & $1.28 \mathrm{~s}$ & $0.80 \mathrm{~s}$ & $1.32 \mathrm{~s}$ & $1.23 \mathrm{~s}$ \\
\hline 20 & $5.73 \mathrm{dd}(10.8,17.4)$ & 6.29 ddd & $6.04 \mathrm{dd}$ & $5.89 \mathrm{dd}$ & $5.57 \mathrm{dd}$ \\
\hline $21 \mathrm{E}$ & $5.08 \mathrm{dd}(0.8,17.4)$ & 5.36 ddd & $5.13 \mathrm{brd}$ & $5.20 \mathrm{~d}$ & $4.61 \mathrm{~d}$ \\
\hline $21 \mathrm{Z}$ & $5.06 \mathrm{dd}(0.8,10.8)$ & $5.29 \mathrm{dd}$ & $5.07 \mathrm{brd}$ & $5.17 \mathrm{~d}$ & $4.75 \mathrm{~d}$ \\
\hline
\end{tabular}

In the ${ }^{1} \mathrm{H}$ NMR obtained in $\mathrm{MeOD}$ and $\mathrm{D}_{2} \mathrm{O}$, the aromatic protons at $7.39,7.06$, 6.98 , and $6.85 \delta$ were assigned to positions $2,5,6$, and 7 . The singlet that corresponds to the amine proton in the indole moiety produced a lower intensity presumptively as a result of deuterium exchange with the $\mathrm{D}_{2} \mathrm{O}$ solvent that was added to the sample to facilitate dissolution of $\mathbf{1}$ in MeOD. Figure 32 illustrates the coupling between the protons at the 5,6 , and 7 positions on the benzoid ring of the indole moiety. 


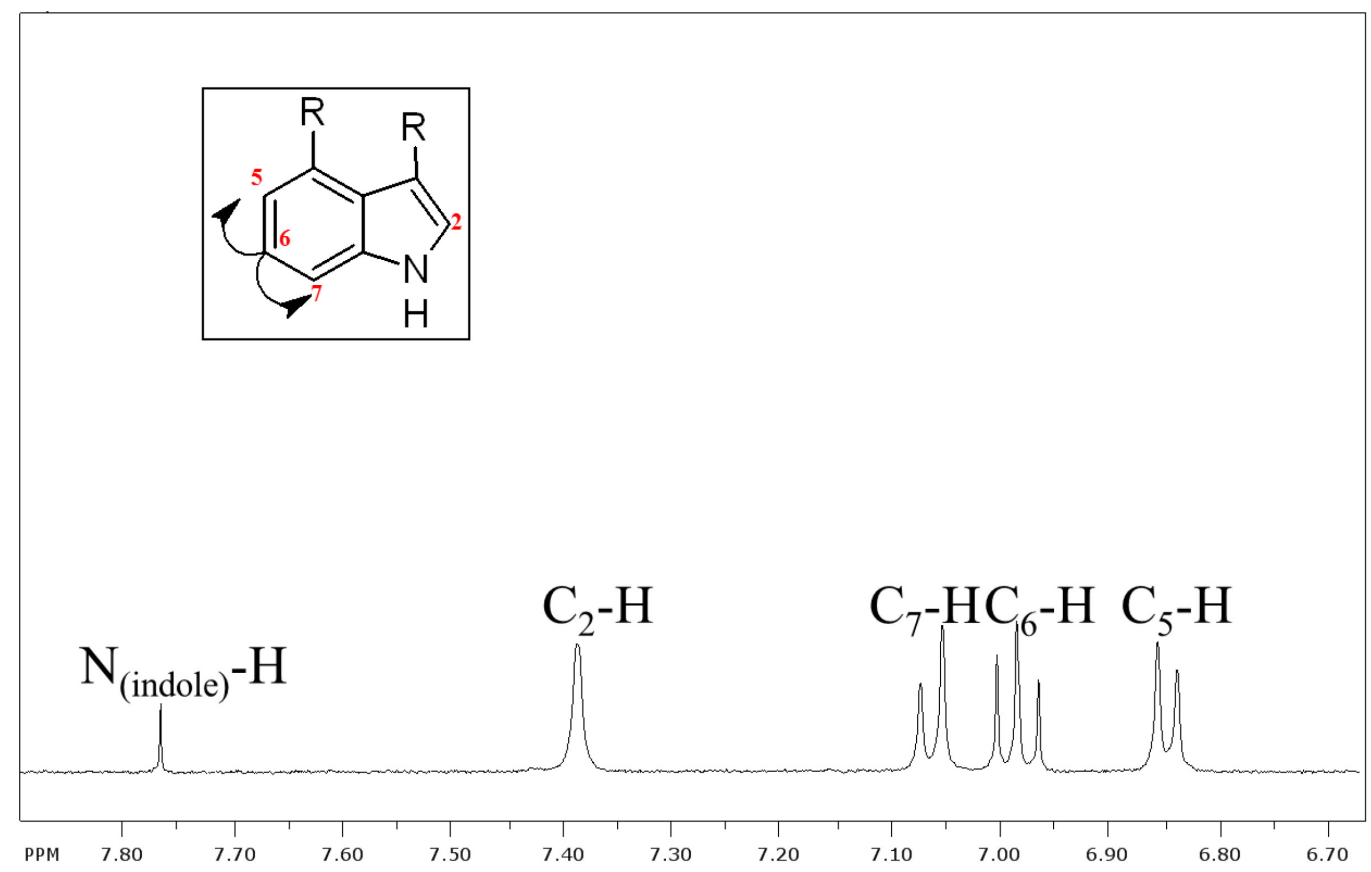

Figure 32: Aromatic Region of ${ }^{1} \mathrm{H} N M R$ of 1 in $\mathrm{MeOH}$ and $\mathrm{D}_{2} \mathrm{O}$

Similar splitting patterns observed in the aromatic region of the MeOD proton NMR are observed in the ${ }^{1} \mathrm{H}$ NMR conducted in $\mathrm{C}_{6} \mathrm{D}_{6}$ (Figure 32). The proton at $\mathrm{C} 2$ was split in the $\mathrm{C}_{6} \mathrm{D}_{6}$ spectrum into a doublet of doublet as was observed by Moore et al., (1987) for Hapalindole H Isonitrile, and Klein et al., (1995) for 12-epi-Hapalindole H Isonitrile. Moore et al., (1987) deduced that the vicinal coupling to the N1 proton on the indole, and long range coupling to the proton at $\mathrm{C} 10$, caused a doublet of doublets. The coupling constant calculated for splitting between the protons at C6 and C5 was $7.3 \mathrm{~Hz}$. The coupling constant calculated for protons on $\mathrm{C} 6$ and $\mathrm{C} 7$ was $8 \mathrm{~Hz}$. The coupling constants for these protons was not calculated for 12-epi-Hapalindole H Isonitrile by Klein et al., (1995) most likely because of the fact that Klein observed multiplets instead of a distinguishable splitting pattern. Moore et al., (1987), however, calculated values of $7.2 \mathrm{~Hz}$ for the coupling between the protons at $\mathrm{C} 5$ and $\mathrm{C} 6$, and $8.2 \mathrm{~Hz}$ between the protons at $\mathrm{C} 6$ and $\mathrm{C} 7$ in Hapalindole $\mathrm{H}$ Isonitrile. Thus the coupling constants for 
Hapalindole H Isonitrile were comparable to the values calculated for $\mathbf{1}$. It was shown in the COSY (Figure 28) that the doublet of doublet produced by the proton on C6 coordinates to the both doublets produced by the protons on C5 and C7. The doublets of C5 and C7 were shown to only slightly inter-coordinate. The doublet of doublets produced by the proton at $\mathrm{C} 2$ was not shown to coordinate to any other protons, this could corroborate the $\mathrm{C} 2$ assignment since the corresponding hydrogens of $\mathrm{C} 2$ would not show correlation in the COSY. One main discrepancy between the ${ }^{1} \mathrm{H}$ NMR data for $\mathbf{1}$ and the reported 12-epi Hapalindole J Isonitrile and 12-epi- Hapalindole H Isonitrile NMR data, is the chemical shift of the singlet produced by the proton attached to the indole nitrogen. The change of the chemical environment, as a result of the difference of solvent most likely caused the change in chemical shift.

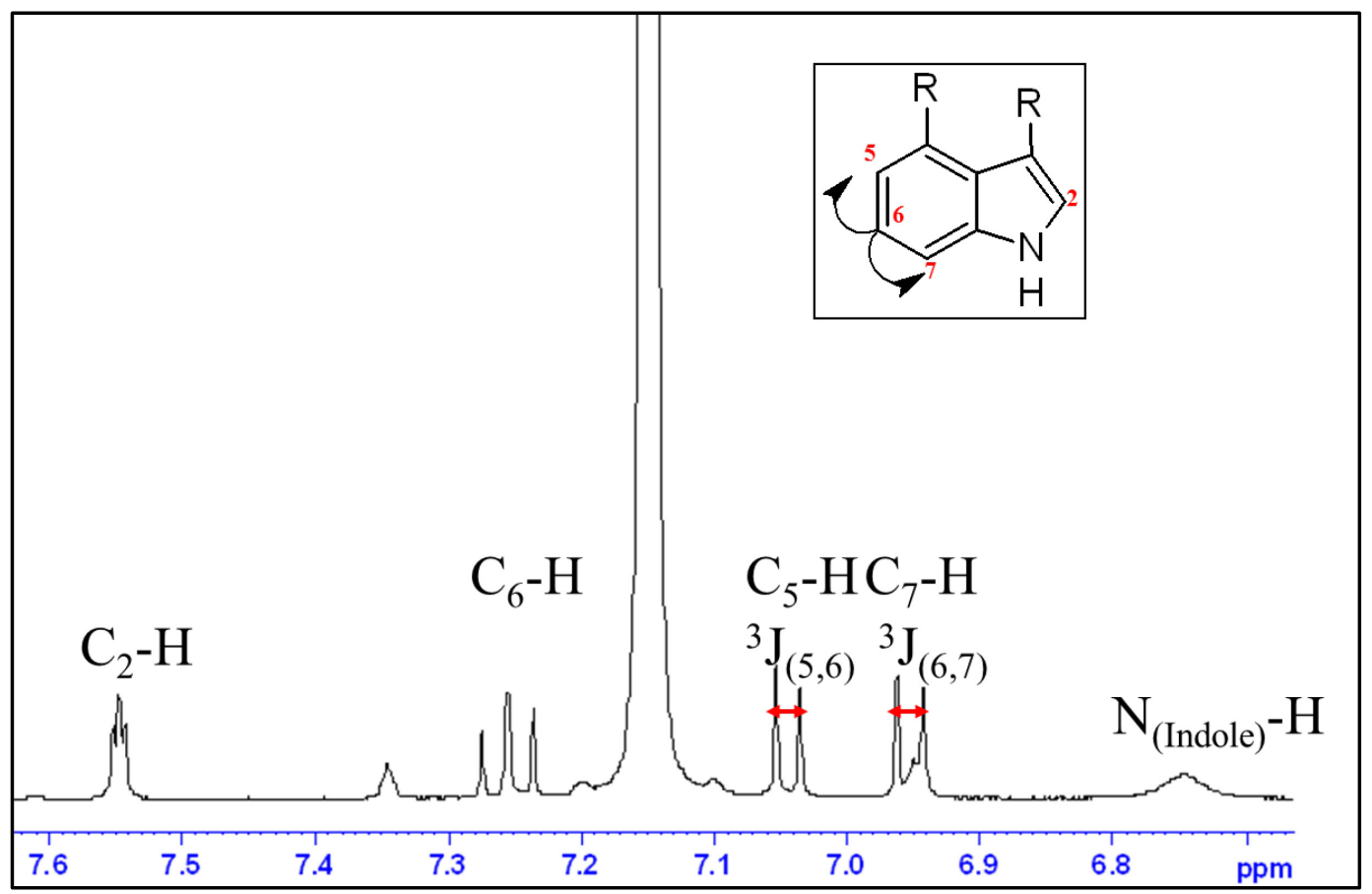

Figure 33: Proton NMR of 1 in $C_{6} D_{6}$ 
The terminal alkene substituent at the $\mathrm{C} 20,21$ position in the hapalindole structure was confirmed by the three sets of doublet of doublets each inter-correlating in the $2 \mathrm{D}$ COSY experiment (Figure 34). The doublet of doublets at $5.73 \delta$ was integrated to 1 proton and was shown to correlate to the two doublets of doublets at 5.06 and $5.08 \delta$ in the COSY spectrum each of which also integrated to one proton. The coupling constants calculated for the doublet of doublet at $5.73 \delta$ were $17.4 \mathrm{HZ}$ and $10.8 \mathrm{~Hz}$. The coupling constant at $17.4 \mathrm{~Hz}$ suggests a coupling between the proton on $\mathrm{C} 20$ and the proton at the E position on $\mathrm{C} 21$. A value of $17.4 \mathrm{~Hz}$ was also calculated in the splitting of the doublet of doublet at $5.08 \delta$ suggesting that this doublet of doublet was produced by the $\mathrm{C} 21 \mathrm{E}$ proton. The doublet of doublet at $5.06 \delta$ produced a coupling constant of $10.8 \mathrm{~Hz}$ which suggests that the doublet of doublet was produced by the $\mathrm{C} 21 \mathrm{Z}$ proton. Coupling between the protons at $\mathrm{C} 21$ was observed and caused splitting in the doublet of doublets at 5.06 and $5.08 \delta$ with a coupling constant of $0.8 \mathrm{~Hz}$ (Figure 35). 


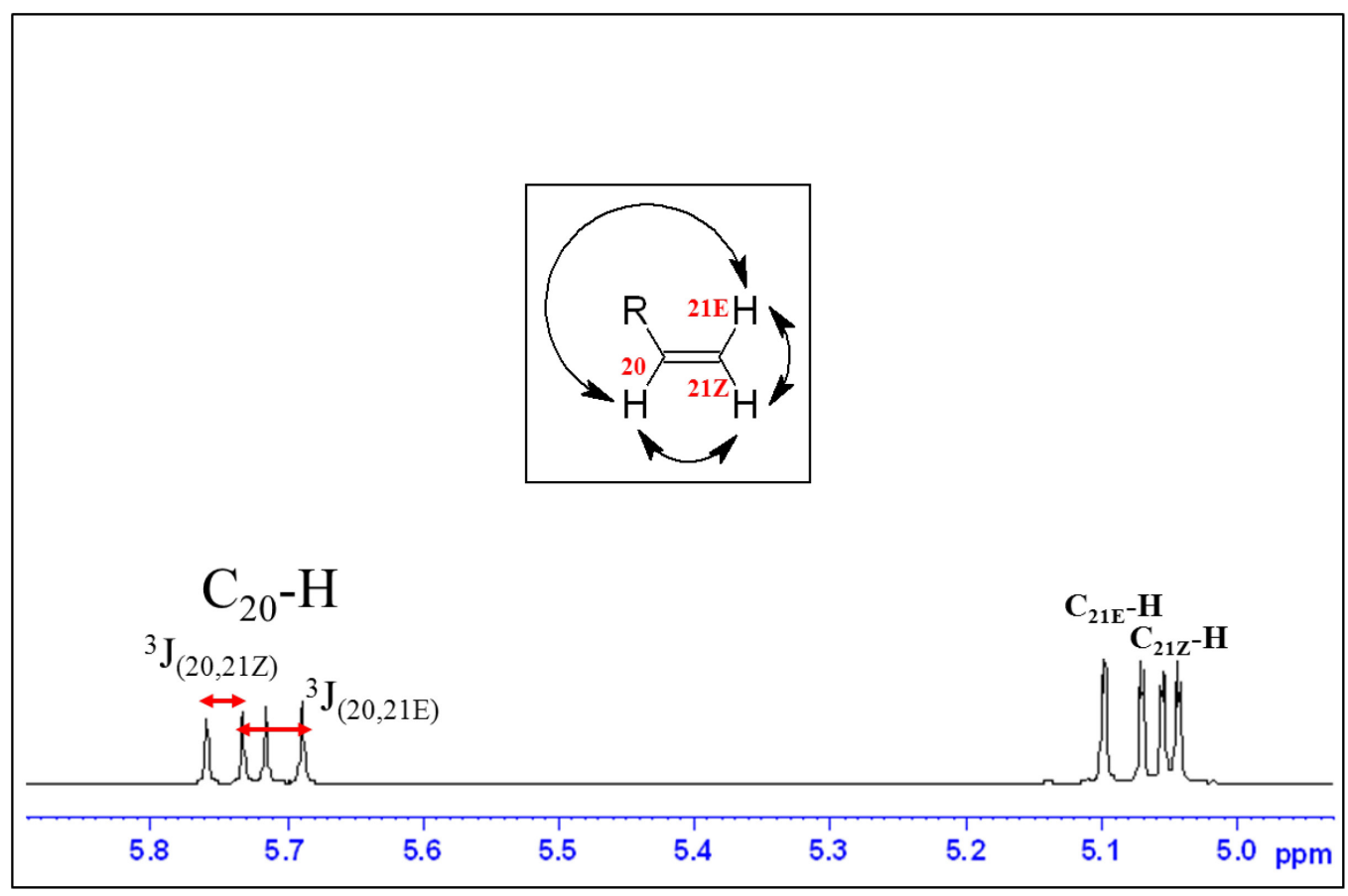

Figure 34: Unsaturated Region of ${ }^{1} \mathrm{H} N M R$

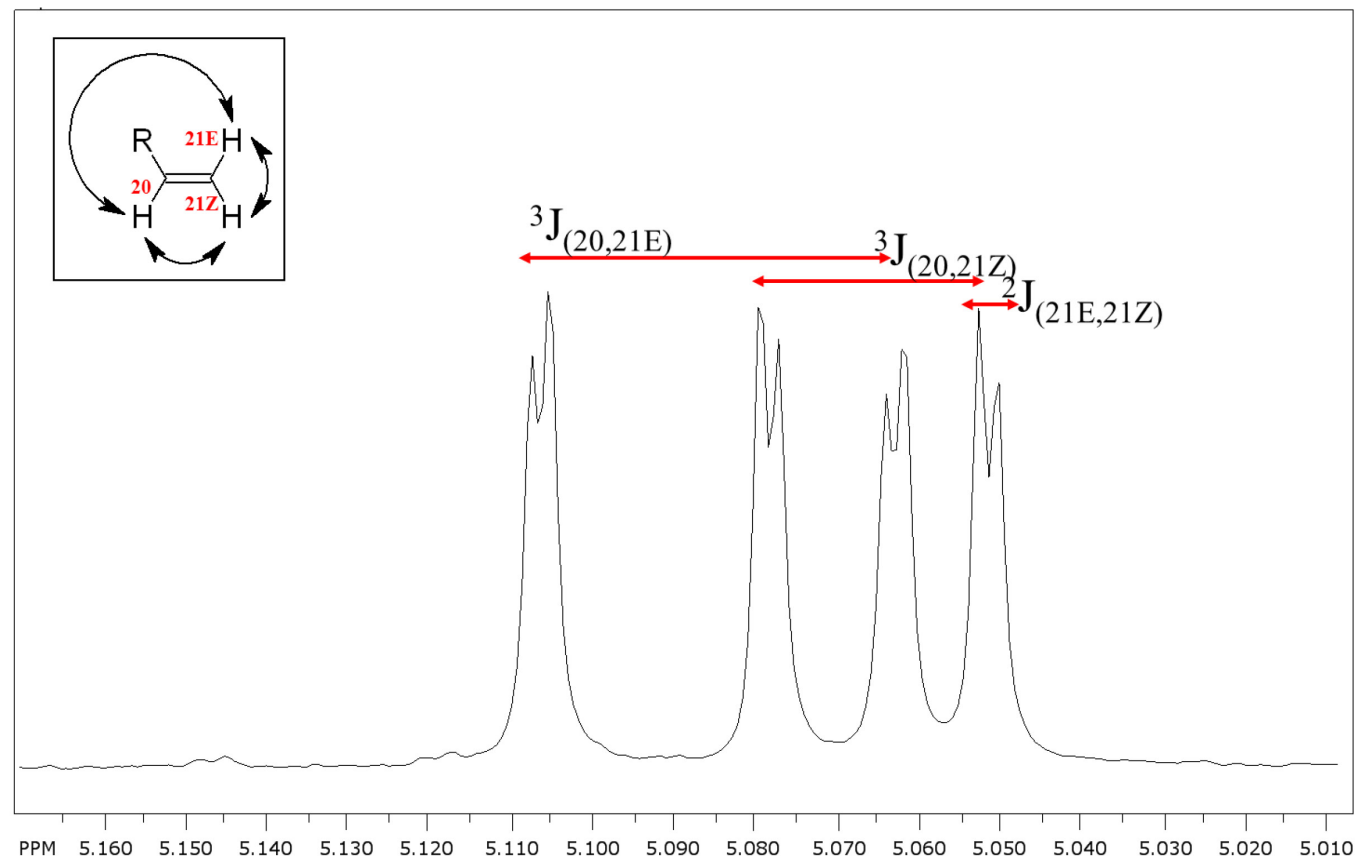

Figure 35: Chemical Shifts of C21 Protons in 1 
The coupling constants and COSY were key to determine the protons on C10, 11, 13,14 , and 15 . The broad doublet at $3.21 \delta$ was shown to correlate to the broad doublet of doublet at $3.09 \delta$. The chemical shift of the broad doublet corresponds to a proton attached to the same carbon as an isonitrile group which is commonly observed in the hapalindole family. The calculated coupling of $10.7 \mathrm{~Hz}$ for the doublet of doublet and the doublet corresponded to diaxial interactions, further confirming the base hapalindole skeleton with an isonitrile attached to a cyclohexane ring (Pavia et al., 2009). The doublet was assigned as the proton on $\mathrm{C} 11$ and the doublet of doublets was assigned as the proton on $\mathrm{C} 10$ on the basis of HMQC data. The HMQC correlated the hydrogens to the carbons assigned as $\mathrm{C} 10$ and $\mathrm{C} 11$ on the basis of the relative chemical shifts. Given the coupling constants that correspond to diaxial coupling, the protons on $\mathrm{C} 11, \mathrm{C} 10$, and C15 were determined to all be axial (Figure 36). This conformation would lead to the relative configuration of $10 \mathrm{~S}^{*}, 11 \mathrm{~S}^{*}$, and $15 \mathrm{~S}^{*}$.

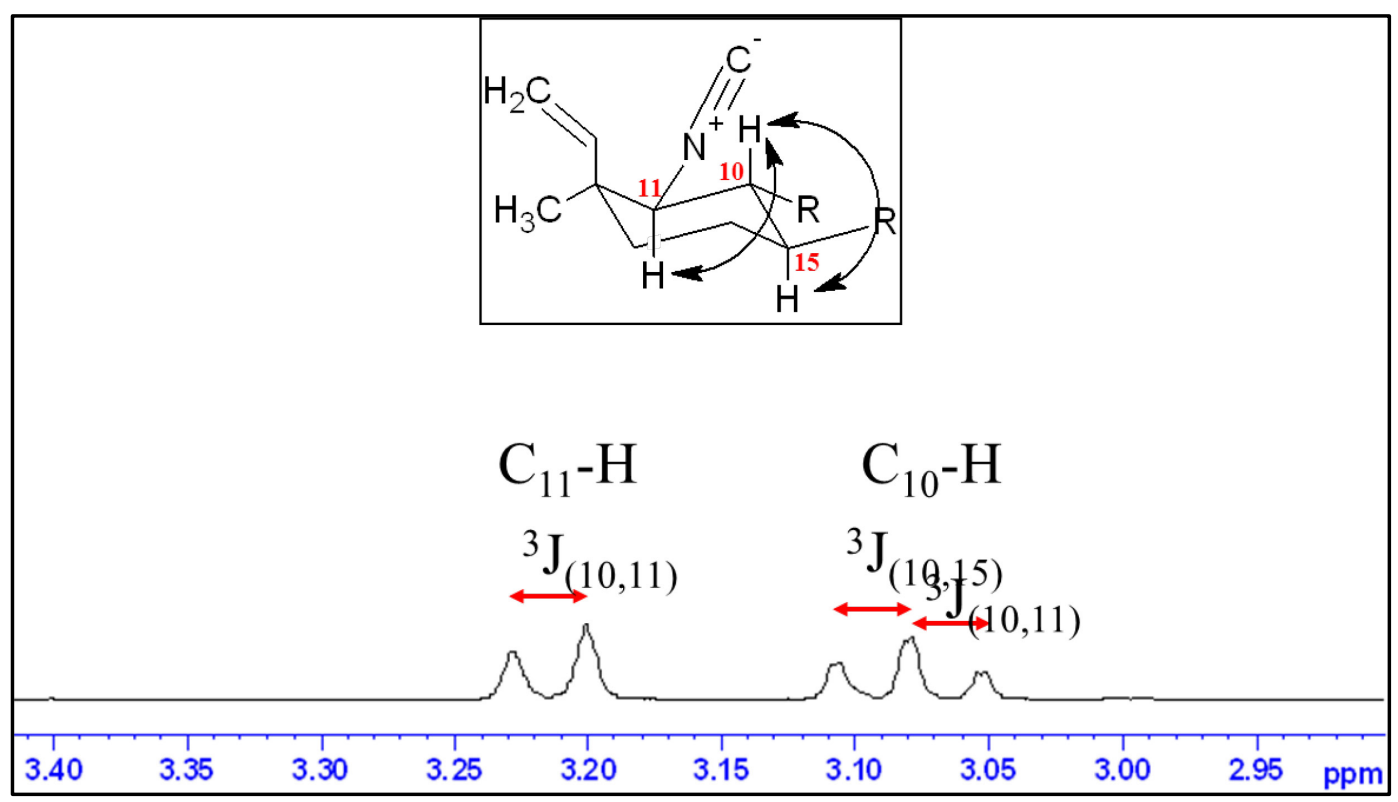

Figure 36: ${ }^{1} \mathrm{H}$ NMR of C10 and C11 Protons in 1 
The aliphatic region of the ${ }^{1} \mathrm{H}$ NMR in $\mathrm{C}_{6} \mathrm{D}_{6}$ was difficult to analyze because of the large degree of overlapping of the chemical shifts (Figure 38). From the COSY it was determined that the doublet of doublets at $3.09 \delta$ coupled to a multiplet at $1.03 \delta$. The doublet of doublets was deduced to be the hydrogen on the $\mathrm{C} 10$ position of the cyclohexane ring in the hapalindole structure. Using the COSY data it was proposed that the multiplet at $1.03 \delta$ was produced by the hydrogen on C15 as shown in Figure 38 .

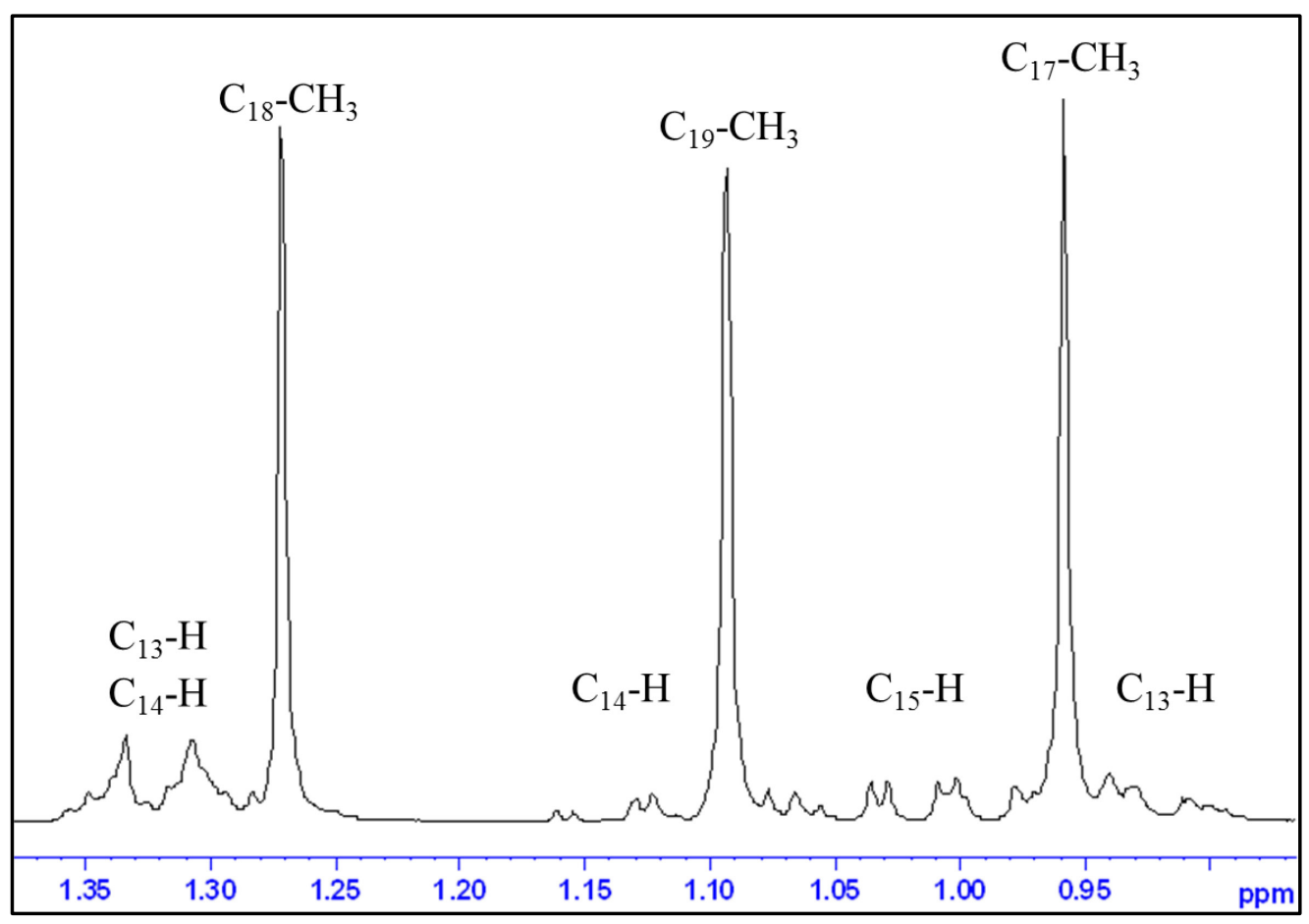

Figure 37: Aliphatic Region of ${ }^{1} H$ NMR of 1 


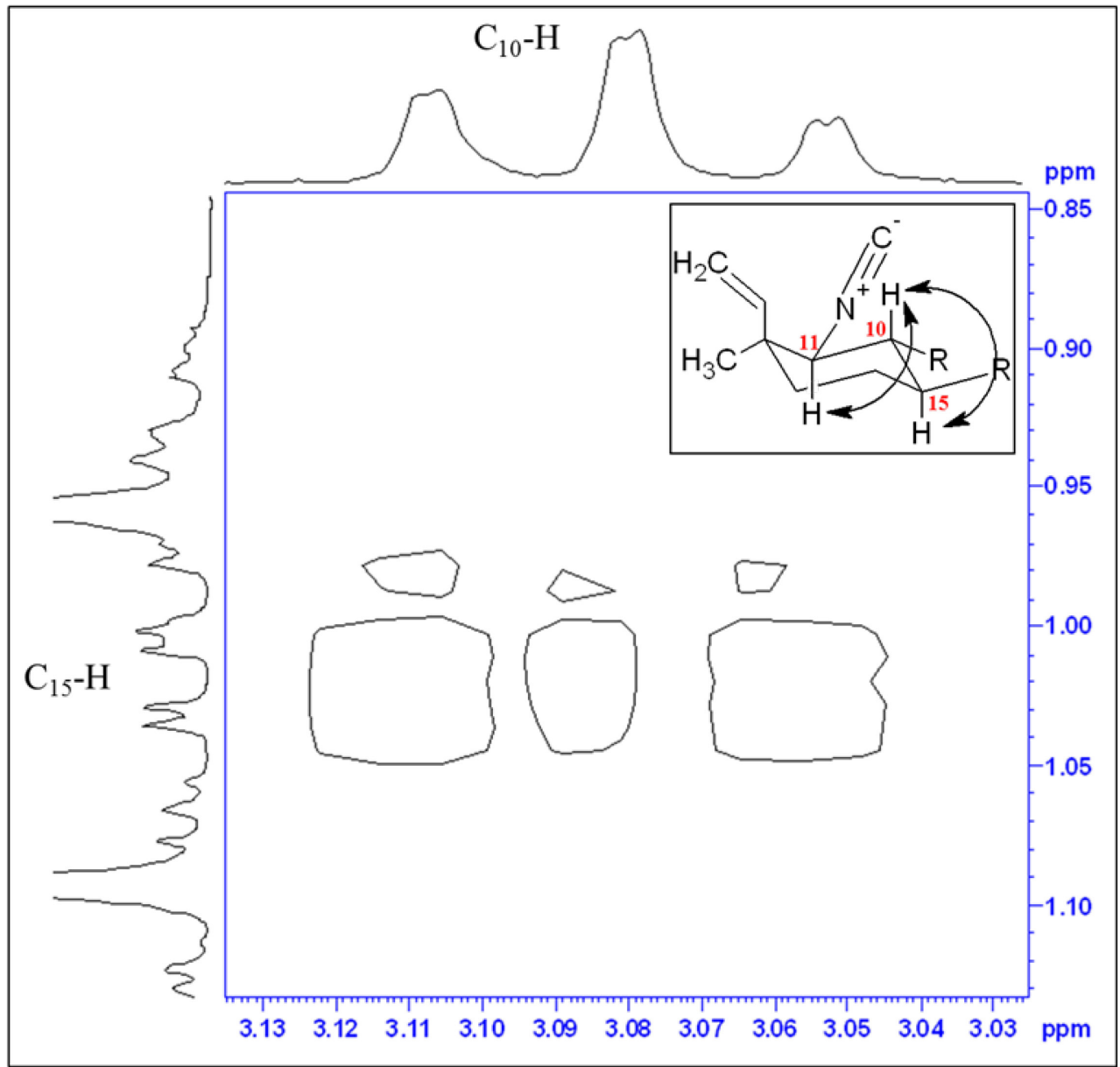

Figure 38: COSY Correlation of Protons of C10 and C15 in 1

In order to determine which of the overlapping multiplets were produced by particular hydrogens the HMQC data was used to correlate proton and carbon signals. The carbon signals were compared to previously identified hapalindoles (Moore et al., 1987; Klein et al., 1995; Becher et al., 2007) and assigned on the basis of the carbon chemical shifts and comparison of proton chemical shifts and splitting. The carbon signal at $36.6 \delta$ was labeled as $\mathrm{C} 13$ as previously assigned for other hapalindole structures and the signal at $21.2 \delta$ was assigned as $\mathrm{C} 14$. The doublet of multiplets at $1.33 \delta$ was shown to correlate in the HMQC to both $\mathrm{C} 13$ and $\mathrm{C} 14$ (Figure 39). The overlapping doublet of multiplets might be a result of the doublets of triplets and the doublets of quartets 
produced by the equatorial hydrogens on C13 and C14 observed by Klein et al., (1995). The chemical shifts of these multiplets were higher than the other multiplets that were produced by the remaining hydrogens on $\mathrm{C} 13$ and $\mathrm{C} 14$. The splitting and the chemical shifts suggest that these protons are the equatorial $\mathrm{C} 13$ and $\mathrm{C} 14$ positions since the observations by Klein et al., (1995) showed the chemical shift of the equatorial hydrogens to be higher than that of the axial hydrogens. The multiplet at $1.15 \delta$ was assigned as the axial hydrogen on $\mathrm{C} 14$ because of the correlation with the carbon signal at 21.28. The remaining multiplet at $0.97 \delta$ was assigned as the axial $\mathrm{C} 13$ hydrogen because of the correlation with the carbon at $36.6 \delta$.

The singlets at $1.28,1.10,0.96 \delta$ each integrated to 3 hydrogens and were determined to correspond to the three methyl groups observed in the hapalindole family. The chemical shifts produced in the ${ }^{1} \mathrm{H}$ NMR differed greatly from the compared hapalindoles, most likely a result of the difference in solvents. For this reason, the assignment of the three methyl groups was done by correlating the singlets to the carbon signals of C17-19 with the HMQC data. The methyl groups at C17 and C18 were assigned on the basis of the carbon chemical shift shown to correlate to the singlets with the HMQC. The chemical shifts of these two carbons were compared to the previously identified hapalindoles in Table 7. The conformation at the C12 carbon was determined on the basis of the chemical shift of the $\mathrm{C} 19$ carbon. With the chemical shift of $17.6 \delta$, the carbon directly matches the value of 12-epi-Hapalindole H Isonitrile. This suggests that 1 was also a $\mathrm{C} 12$ epimer, and that the relative configuration of C12 was $12 \mathrm{R}^{*}$. 


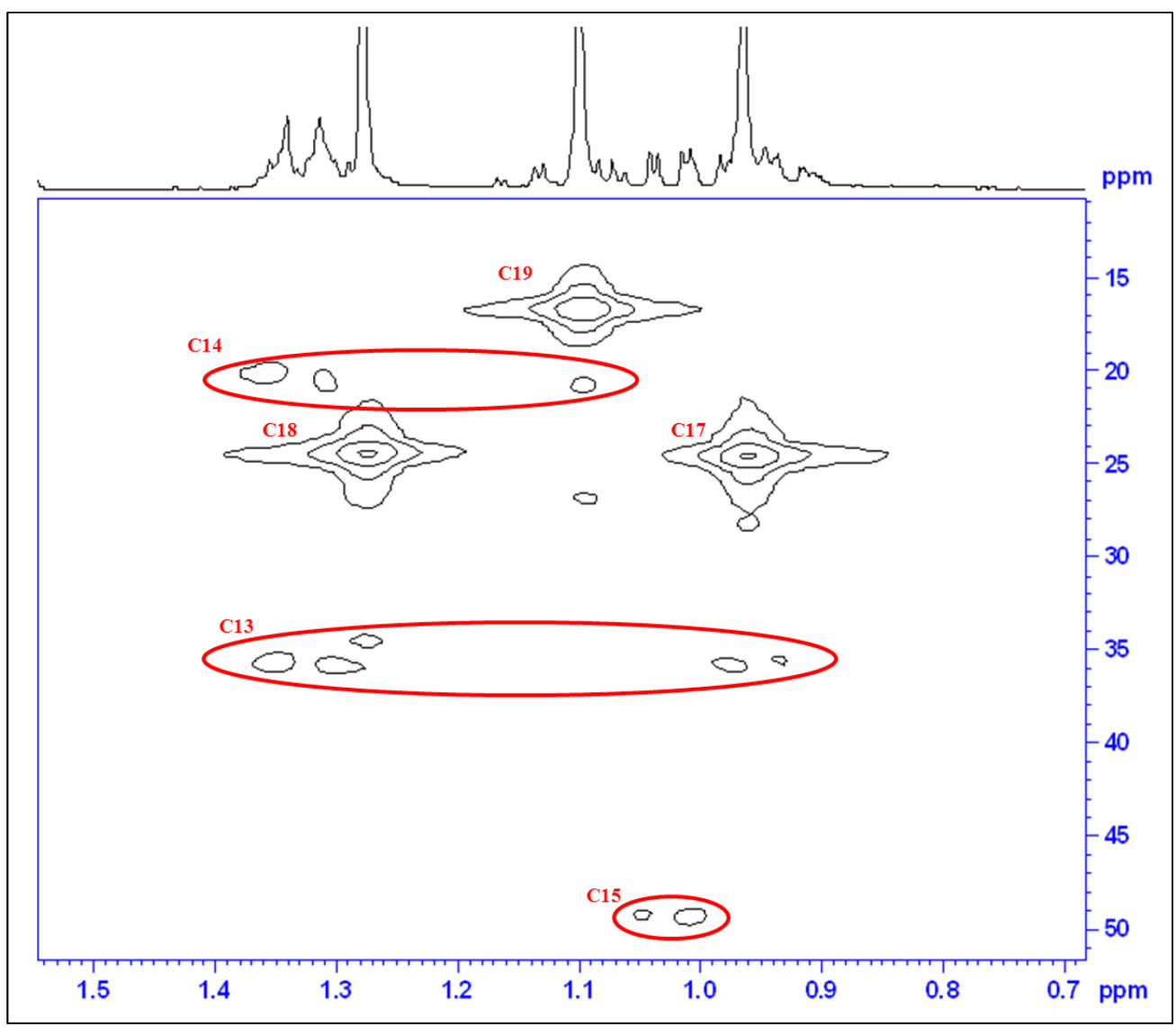

Figure 39: Assignment of Hydrogens using the HMQC of the Aliphatic Region of 1

From the various analyses and experiments using the IR, NMR, and MS, it was determined that 1 was 12-epi-Hapalindole $\mathrm{H}$ Isonitrile. The IR analysis suggested the occurrence of an isonitrile group, the ${ }^{13} \mathrm{C}$ NMR data matched extremely closely to 12 -epiHapalindole $\mathrm{H}$ Isonitrile, and the stereochemistry determined by the ${ }^{1} \mathrm{H}$ NMR matched that observed by Klein et al., (1995). 


\subsubsection{2-epi-Ambiguine B Nitrile}

Compound 2 was isolated as an amorphous white solid from Peak 7. As with 12epi-Hapalindole $\mathrm{H}$ Isonitrile, the mass of Compound $\mathbf{2}$ was deduced by first analyzing the compound using the TSQ Access and then selecting the corresponding ion from the OT Velos data (Figure 40). Compound 2 produced the ion $[\mathrm{M}-\mathrm{H}]^{-}$with a mass of $421 \mathrm{amu}$ in negative mode, and the ion $[\mathrm{M}+\mathrm{H}]^{+}$with a mass of $423 \mathrm{amu}$ in positive mode. The exact mass was able to be selected because of the unique isotope pattern of 3:1, which correlates the presence of a chlorine atom, with the $[\mathrm{M}+\mathrm{H}]^{+}$of $423 \mathrm{~m} / \mathrm{z}$ and $[(\mathrm{M}+2)+\mathrm{H}]^{+}$ of $425 \mathrm{~m} / \mathrm{z}$. The isotope cluster was observed at 17.59 minutes as displayed in Figure 42, which also included the molecular ion at $422 \mathrm{~m} / \mathrm{z}$. The exact $\mathrm{m} / \mathrm{z}$ of the molecular ion was determined to be 422.2128 . The mass was larger than majority of the previously isolated hapalindoles, and suggested that 2 was possibly an ambiguine. The ambiguines, as discussed in Chapter 1, are a group of indole alkaloids that have also been isolated from Stigonemataceae, and were first isolated from Fischerella (Smitka et al., 1992). The fragmentation of the $[\mathrm{M}+\mathrm{H}]^{+}$ion from the OT Velos data produced a number of ions which were proposed to include; $\left[\mathrm{M}-\mathrm{CH}_{3}\right]^{+},[\mathrm{M}-\mathrm{Cl}]^{+},\left[\mathrm{M}-\mathrm{H}_{2} \mathrm{O}\right]^{+}$, and $\left[\mathrm{M}-\left(2 \mathrm{CH}_{3}+\mathrm{CN}\right)\right]^{+}$ (Figure 41). From this data 2 was proposed to be Ambiguine B Isonitrile, given that the formula produced by analyzing the mass spectrometer was $\mathrm{C}_{26} \mathrm{H}_{31} \mathrm{OClN}_{2}$, the same for Ambiguine B Isonitrile. 
F:Mass Specl...120101118_FIU_B_01

11/18/2010 10:35:54 AM

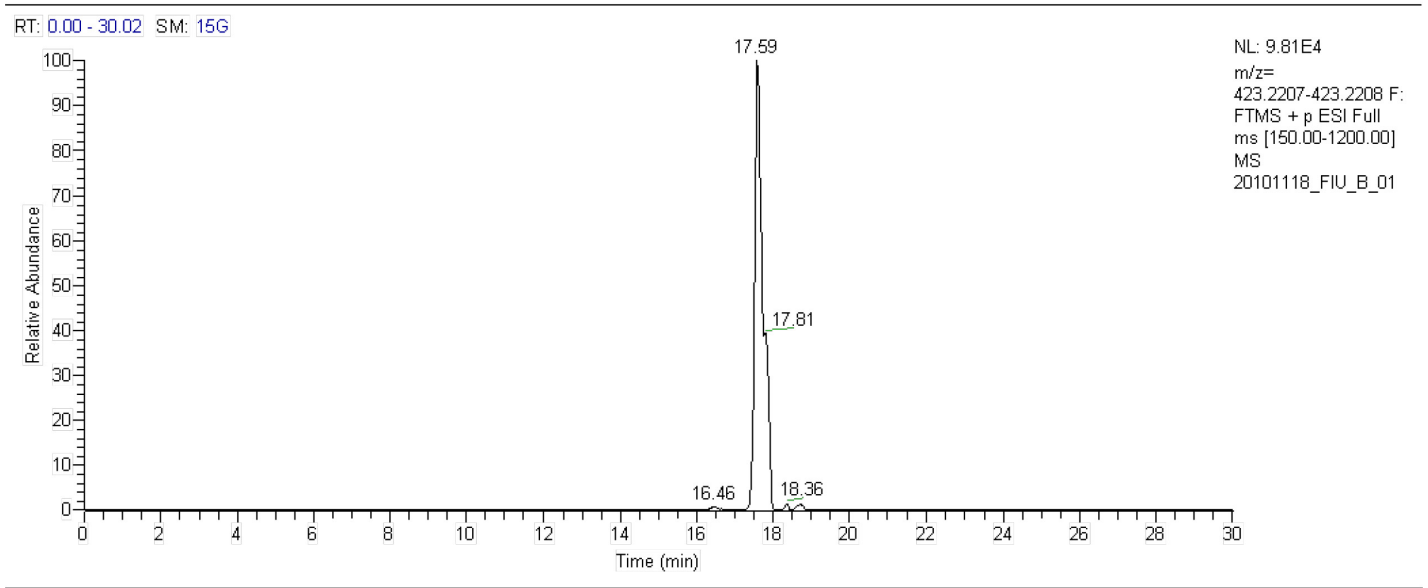

20101118_FIU_B_01 \#3234-3267 RT: 17.72-17.83 AV: 6 NL: 1.93 E5

F: FTMS + p ESIFull ms [150.00-1200.00]

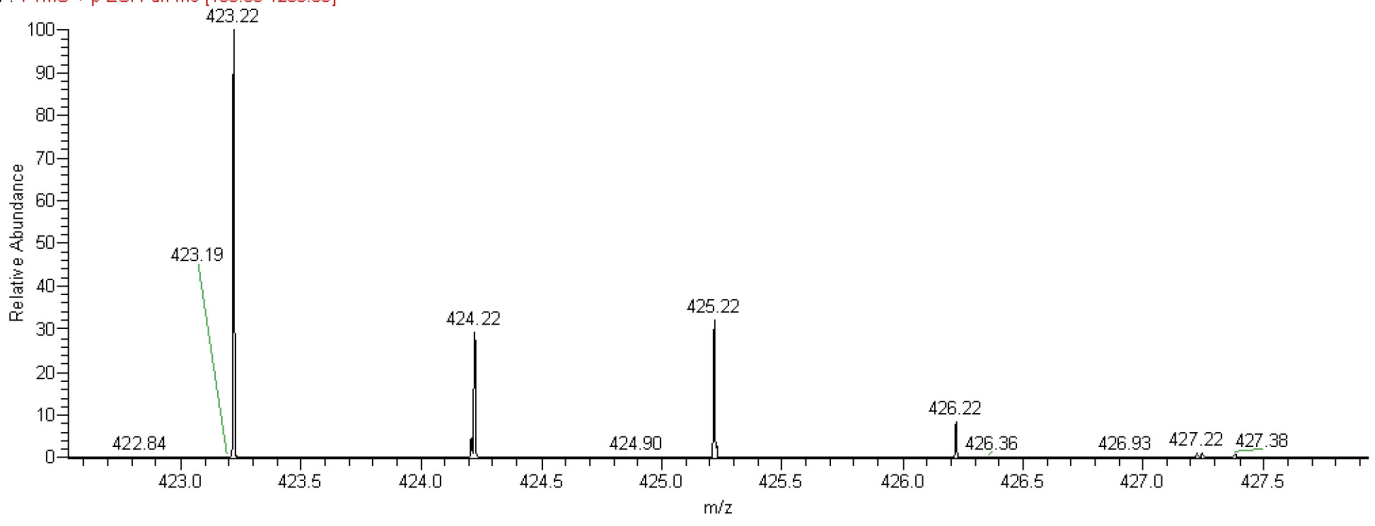

Figure 40: Selection of 2 from the OT Velos HRHESIMS Data 


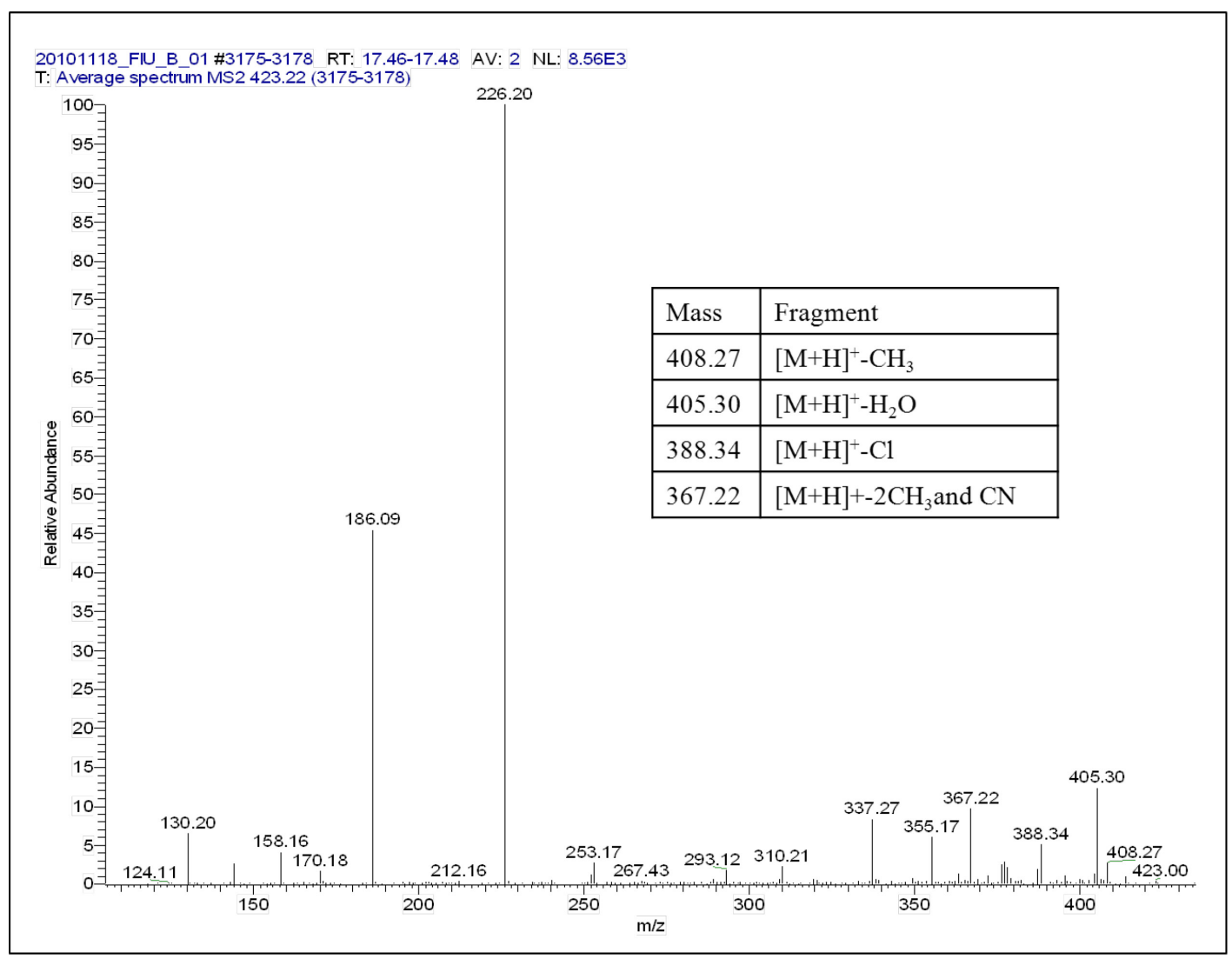

Figure 41: HRHESIMS MS/MS of 2

From the mass spec data it was suggested that $\mathbf{2}$ was Ambiguine B Isonitrile, but further analysis with IR suggested that conclusion was incorrect. From the IR Spectrum (Figure 42) it was observed that 2 lacked the peak at $2135 \mathrm{~cm}-1$ that is produced by the isonitrile group (Smitka et al., 1992). It may be possible that as a result of the small amounts used to analyze $\mathbf{2}$ with the IR, the spectrum was not a true representation of functional groups in the compound. Even if this was not the case, the lack of an isonitrile group was also observed in the carbon NMR of 2 (discussed later in this chapter). 


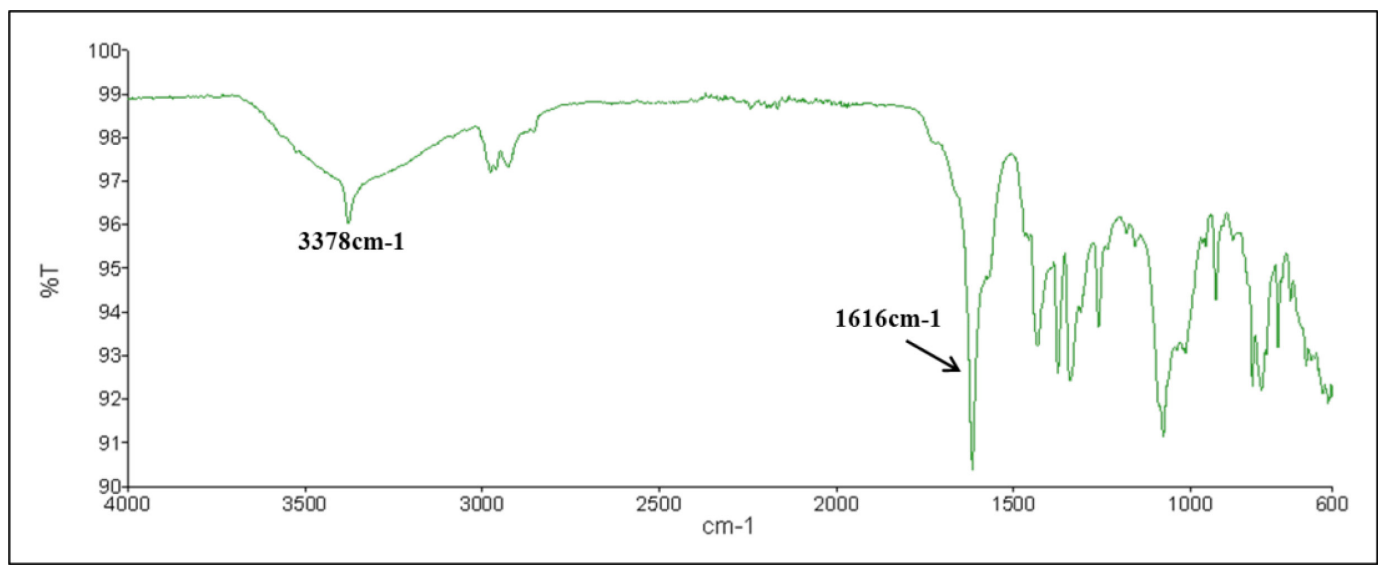

Figure 42: IR spectrum of 2

As with 12-epi-Hapalindole H Isonitrile, three sets of purification of the ECS was required to obtain enough of 2 to allow ${ }^{13} \mathrm{C}$ NMR analysis. Proton NMR in MeOD was originally performed, but it was later determined that $\mathrm{C}_{6} \mathrm{D}_{6}$ was a superior solvent for this molecule. The $\mathrm{C}_{6} \mathrm{D}_{6}$ allowed a larger amount of $\mathbf{2}$ to de dissolved and prevented the contamination of less soluble impurities. Using $\mathrm{C}_{6} \mathrm{D}_{6},{ }^{1} \mathrm{H} \mathrm{NMR}, \mathrm{COSY},{ }^{13} \mathrm{C} \mathrm{NMR}$, DEPT, HMQC and NOESY were performed (Figures 43-48). 


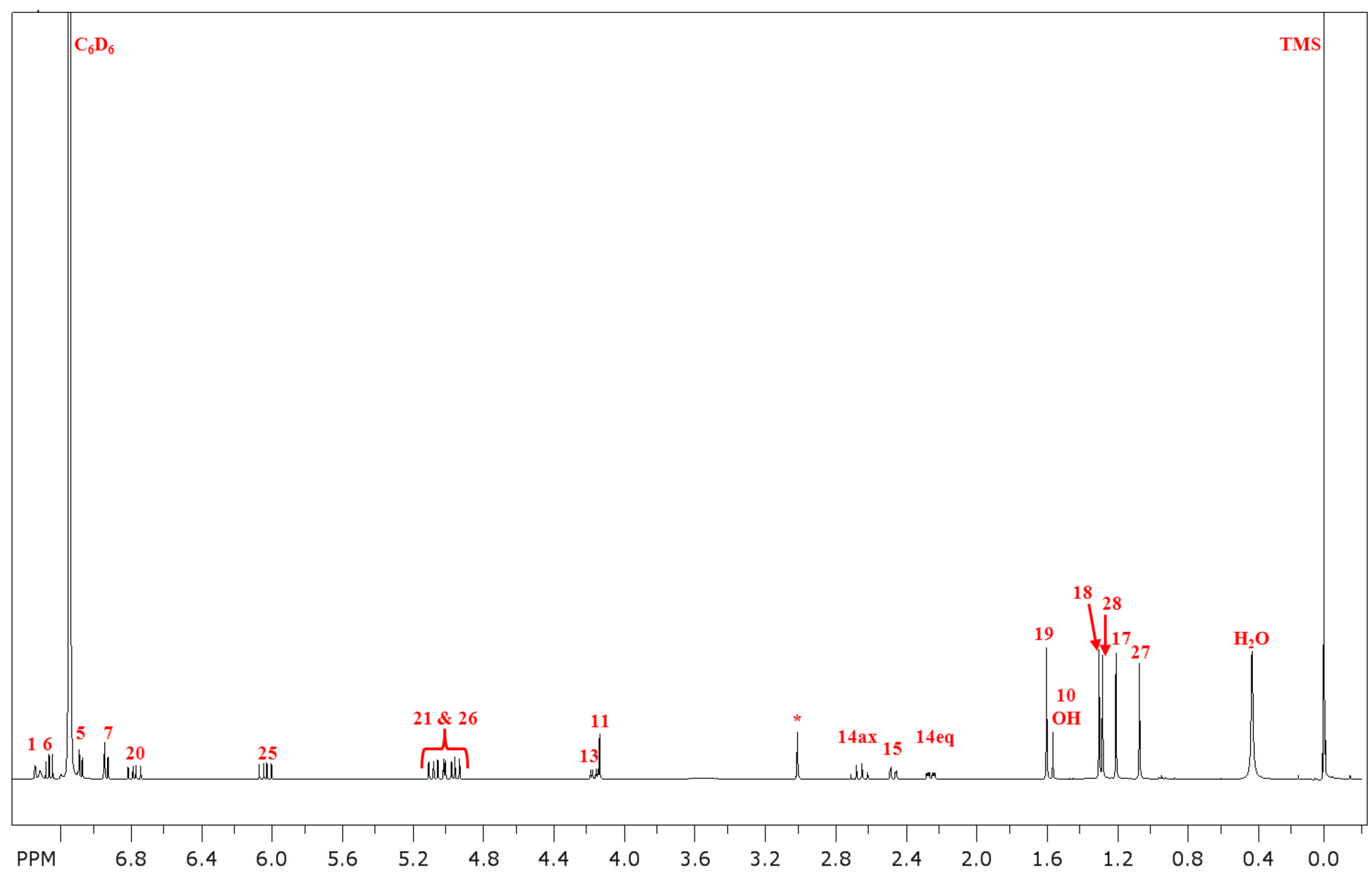

Figure 43: ${ }^{H}$ NMR of Compound 2 in $C_{6} D_{6}$ (* Denotes chemical shifts produced by impurities.) 


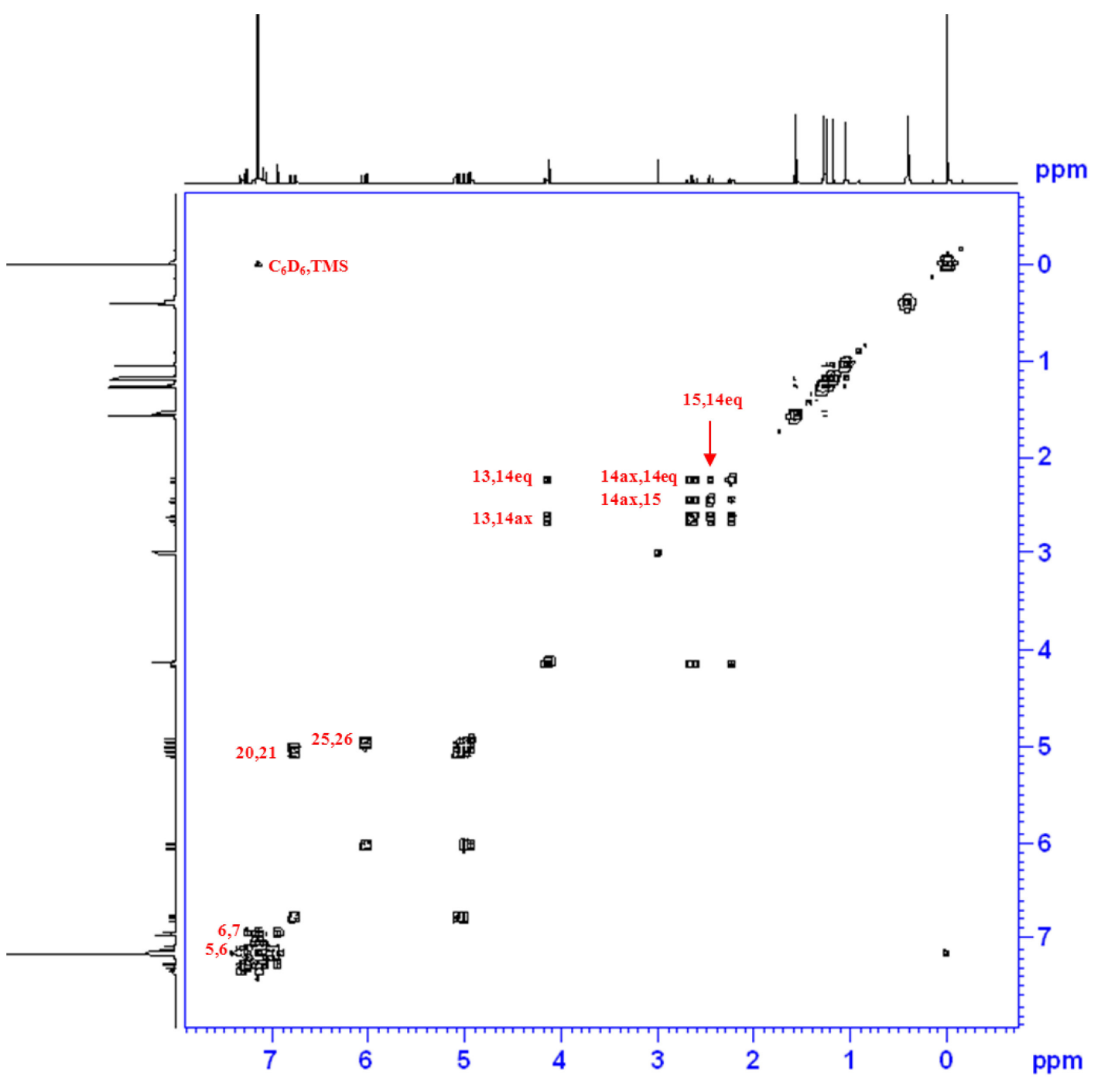

Figure 44:COSY in $C_{6} D_{6}$ of 2 


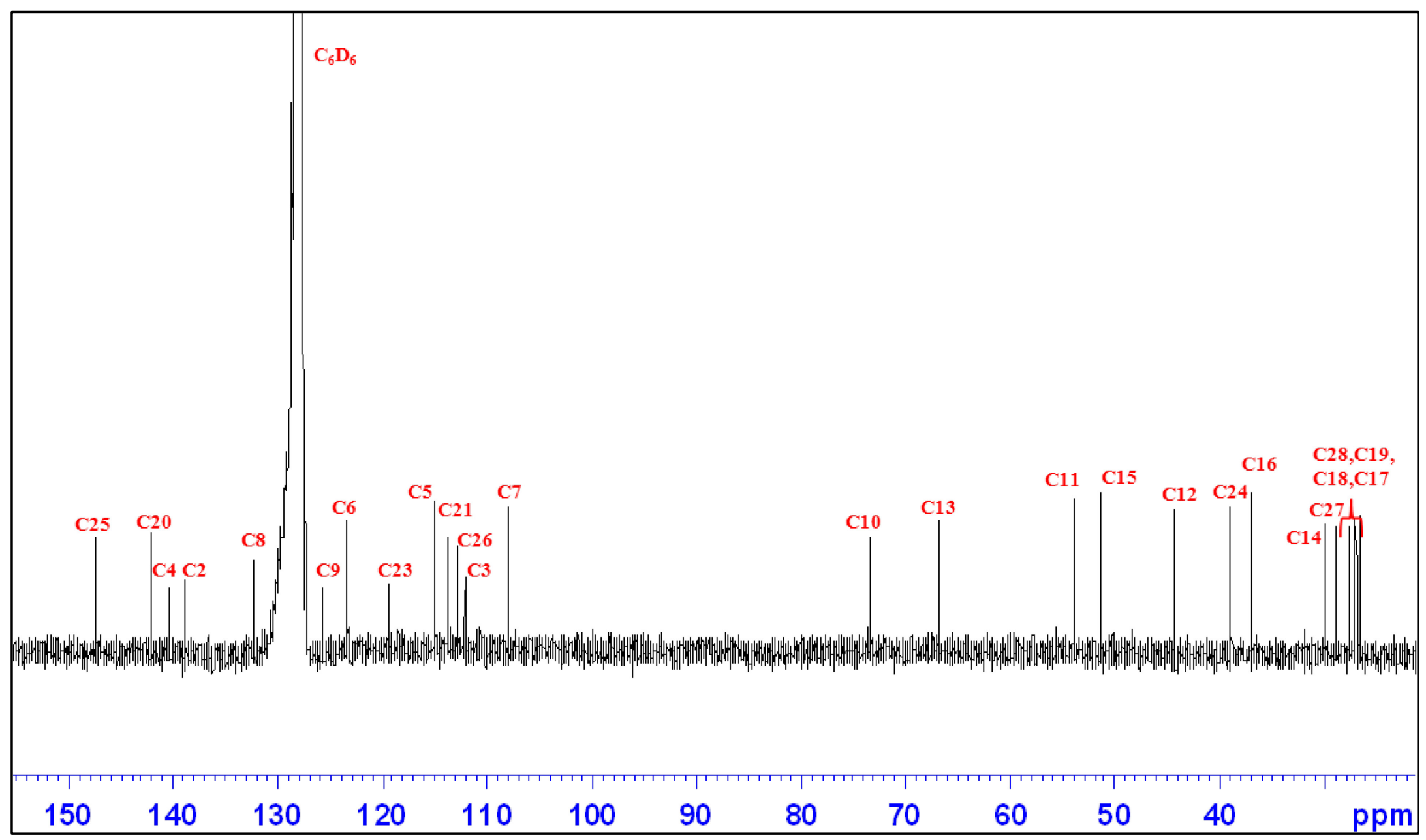

Figure $45:{ }^{13} C$ NMR of 2 in $C_{6} D_{6}$ 


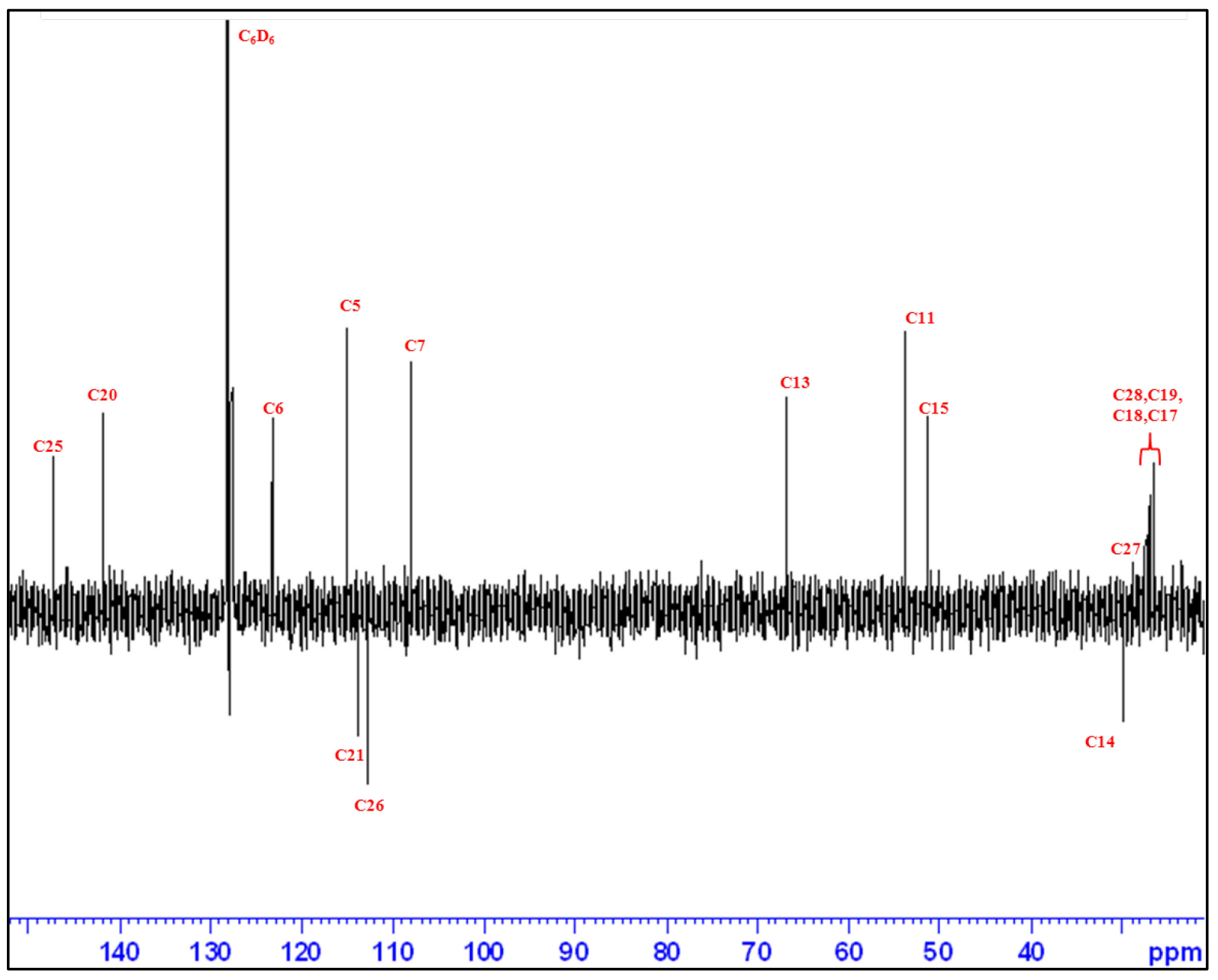

Figure 46: DEPT in $C_{6} D_{6}$ of 2 


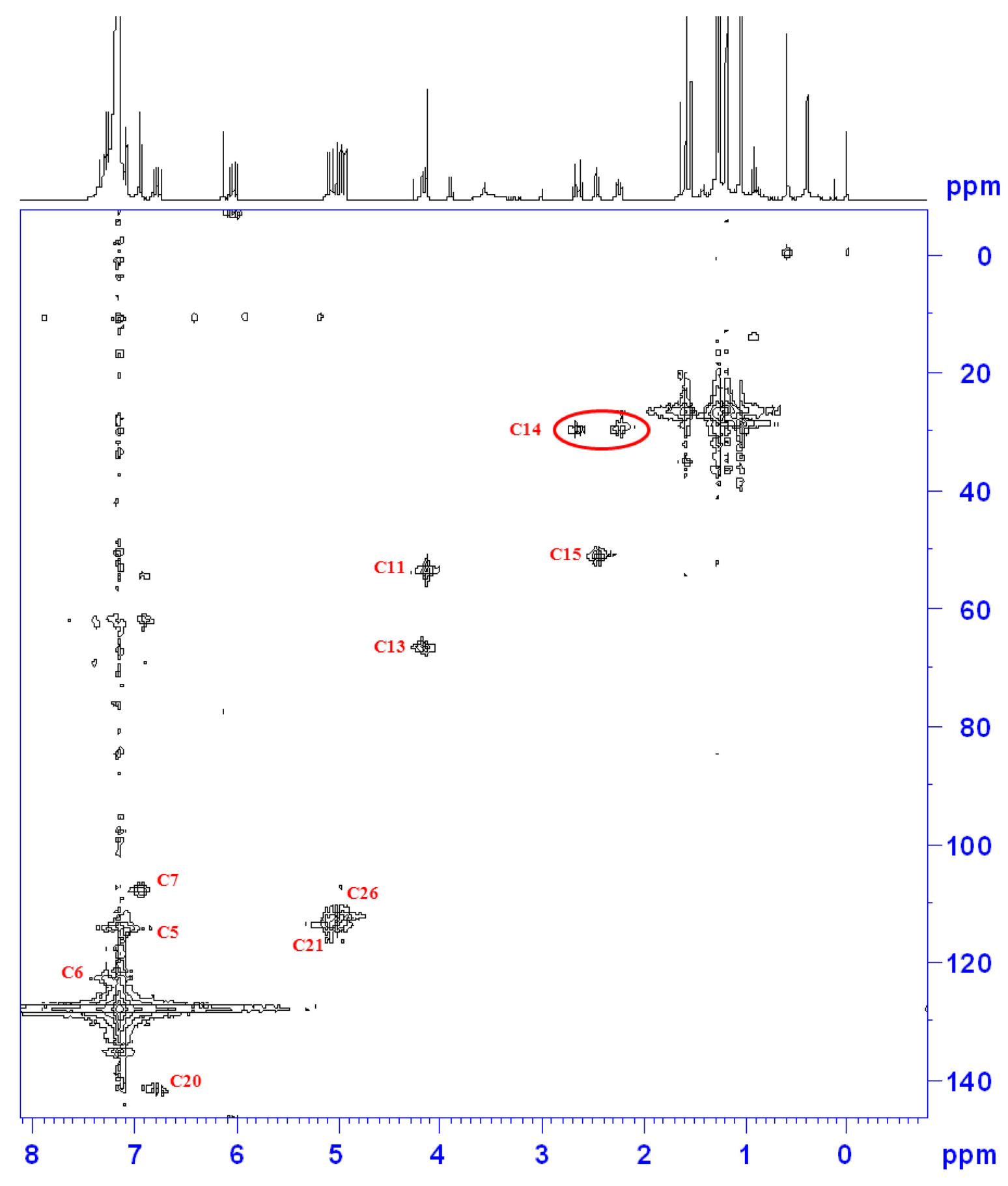

Figure 47: HMQC in $C_{6} D_{6}$ of 2 


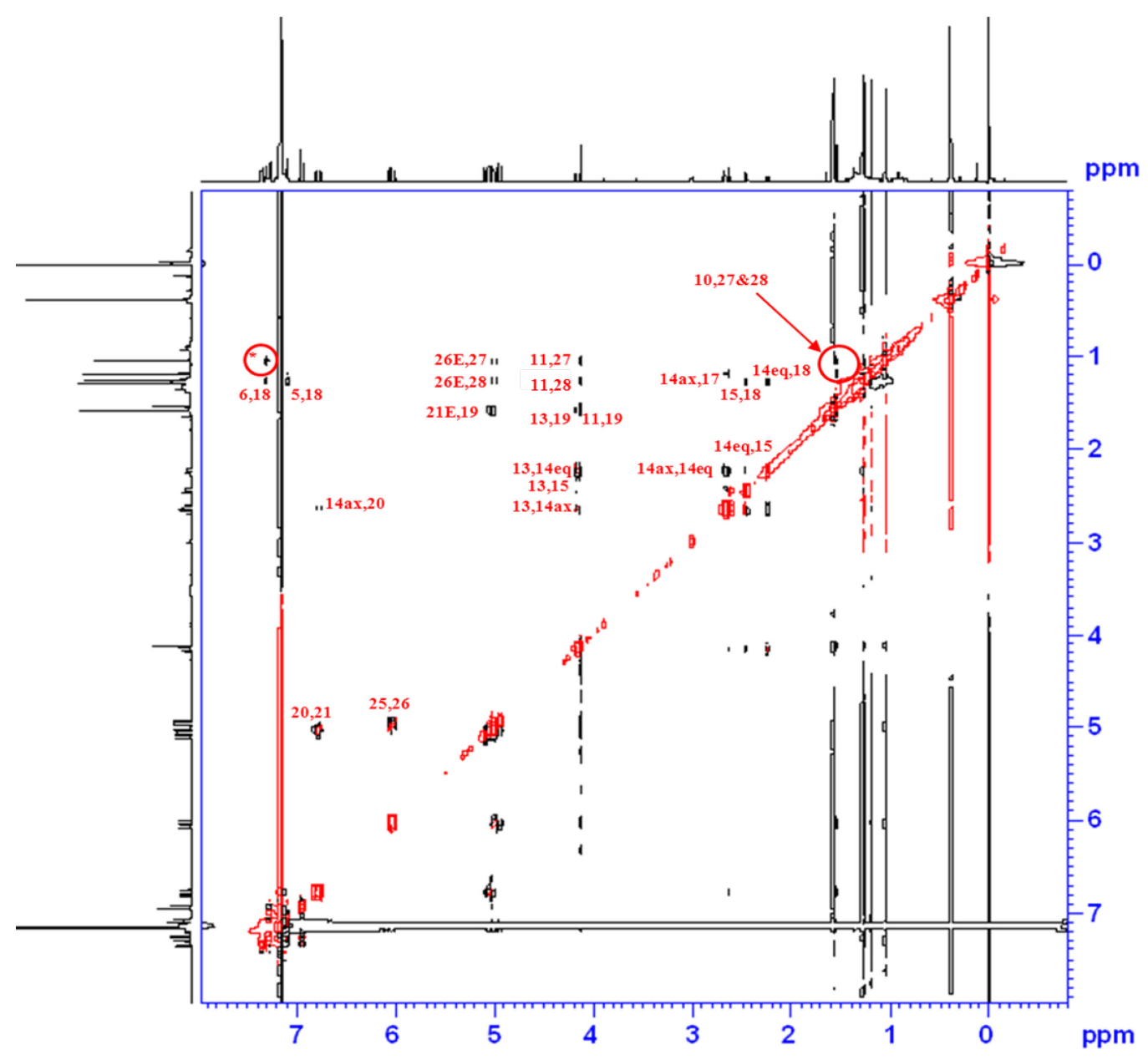

Figure 48:NOESY in $C_{6} D_{6}$ of 2. ("Denotes unassigned correlation.)

The ${ }^{1} \mathrm{H}$ NMR revealed that 2 contained 32 hydrogens which contradicted the formula proposed by the mass spectrometric analysis. The singlet at $3.0 \delta$ was not shown to correlate with any other hydrogen in the COSY nor any carbon in the HMQC. The sample was dried and stored in the freezer before the NOESY was performed; upon analysis it was revealed that the singlet disappeared altogether. It was because of these observations that the singlet was suggested to be an impurity. With the singlet removed from the analysis, the splitting patterns and the chemical shifts of $\mathbf{2}$ were similar to those produced by the protons in Ambiguine B Isonitrile as shown in Table 9. It is clear from Table 9 that the chemical shifts of many of the protons are different from those of 
Ambiguine B Isonitrile. The dissimilarity observed in the proton chemical shifts may be a result of the difference in solvents used. The ${ }^{1} \mathrm{H}$ NMR revealed functional groups in the structure that were also observed for Ambiguine B Isonitrile. These groups included; 2 terminal alkenes, 5 methyl groups, an indole moiety bonded at the $\mathrm{C} 2, \mathrm{C} 3$, and $\mathrm{C} 4$ carbons, and a cyclohexane ring. All of these observations suggested that 2 was Ambiguine B Isonitrile, but the ${ }^{13} \mathrm{C}$ NMR and IR disagreed with this assignment.

Table 9: Proton Chemical Shifts from ${ }^{1} \mathrm{H}$ NMR of 2 and Ambiguine B Isonitrile (400 $\mathrm{MHz}, \mathrm{J}$ in $\mathrm{Hz}$ ) * A quartet splitting pattern was observed, but due to the difference of the chemical environments of the coupling hydrogens, it was suggested the splitting pattern was an overlapping doublet of doublet of doublets.

\begin{tabular}{|c|c|c|}
\hline $\begin{array}{c}\text { Proton } \\
\text { Position }\end{array}$ & $\begin{array}{c}\text { Compound } \\
\mathbf{2} \\
\left(\mathbf{C}_{6} \mathbf{D}_{6}\right)\end{array}$ & $\begin{array}{c}\text { Ambiguine } \mathbf{B} \\
\text { Isonitrile } \\
\text { (CHCl3) } \\
\text { Smitka et al., } \mathbf{1 9 9 2}\end{array}$ \\
\hline $\mathbf{1}$ & $7.32 \mathrm{~s}$ & $8.18 \mathrm{br} \mathrm{s}$ \\
\hline $\mathbf{5}$ & $7.10 \mathrm{dd}(0.5,7.3)$ & $7.07 \mathrm{dd}(0.8,7.3)$ \\
\hline $\mathbf{6}$ & $7.27 \mathrm{dd}(7.3,8)$ & $7.17 \mathrm{~m}(7.3,8.1)$ \\
\hline 7 & $6.96 \mathrm{dd}(0.5,8)$ & $7.15 \mathrm{~m}(0.8,8.1)$ \\
\hline $\mathbf{1 1} \mathbf{~ e q}$ & $4.13 \mathrm{~s}$ & $4.68 \mathrm{~s}$ \\
\hline $\mathbf{1 3} \mathbf{~ a x}$ & $4.16 \mathrm{dd}(4.4,12.6)$ & $4.41 \mathrm{dd}(4.1,12.2)$ \\
\hline $\mathbf{1 4} \mathbf{~ a x}$ & $2.65 \mathrm{q} *(12.5)$ & $2.53 \mathrm{q}(12.7)$ \\
\hline $\mathbf{1 4} \mathbf{~ e q}$ & $2.25 \mathrm{ddd}(2.2,4.3,-13)$ & $2.30 \mathrm{ddd}(2.3,4.1,-13.1)$ \\
\hline $\mathbf{1 5} \mathbf{~ a x}$ & $2.45 \mathrm{dd}(2,12.6)$ & $2.41 \mathrm{ddd}(2.3,12.7)$ \\
\hline $\mathbf{1 7}$ & $1.18 \mathrm{~s}$ & $1.23 \mathrm{~s}$ \\
\hline $\mathbf{1 8}$ & $1.28 \mathrm{~s}$ & $1.54 \mathrm{~s}$ \\
\hline $\mathbf{1 9}$ & $1.58 \mathrm{~s}$ & $1.53 \mathrm{~s}$ \\
\hline $\mathbf{2 0}$ & $6.78 \mathrm{dd}(11,17.6)$ & $6.04 \mathrm{dd}(10.9,17.6)$ \\
\hline $\mathbf{2 1 \mathbf { E }}$ & $5.05 \mathrm{dd}(1,17.6)$ & $5.31 \mathrm{~d}(17.6)$ \\
\hline $\mathbf{2 1} \mathbf{Z}$ & $5.09 \mathrm{dd}(1,11)$ & $5.26 \mathrm{~d}(10.9)$ \\
\hline $\mathbf{2 5}$ & $6.05 \mathrm{dd}(10.5,17.6)$ & $6.33 \mathrm{dd}(10.4,17.6)$ \\
\hline $\mathbf{2 6 E}$ & $5.00 \mathrm{dd}(1,17.6)$ & $5.28 \mathrm{~d}(17.6)$ \\
\hline $\mathbf{2 6 \mathbf { Z }}$ & $4.95 \mathrm{dd}(1,10.5)$ & $5.36 \mathrm{~d}(10.4)$ \\
\hline $\mathbf{2 7}$ & $1.05 \mathrm{~s}$ & $1.62 \mathrm{~s}$ \\
\hline $\mathbf{2 8}$ & $1.26 \mathrm{~s}$ & $1.64 \mathrm{~s}$ \\
\hline $\mathbf{1 0 - O H}$ & $1.54 \mathrm{~d}(0.5)$ & $1.67 \mathrm{~s}$ \\
\hline
\end{tabular}


The ${ }^{13} \mathrm{C}$ NMR revealed 26 carbons which corroborates the formula produced by the mass spectrometric analysis. However, the carbon that should have produced the chemical shift at $159 \delta$ was not observed thereby confirming the lack of the isonitrile group. Table 10 depicts the comparison of $\mathbf{2}$ with Ambiguine B Isonitrile. The assignment of the carbons was done by analyzing the HMQC, DEPT, and NOESY. Proton chemical shifts were assigned to a carbon with the HMQC and the carbons were numbered on the basis of the comparison of the ${ }^{1} \mathrm{H}$ NMR data of $\mathbf{2}$ and Ambiguine B Isonitrile. Carbons without protons were assigned by comparing the closest chemical shifts to those reported for Ambiguine B Isonitrile. The carbons were numbered in the same fashion of many of the hapalindole-like indole alkaloids, especially the ambiguines (Figure 23) (Smitka et al., 1992). When examining the two sets of data it is apparent that the compounds differ in one region, which is observed with the difference in the values of $\mathrm{C} 11, \mathrm{C} 19$, and C23 from those published for Ambiguine B Isonitrile. It was from this observation, and the IR, that $\mathbf{2}$ was determined to be a new ambiguine variant. 
Table 10: Carbon Chemical Shifts from ${ }^{13}$ C NMR of 2 and Ambiguine B Isonitrile

\begin{tabular}{|c|c|c|}
\hline $\begin{array}{l}\text { Carbon } \\
\text { Position }\end{array}$ & $\begin{array}{c}\text { Compound } \\
2 \\
\left(\mathrm{C}_{6} \mathrm{D}_{6}\right)\end{array}$ & $\begin{array}{c}\text { Ambiguine B } \\
\text { Isonitrile } \\
\text { (CHCl3) } \\
\text { Smitka et al., } 1992\end{array}$ \\
\hline 2 & 139.5 & 138.8 \\
\hline 3 & 113.4 & 111.4 \\
\hline 4 & 140.9 & 139.8 \\
\hline 5 & 115.6 & 114.4 \\
\hline 6 & 123.9 & 122.9 \\
\hline 7 & 108.7 & 107.6 \\
\hline 8 & 132.9 & 131.8 \\
\hline 9 & 126.3 & 125.4 \\
\hline 10 & 73.9 & 73.8 \\
\hline 11 & 54.4 & 68.5 \\
\hline 12 & 44.9 & 45.3 \\
\hline 13 & 67.4 & 63.9 \\
\hline 14 & 30.5 & 29.1 \\
\hline 15 & 52.0 & 48 \\
\hline 16 & 37.6 & 36.7 \\
\hline 17 & 27.2 & 26.5 \\
\hline 18 & 27.7 & 27.1 \\
\hline 19 & 27.6 & 18.5 \\
\hline 20 & 142.7 & 144.2 \\
\hline 21 & 114.5 & 115.5 \\
\hline 23 & 120.1 & 159.0 \\
\hline 24 & 39.5 & 39 \\
\hline 25 & 148.0 & 146.8 \\
\hline 26 & 113.3 & 112.7 \\
\hline 27 & 29.4 & 28.9 \\
\hline 28 & 28.1 & 27.6 \\
\hline
\end{tabular}

The structure of $\mathbf{2}$ was deduced by first assigning protons to determine similarities to Ambiguine B Isonitrile. In the proton NMR, a similar splitting of proton chemical shifts produced by the benzoid ring of the indole moiety was observed as with $\mathbf{1}$, however, the signal produced by the proton at C2 was missing (Figure 49). The doublet 
of doublet at $7.27 \delta$ was shown to couple to the doublets of doublets at $7.10 \delta$ and $6.96 \delta$ with coupling constants equal to the ${ }^{3} \mathrm{~J}_{5,6}$ and ${ }^{3} \mathrm{~J}_{6,7}$ observed for $\mathbf{1}$ in the previous section. From the ${ }^{1} \mathrm{H}$ NMR data it can be deduced that the indole moiety was substituted at the $\mathrm{C} 4, \mathrm{C} 3$, and $\mathrm{C} 2$ positions (Figure 50). The doublet of doublets at $7.27 \delta$ was assigned as the proton on the C6 carbon and the corresponding carbon at $123.9 \delta$ was assigned as C6. The two remaining doublets of doublets were assigned on the basis of the carbon correlations observed in the HMQC. The doublet of doublets at $7.10 \delta$ correlated to the carbon with the chemical shift of $115.6 \delta$. The value of the carbon chemical shift and the doublet of doublets in the aromatic region matched the C5 carbon. The doublet of doublets at $6.96 \delta$ was shown to correlate to a carbon with the chemical shift of $108.7 \delta$, as with $\mathrm{C} 5$, the chemical shift of the carbon and proton allowed the assignment of the $\mathrm{C} 7$ carbon. The lack of the $\mathrm{C} 2$ proton agreed with the structure of previously identified ambiguines, which have an isoprenyl unit attached at that position. 


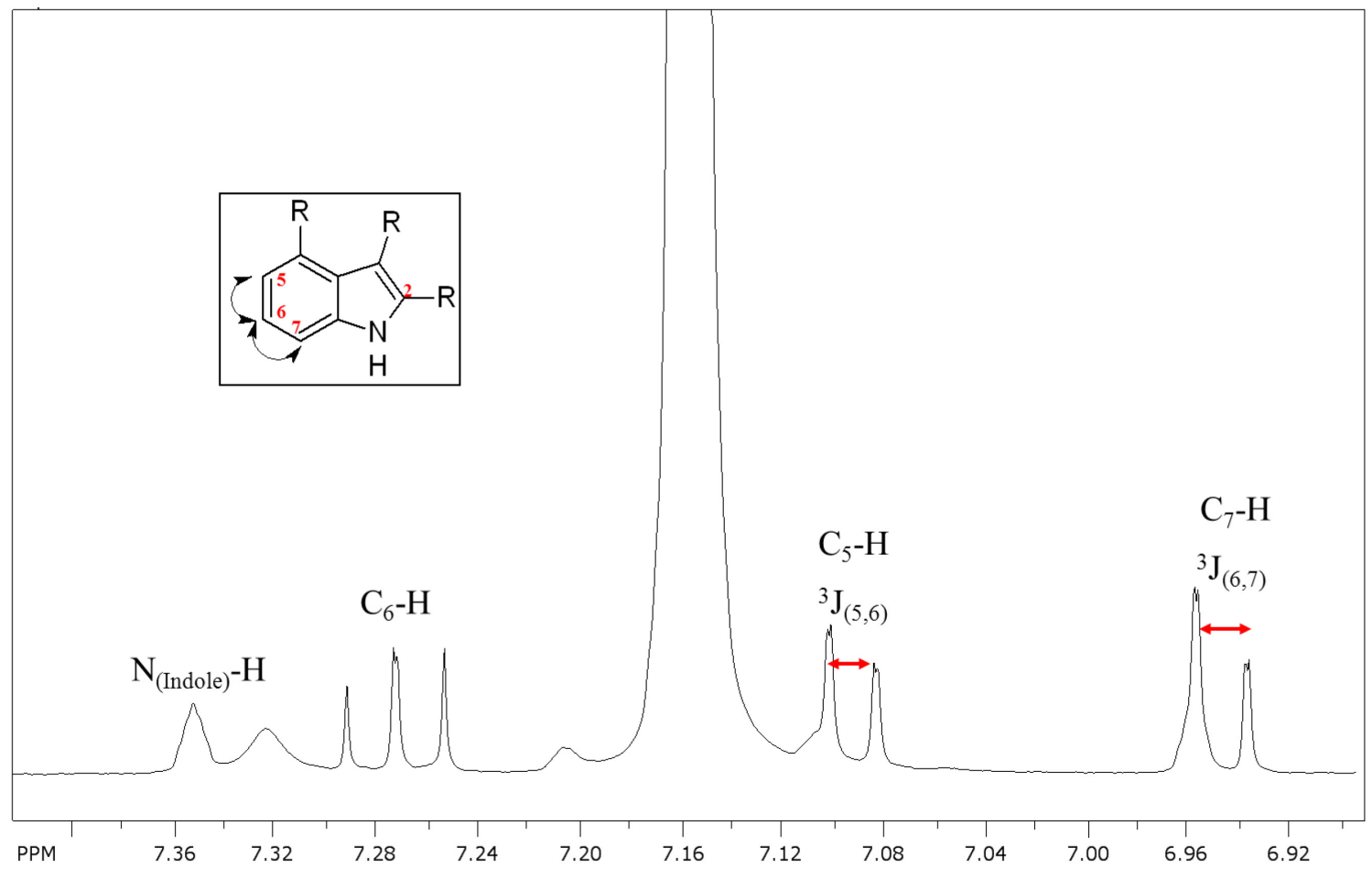

Figure 49: Aromatic Region of $1 \mathrm{H}$ NMR of 2

In the unsaturated region of the ${ }^{1} \mathrm{H}$ NMR it was observed that two terminal alkenes occurred in $\mathbf{2}$ (Figures 50 \& 51). The assignment of two terminal alkenes for the six sets of doublets of doublets was determined by analyzing the COSY and HMQC. Each of the six sets of doublets of doublets was shown to integrate to one hydrogen. The doublets of doublets at $5.05 \delta$ and $5.09 \delta$ were shown to couple to the doublet of doublets at $6.78 \delta$ with coupling constants of 11 and $17.6 \mathrm{~Hz}$. Of the doublet of doublets at 5.05 and $5.09 \delta$ the $\mathrm{E}$ and $\mathrm{Z}$ protons were determined on the basis of the value of the coupling constants. The $\mathrm{Z}$ position was assigned as the doublet of doublets at $5.09 \delta$ with the smaller coupling constant (Pavia et al., 2009). The E position proton was then assigned as the remaining proton with the higher coupling constant (Pavia et al., 2009). The remaining doublet of doublets at $6.05 \delta$ was shown to correlate to the other two doublets 
of doublets at 5.00 and $4.95 \delta$. The coupling constants were again used to assign the $\mathrm{E}$ and $\mathrm{Z}$ protons. The $\mathrm{E}$ proton was assigned to the doublet of doublets at $5.00 \delta$ with a coupling constant of $17.6 \mathrm{~Hz}$ and the $\mathrm{Z}$ proton was assigned as the remaining doublet of doublets at $4.95 \delta$ with a coupling constant of $10.5 \mathrm{~Hz}$.

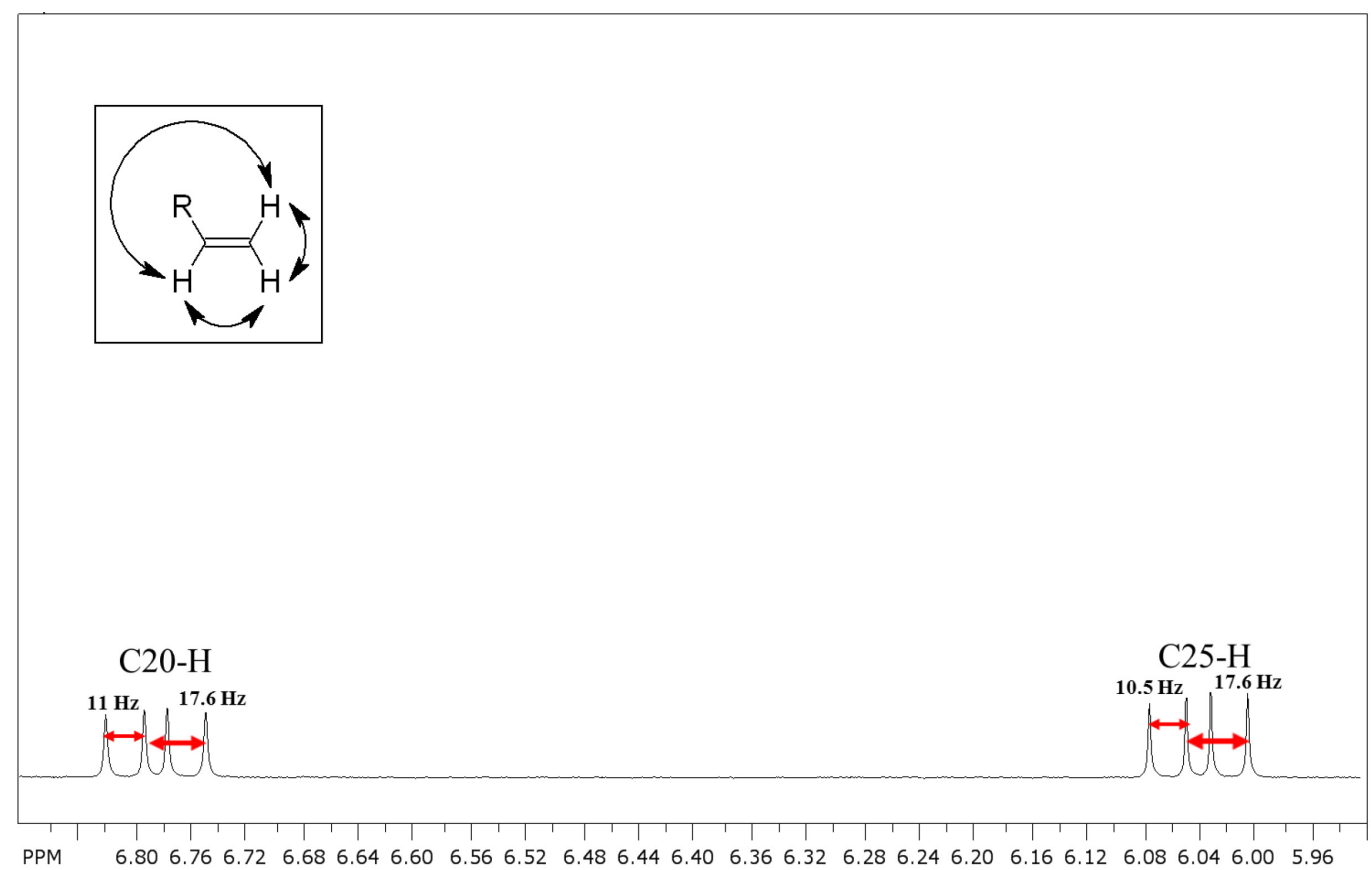

Figure 50: Unsaturated Region of $1 \mathrm{H}$ NMR for 2 6-7ppm 


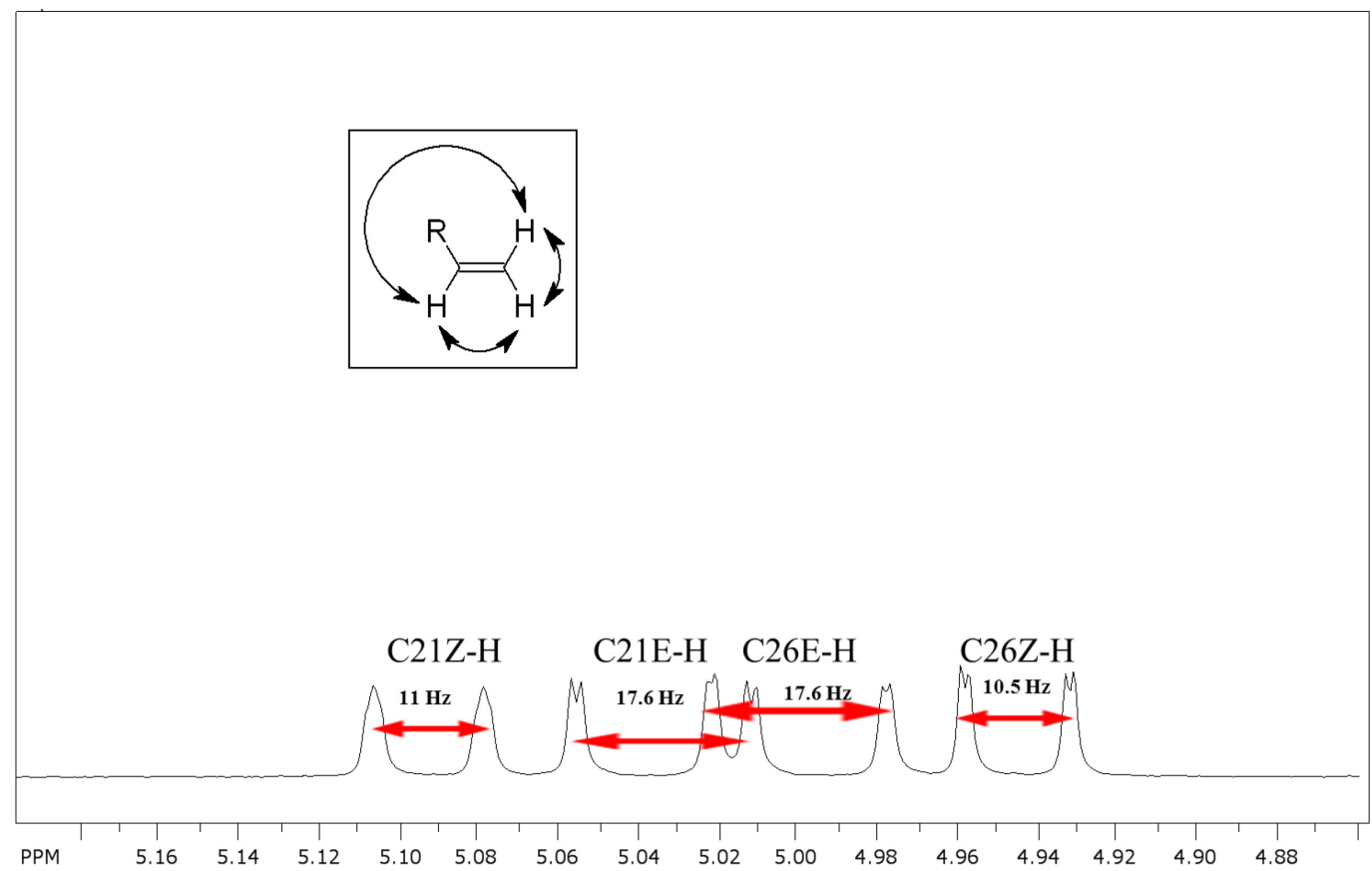

Figure 51: Unsaturated Region of 1H NMR for 2 4.8-5.2ppm

A cyclohexane component was deduced in the structure of $\mathbf{2}$ with the splitting patterns and coupling constants of the protons at 4.16, 4.13, 2.65, 2.25, and $2.45 \delta$, each of which integrated to one hydrogen (Figures 52 \& 53). The doublet of doublets at $4.16 \delta$ was deduced to be attached to a carbon with a halogen atom attached on the basis of the chemical shift. The assignment of a proton with that chemical shift correlates to the chlorine isotope pattern observed in the mass spectrometric analysis. The doublet of doublets was integrated to one hydrogen, and was shown to be coupled to the quartet and the doublet of doublets of doublets at $2.25 \delta$. The quartet and the doublet of doublets of

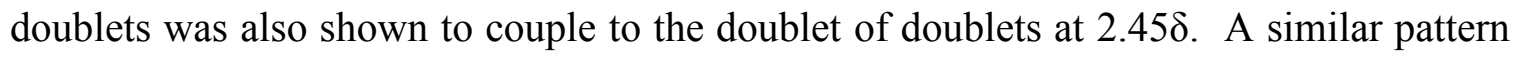
was observed with the data for Ambiguine B Isonitrile (Table 9). The coupling constant observed in the proton splitting was also very similar to that observed by Smitka et al., (1992) for Ambiguine B Isonitrile. The coupling constants calculated for the doublet of doublets at $4.16 \delta$ were 4.4 and $12.6 \mathrm{~Hz}$. The calculated coupling constants matches the 
coupling constants for the coupling of C13 and C14ax which was $12.5 \mathrm{~Hz}$ and C13 and C14eq which was $4.4 \mathrm{~Hz}$ in the Ambiguine B cyclohexane ring. The similar chemical shift, splitting pattern, and coupling constants suggested a cyclohexane ring in the structure, and the assignment of the doublet of doublets at $4.16 \delta$ as the proton on $\mathrm{C} 13$. The carbon correlated to the doublet of doublets was determined by analyzing the HMQC data, and showed a correlation to a carbon chemical shift at $67.4 \delta$ which was comparable to the $\mathrm{C} 13$ carbon of Ambiguine B Isonitrile.

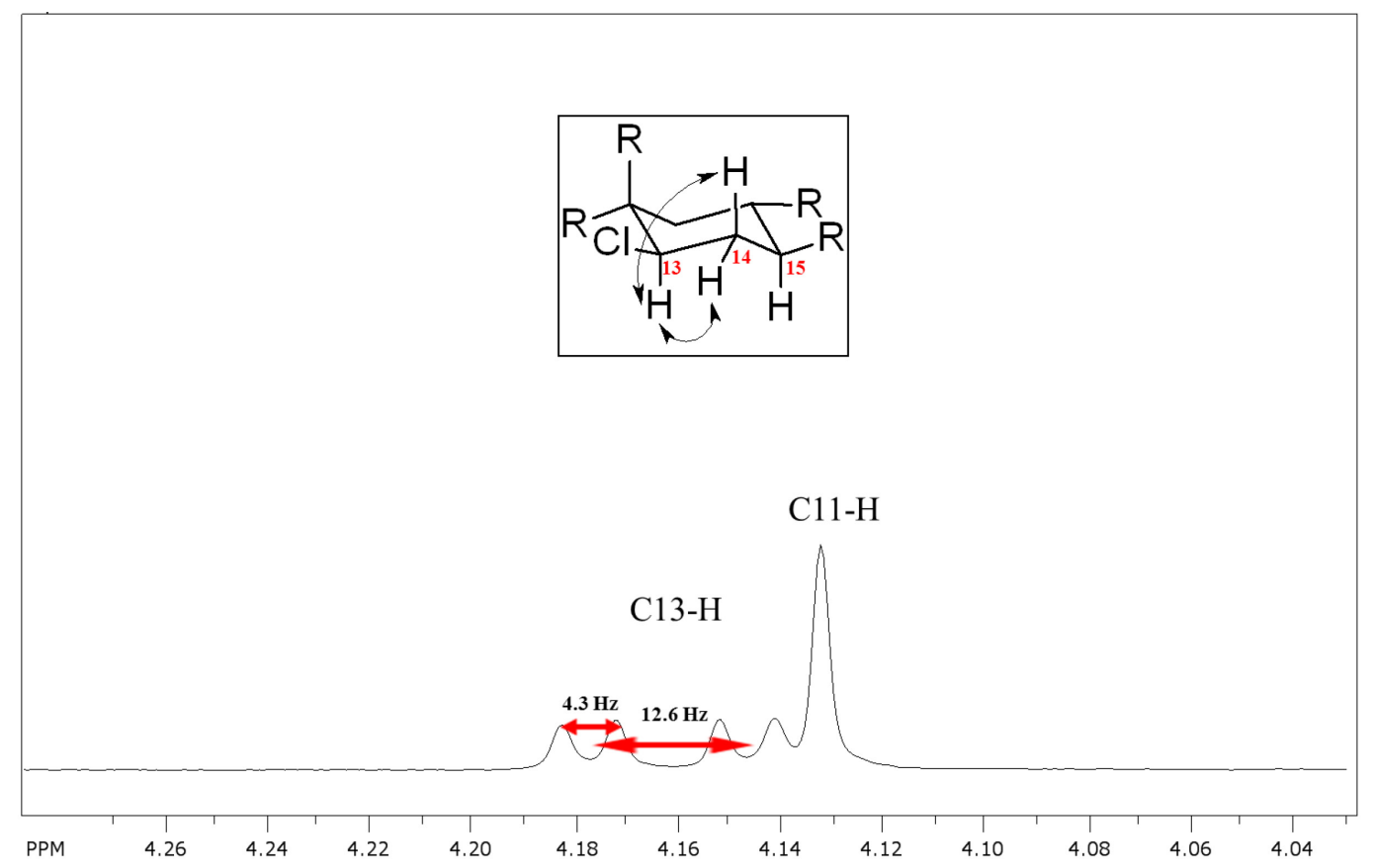

Figure 52: 1 H NMR of 2 4-4.3ppm 


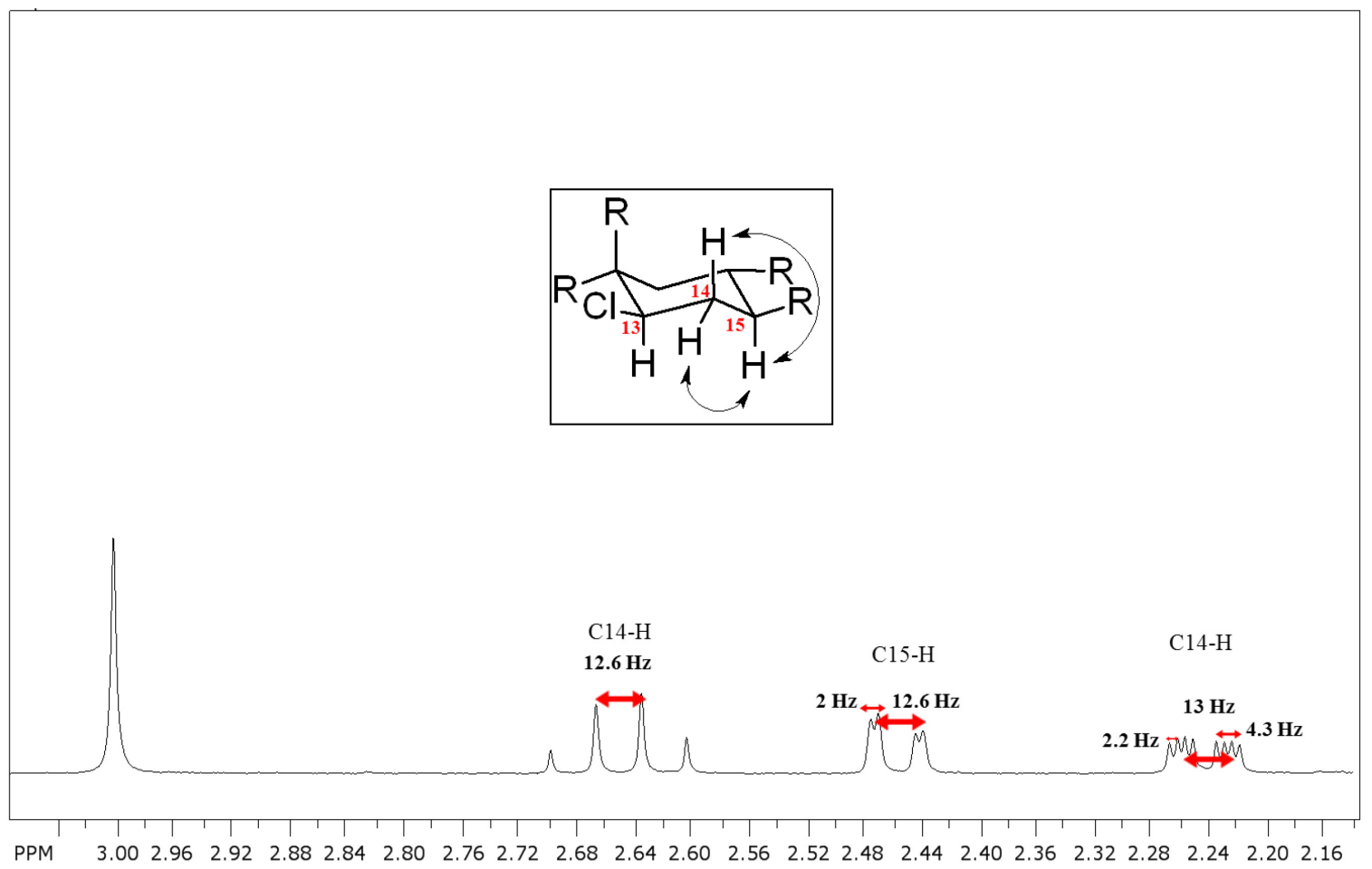

Figure 53: $1 \mathrm{H}$ NMR of 2 2.2-3.2ppm

The quartet at $2.65 \delta$ was assigned as the axial proton on $\mathrm{C} 14$ on the basis of the chemical shift and splitting pattern observed with Ambiguine B Isonitrile (Smitka et al., 1992). The doublet of doublets of doublets (ddd) at $2.25 \delta$ was assigned as the equatorial proton on $\mathrm{C} 14$ on the basis of similar splitting patterns and coupling constants. The coupling constants calculated for the ddd at $2.25 \delta$ were $2.2,4.3$, and $13 \mathrm{~Hz}$, which matches the values observed by Smitka et al., (1992). Smitka observed the coupling of $14 \mathrm{eq}$ and 13 protons, which was previously shown to be $4.4 \mathrm{~Hz}$. The coupling of the $14 \mathrm{eq}$ and 14ax protons was $-13 \mathrm{~Hz}$, and the $14 \mathrm{eq}$ and 15 protons was $2.8 \mathrm{~Hz}$. Given the comparable coupling constants and splitting patterns the ddd at $2.25 \delta$ was assigned as the equatorial proton on $\mathrm{C} 14$. The quartet and ddd were shown to correlate to the same carbon at $30.5 \delta$ which had a comparable chemical shift to C14. 
The doublet of doublets at 2.45 was shown to couple to both protons on C14 and was suggested to be the proton on C15. The coupling constant calculated by Smitka et al., (1992) for the coupling of 15 to $14 \mathrm{ax}$ was $12.5 \mathrm{~Hz}$ and 15 to $14 \mathrm{eq}$ was $2.8 \mathrm{~Hz}$. The coupling constants calculated for the doublet of doublets were 2 and $12.6 \mathrm{~Hz}$ which matches the values produced by Smitka et al., (1992). The doublet of doublets at $2.45 \delta$ was then assigned as the proton on $\mathrm{C} 15$. As a result of the $12.6 \mathrm{~Hz}$ coupling constant with 14 ax and the $2 \mathrm{~Hz}$ with the $14 \mathrm{eq}$ proton, it was determined that $\mathrm{C} 15$ was axial. The coupling of the $\mathrm{C} 13, \mathrm{C} 14$, and $\mathrm{C} 15$ protons was confirmed with the COSY experiments observed in Figure 54. The singlet at $4.13 \delta$ had a comparable chemical shift to the proton on $\mathrm{C} 11$ which also produced a singlet as a result of the lack of protons on any of the adjacent carbons. In Ambiguine B Isonitrile C11 is isolated from the protons in the cyclohexane ring and would produce a singlet. 


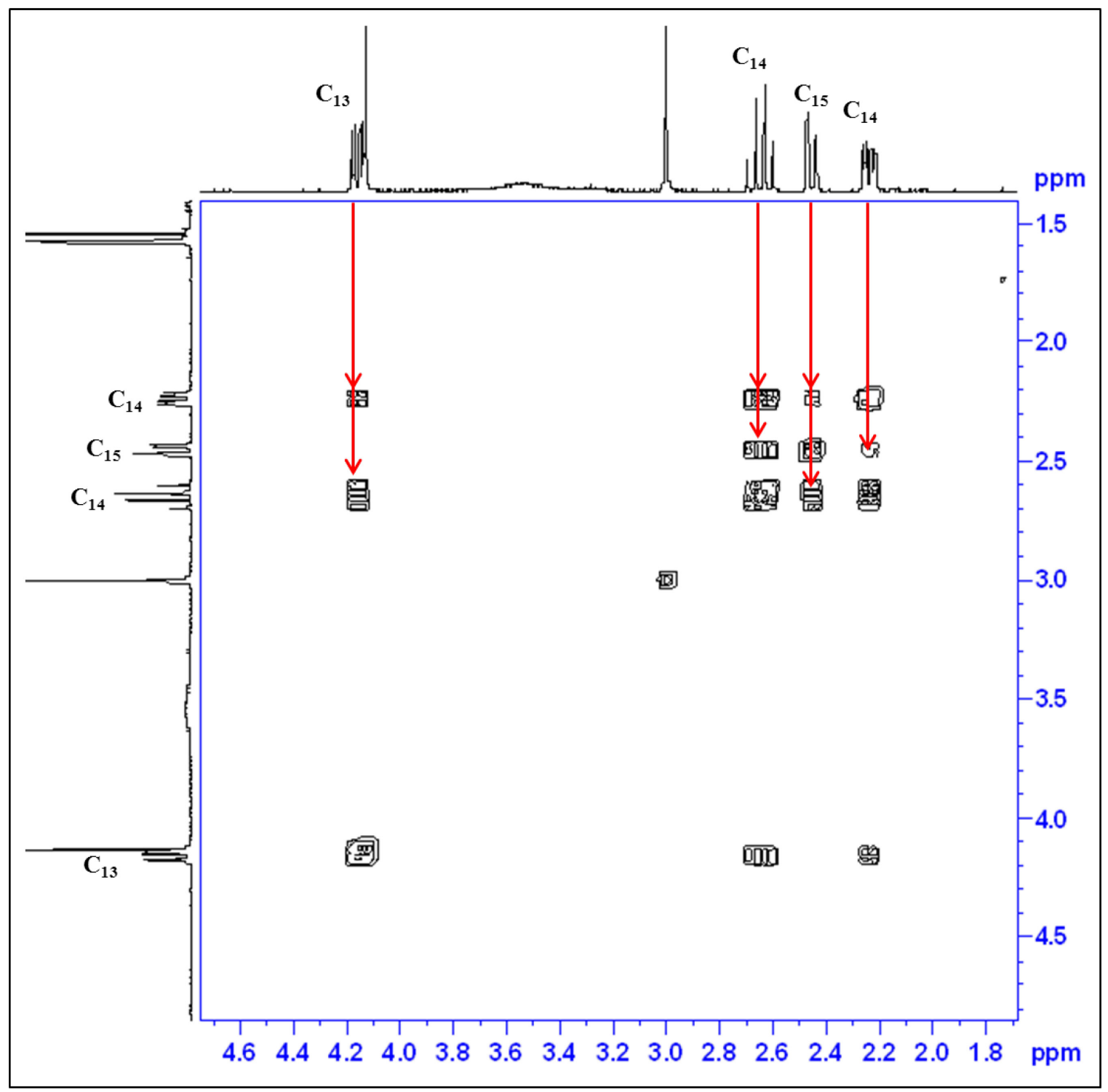

Figure 54: COSY of C13-C15 of 2

The aliphatic region of the NMR showed five singlets that integrated to 3 protons each, and a doublet that integrated to 1 proton (Figure 55). These chemical shifts were labeled as the five methyl groups and the hydroxyl group observed in many ambiguines including Ambiguine B Isonitrile. Each of the methyl groups were shown to correlate to a carbon chemical shift but the assignment of the methyl groups was only possible with the NOESY data. 


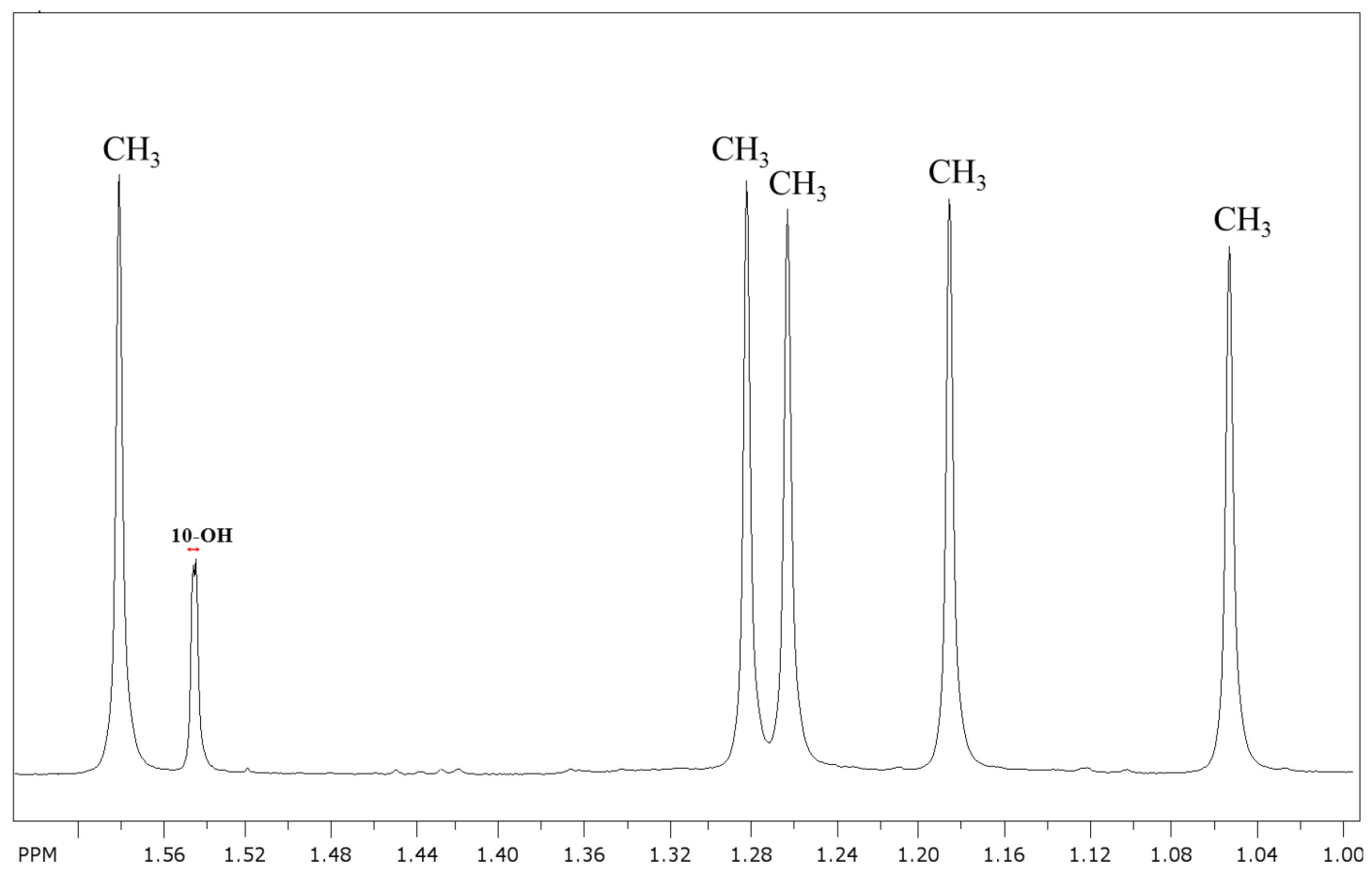

Figure 55: Aliphatic Region of the $1 \mathrm{H}$ NMR of 2

Given the large number of similarities observed in the ${ }^{1} \mathrm{H}$ NMR data of Ambiguine B Isonitrile and $\mathbf{2}$ it was determined that $\mathbf{2}$ had the same hydrogen skeleton as Ambiguine B Isonitrile. The comparison provides much of the assignment of the hydrogen bearing carbons, and agrees with the tetracyclic ambiguines discussed in Chapter 1. The tetracyclic ambiguine structure contains an isonitrile group on the C11 carbon in the axial position. The lack of the isonitrile group was previously determined by IR and ${ }^{13} \mathrm{C} \mathrm{NMR}$, and the assignment was made of a hydrogen on $\mathrm{C} 11$. Whether the hydrogen was axial or equatorial was not able to be determined from the ${ }^{1} \mathrm{H},{ }^{13} \mathrm{C}, \mathrm{COSY}$, DEPT, and HMQC NMR. The chemical shift of the proton was downfield which suggests a heteroatom or unsaturated functional group was attached to the same carbon. The identity of the functional group was not able to be determined with the NMR data so all of the carbons and protons were accounted for and the remaining atoms were utilized 
to propose a structure. The location of the chlorine was deduced to be on C13 as a result of the coupling with two hydrogens in a cyclohexane ring that were shown to correlate to the same carbon, C14. The assignment of the hydroxyl group was done on the basis of the structures of previously isolated ambiguine and hapalindoles which places the hydroxyl group on C10. The assignment and positions of the hydroxyl group, each of the terminal alkenes, and the five methyl groups were not able to be deduced fully without the NOESY data.

From the ${ }^{1} \mathrm{H}$ NMR, ${ }^{13} \mathrm{C}$ NMR, DEPT, COSY, and HMQC, the majority of the hydrogens were assigned along with the corresponding carbons through the HMQC analysis. The remaining hydrogens were assigned using the NOESY data, and numbered using the structure in Figure 56.

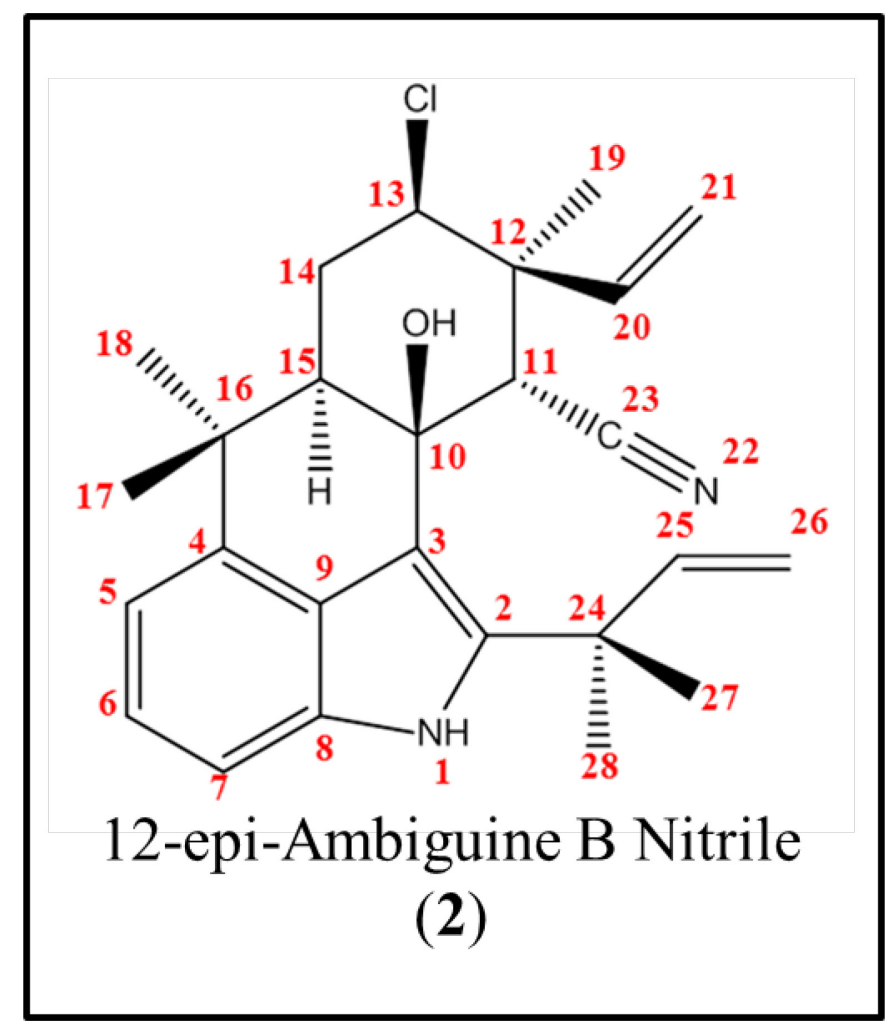

Figure 56: Structure and Numbering of 2 
The hydroxyl group was assigned to C10. In the NOESY NMR analysis, the doublet produced by the hydroxyl group was shown to correlate to two methyl groups, the doublet of doublets at $5.05 \delta$, and the singlet assigned as the hydrogen on C11. Since the hydrogen on $\mathrm{C} 15$ was determined to be axial the hydroxyl group would not spatially correlate to that hydrogen. From this analysis it was determined that the hydroxyl group was assigned correctly to $\mathrm{C} 10$ since this position would afford spatial correlation to at least two methyl groups, the hydrogen on $\mathrm{C} 11$, and either of the terminal alkenes depending on the stereochemistry.

The doublet of doublets at $5.05 \delta$ were shown to couple to the doublet of doublets at $6.78 \delta$ and $5.09 \delta$. The carbons bearing these hydrogens were shown to match closely to the chemical shifts of the $\mathrm{C} 20$ and $\mathrm{C} 21$ carbons. The positions of these carbons were investigated using the NOESY. The quartet at $2.65 \delta$, assigned as the C14 axial proton, was shown to produce a correlation with the doublet of doublets at $6.78 \delta$. With this correlation it was determined that the terminal alkene was attached to the cyclohexane ring. Given the structure of the cyclohexane ring the axial position of the $\mathrm{C} 14$ carbon positions out of the $\mathrm{x}, \mathrm{y}$ plane. With the observed correlation in the NOESY data the alkene normally observed on the $\mathrm{C} 12$ carbon in most of these indole alkaloids must also position out of the $\mathrm{x}, \mathrm{y}$ plane to produce the observed correlation. With the deduction of the position of the alkene the remaining methyl group also normally located on the $\mathrm{C} 12$ carbon in these indole alkaloids must be positioned towards the back of the plane. The configuration of the four carbons bonded to the $\mathrm{C} 12$ carbon includes the cyclohexane bonded carbons oriented in the plane, the methyl group positioned to the back of the plane and the terminal alkene positioned coming out of the plane. The observed 
orientation of the functional groups suggests a $\mathrm{C} 12$ epimer sometimes observed with the hapalindoles (Klein et al., 1995). The remaining terminal alkene was deduced to be the C25-C26 position normally observed in ambiguines. The assignment was made on the basis of the chemical shift of the carbons shown to correlate to carbons C25 and C26 in Ambiguine B Isonitrile. In order to confirm this assignment the methyl groups normally attached to $\mathrm{C} 24$ would have to correlate to at least one set of doublet of doublets produced by $\mathrm{C} 25$ or $\mathrm{C} 26$ hydrogens.

Assignment of the positions of the five methyl groups was necessary to propose the ambiguine structure. The singlet at $1.58 \delta$ was assigned as the $\mathrm{C} 19$ methyl group, on the basis of the correlations observed in the NOESY. The singlet was shown to correlate to the doublet of doublet produced by the $\mathrm{C} 13$ proton, the singlet assigned as the proton on $\mathrm{C} 11$, and the doublet of doublets assigned as $\mathrm{C} 21 \mathrm{E}$ proton. The correlation to the C13 proton suggests a methyl attached to the C12 carbon normally observed in ambiguines and hapalindoles. Which the observed correlations the singlet at $1.58 \delta$ was labeled as the methyl group C19.

The remaining four methyl groups were attached to two carbons, C16 and C24. The methyl groups attached to $\mathrm{C} 24$ should correlate to $\mathrm{C} 25$ or C26 hydrogens. The singlet at 1.05 was shown to correlate to the singlet assigned as $\mathrm{C} 11$, the $\mathrm{C} 10$ hydroxyl group, and the $\mathrm{C} 26 \mathrm{E}$ proton. The correlations suggest the methyl group was most likely attached to the $\mathrm{C} 24$ carbon. Given that the hydroxyl group was assigned as coming out of the $\mathrm{x}, \mathrm{y}$ plane as a result of the NOESY correlation to the $\mathrm{C} 11$ proton and the $\mathrm{C} 19$ methyl group, the orientation of the methyl group was determined to be coming out of the $\mathrm{x}, \mathrm{y}$ 
plane thereby assigning the methyl as C27. The carbon chemical shift of $29.45 \delta$ closely matched the shift produced by $\mathrm{C} 27$ of Ambiguine B Isonitrile.

The singlet at $1.18 \delta$ was determined to be a methyl group attached to the $\mathrm{C} 16$ carbon of one of the cyclohexane rings. The singlet was observed to correlate to the $14 \mathrm{ax}$ proton and the hydroxyl group on C10. The 14ax proton was oriented coming out of the plane thereby assigning the methyl as also coming out of the plane to allow spatial correlation. In Ambiguine B Isonitrile, the methyl group attached to C16, and oriented coming out of the plane, was labeled as the $\mathrm{C} 17$ methyl. From the similar observations the singlet at $1.18 \delta$ was assigned as the protons on C17. From the HMQC the singlet was shown to correlate to a chemical shift of $27.2 \delta$ which closely matched the chemical shift of $26.5 \delta$ reported for $\mathrm{C} 17$ in ambiguine B Isonitrile.

The remaining two methyl groups, that produced singlets at 1.26 and $1.28 \delta$, were shown to only differ by $0.2 \delta$ in the ${ }^{1} \mathrm{H}$ NMR. The NOESY was not able to resolve the correlations, and therefore different regions of the proposed structure were shown to correlate to the two methyl singlets. By evaluating the ambiguine structure it was determined that the two singlets most likely were the methyls C18 and C28. The singlet at $1.26 \delta$ was shown to correlate to the carbon chemical shift at $28.1 \delta$ which matches the 27.68 reported for $\mathrm{C} 28$ in Ambiguine B Isonitrile. The singlet at $1.28 \delta$ correlated to a carbon chemical shift at around $27.6 \delta$ in the HMQC. Unfortunately the C19 methyl group also correlated to a carbon chemical shift at around 27.68. From all of the NOESY and HMQC data, the singlets observed in the ${ }^{1} \mathrm{H}$ NMR could be assigned, however, the carbon assignments of $\mathrm{C} 19$ and $\mathrm{C} 18$ were not possible. Therefore, from the inability to resolve the carbon-hydrogen correlations, the $\mathrm{C} 19$ and $\mathrm{C} 18$ carbon assignments were only 
relative compared to the carbon chemical shifts to Ambiguine B Isonitrile From the NOESY data the 3 dimensional structure was deduced with the chiral centers 10R*, $11 \mathrm{~S}^{*}, 12 \mathrm{R}^{*}, 13 \mathrm{R}^{*}$, and $15 \mathrm{R}^{*}$.

Once all of the proton chemical shifts were assigned the quaternary carbons were left to assign. Using Ambiguine B Isonitrile as a template the remaining carbons were assigned by how similar the chemical shift of the carbons of $\mathbf{2}$ matched the assigned carbons in Ambiguine B Isonitrile. Majority of the carbons matched very closely to Ambiguine B Isonitrile with the exceptions of C11, C19, C20, C21, and C23. The discrepancy between the $\mathrm{C} 19$ and $\mathrm{C} 20-21$ was attributed to the orientation of the substituents on $\mathrm{C} 12$. The difference observed with $\mathrm{C} 11$ was attributed to the unidentified functional group attached to $\mathrm{C} 11$. Even with the difference in carbon chemical shifts, the ${ }^{1} \mathrm{H}$ NMR corroborates the assignment of all of the carbons except C23. Once all of the carbons were assigned one carbon chemical shift was left with a value of $120.1 \delta$. The unassigned carbon was determined to be quaternary from analyzing the DEPT experiment. Also the formula produced from the mass spectrometric analysis specified two nitrogens, and only one had been assigned to the structure in the indole moiety. With this information many possible functional groups were eliminated. Even though the IR did not observe any peaks at $2100-2200 \mathrm{~cm}^{-1}$, the functional group was determined to be a nitrile on the basis of the carbon chemical shift which falls into the range of a nitrile, $110-140 \delta$, the quaternary nature of the unassigned carbon, and the remaining unassigned nitrogen (Pavia et al., 2009). With the assignment of the C11 proton as equatorial and oriented coming out of the $\mathrm{x}, \mathrm{y}$ plane the nitrile group was then assigned as axial which would decrease any steric interactions with the isoprenyl group attached to $\mathrm{C} 2$. 


\subsection{Conclusion}

It was proposed from the data that $\mathbf{1}$ was 12-epi-Hapalindole $\mathrm{H}$ Isonitrile and $\mathbf{2}$ was 12-epi-Ambiguine B Nitrile. The main evidence to support the structure of 1 was the carbon chemical shifts which matched very closely to the previously reported NMR data for 12-epi-hapalindole $\mathrm{H}$ Isonitrile, and the IR detection of the isonitrile group (Klein et al., 1995). The nitrile group proposed in 2 was assigned using the remaining atoms not accounted for in the formula. However, the remaining parts of the structure closely matched the structure of Ambiguine B Isonitrile except for the stereochemistry of C12. In order to confirm the nitrile group, an IR would have to be done on $\mathbf{2}$ either in liquid phase or using a larger amount of solid. In Chapter 1 it was noted that many isonitrile containing compounds had pronounced bioactivity. From the toxicological evaluations of $\mathbf{1}$ and $\mathbf{2 , 1}$ was shown to be discerningly more bioactive than $\mathbf{2}$ which might be caused by the occurrence of an isonitrile group in $\mathbf{1}$ and the nitrile group in $\mathbf{2}$.

\subsection{Experimental Section}

\subsubsection{Purification}

Two sequential HPLC methods were used to purify indole alkaloids from the optimized methanol extracts of ECS using a Shimadzu Prominence UFLC system, with a PDA detector to separate the indole containing compounds from the active extract. The indole chromophore observed in the active fraction had a $\lambda \max$ at 220-225 and 278-282 nm. The chromatographic methods were developed to separate the observed peaks with a $\lambda \max$ at $222 \mathrm{~nm}$. Each method utilized a Luna Semi-Prep C18 column with $5 \mu \mathrm{m}$ particle size and dimensions of $250 \times 10 \mathrm{~mm}$ with a flowrate of $4.5 \mathrm{~mL} / \mathrm{min}$. The first HPLC method used an acetonitrile and water gradient. The gradient began at 50\% acetonitrile 
and ended at 99\% acetonitrile in 20 minutes, and an isocratic gradient at $99 \%$ acetonitrile was kept for an additional 20 minutes. The indoles of interest were collected at 15 to 20 minutes, this was determined after a smaller scale collection was done and tested against the zebrafish embryo model. The collected fraction at 15-20 minutes was determined to be bioactive and was further fractioned using a second method. The second method used a very slight gradient of methanol and water which started with $73.2 \%$ methanol and was increased to $73.5 \%$ over the span of 40 minutes. The percent of methanol was again increased to $75 \%$ for the following 12 minutes which afforded eight different peaks with indole-like chromophores. Six fractions containing either a single large peak or a group of small peaks were collected and tested against the zebrafish embryo assay. The fractions containing Peaks 7 and 8 were determined to be pure. Peak 7 and 8 produced two amorphous white solids each of which was then analyzed using the TSQ Quantum Access using a data dependent scan to determine purity.

\subsubsection{Ultraviolet and Infrared Analysis}

The UV data was obtained from the PDA detector of the HPLC during fractionation. The IR was performed on a Perkin Elmer Spectrum IR using solid aliquots of each compound.

\subsubsection{Mass Spectrometer Analysis}

High resolution mass was determined by LC-MS, using an OT Velos Orbitrap Mass Spectrometer at the Thermo Scientific Teaching Center, with a $0.1 \%$ formic acid in water and $0.1 \%$ formic acid in acetonitrile gradient using an Heated Electrospray Ionization (HESI) source. High resolution mass spectrometry was not performed on the purified individual compounds. However, high resolution analysis of the active indole fraction of the ECS produced a number of ions with $\mathrm{m} / \mathrm{z}$ of 305 . In order to propose a 
high resolution mass for $\mathbf{1}$, the relative retention times of the eight peaks discussed in Chapter 2 were used to select the ion with an $\mathrm{m} / \mathrm{z}$ value of 305.24534 , which was shown to elute after Peak 7. This same order of elution was observed in various other conditions using the HPLC and LCMS. Analysis of $\mathbf{1}$ on the TSQ using acetonitrile did not produce any molecular ion, however, the ions were able to be observed in a methanol and water gradient. The proposed mass was the further evaluated using IR and NMR experiments. The mass of 2 was determined by filtering ions with an $\mathrm{m} / \mathrm{z}$ value of 423 with a chlorine isotope $[\mathrm{M}+2]^{+}$from the OT Velos data. Only one mass was observed and was used to propose a formula. In the TSQ Quantum data, masses not produced by the compound were determined by comparison to a HPLC solvent blank to account for any impurities produced from the column or solvents. These masses were ignored in the final analysis.

\subsubsection{Nuclear Magnetic Resonance Spectroscopic Analysis}

NMR analyses were performed on a Bruker $400 \mathrm{MHz}$ NMR, and experiments included, ${ }^{1} \mathrm{H}$ NMR, ${ }^{13} \mathrm{C}$ NMR, DEPT 135, COSY, HMQC, and NOESY. The analyses were performed using $\mathrm{C}_{6} \mathrm{D}_{6}$ for both, MeOD for $\mathbf{2}$, and MeOD and a small amount of $\mathrm{D}_{2} \mathrm{O}$ for 1. Many previously identified indole alkaloids from Stigonemataceae were analyzed with the NMR using $d$-chloroform. However, a study by Moore et al., (1992) observed that the normally occurring isonitrile groups degrade in $d$-chloroform. The formation of the formamide from the isonitrile was observed to occur after three days in $d$-chloroform. This same reaction was utilized by Moore et al., (1988) to study the biosynthesis of the isonitrile group, where the formamide was formed from the isonitrile via acid hydrolysis. In order to avoid unwanted degradation the NMR analyses were not performed in $d$-chloroform. Chemical shift produced by each compounds were identified 
by comparison to a blank for each solvent and also with comparisons to previously identified indole alkaloids. The chemical shifts were calibrated using the methanol solvent shift in the MeOD samples and $0.05 \%$ TMS in the $\mathrm{C}_{6} \mathrm{D}_{6}$.

12-epi-Hapalindole H Isonitrile: amorphous white solid; IR $v_{\max } 3412$ and 2137 cm-1; UV (PDA) $\lambda \max 218,278,290 \mathrm{sh} n m ;{ }^{1} \mathrm{H}$ NMR (400MHz, $\left.\mathrm{C}_{6} \mathrm{D}_{6}\right) \delta$ (multiplicity, $\mathrm{J}$ in Hz, assignment; COSY); 6.75 (br s, 1-NH); 7.55 (dd, H-2); 7.05 (d, H-5, J = 7.3Hz; 6); 7.26 (dd, H-6, J = 7.3 and $8.0 \mathrm{~Hz} ; 5,7) ; 6.96$ (d, H-7, J = $8 \mathrm{~Hz} ; 6$ ); 3.09 (dd, H-10ax, J = 11 and 10.8 Hz; 11, 15); 3.21 (br d, H-11ax, J = 11 Hz; 10); 0.97 (m, H-13ax; 13eq, 14ax, 14 eq); 1.33 (m, H-13eq; 13ax, 14ax, overlap 14eq); 1.15 (m, H-14ax; 13ax, 13eq, 14eq); 1.33 (m, H-14 eq; 14ax, 13ax, overlap 13eq); 1.03 (m, H-15; 10, 13ax, 13eq, 14ax, 14eq); 0.96 (s, H3-17); 1.28 (s, H3-18); 1.10 (s, H3-19); 5.73 (dd, H-20, J = 17.4 and 10.8Hz; 21E, 21Z); 5.08 (dd, H21E, J = 17.4 and $0.8 \mathrm{~Hz} ; 20,21 \mathrm{Z}) ; 5.06$ (dd, H-21Z, 10.8 and 0.8 $\mathrm{Hz} ; 20,21 \mathrm{E}) ;{ }^{13} \mathrm{C}$ NMR $\left(400 \mathrm{MHz}, \mathrm{C}_{6} \mathrm{D}_{6}\right) \delta$ (position); 118.7 (C-2);113.4 (C-3); 140.8 (C4); 113.0 (C-5); 123.1 (C-6); 108.6 (C-7); 134.0 (C-8); 125.8 (C-9); 36.3 (C-10); 65.6 (C11); 37.3 (C-12); 36.0 (C-13); 20.6 (C-14); 49.6 (C-15); 40.6 (C-16); 24.7 (C-17); 24.9 (C-18); 17.0 (C-19); 145.9 (C-20); 113.6 (C-21); HESIMS m/z 278 ([M-HCN]+, 305 $([\mathrm{M}+\mathrm{H}]+), 609([2 \mathrm{M}+\mathrm{H}]+)$; HRHESIMS m/z $305.24534\left(\mathrm{C}_{21} \mathrm{H}_{24} \mathrm{~N}_{2}, \Delta+44 \mathrm{mmu}\right)$.

12-epi-Ambiguine B Nitrile: amorphous white solid; IR $v_{\max } 3378$ and $1616 \mathrm{~cm}$ 1; UV (PDA) $\lambda \max 222,279,290 \mathrm{sh} \mathrm{nm} ;{ }^{1} \mathrm{H}$ NMR $\left(400 \mathrm{MHz}, \mathrm{C}_{6} \mathrm{D}_{6}\right) \delta$ (multiplicity, J in Hz, assignment; COSY; NOESY); 8.04 (br s, 1-NH); 7.10 (dd, H-5, J = 0.5, 7.3Hz; 6; 18); 7.27 (dd, H-6, J = 7.3 and $8.0 \mathrm{~Hz} ; 5,7 ; 18) ; 6.96$ (dd, H-7, J = 0.5, $8 \mathrm{~Hz} ; 6$ ); 4.13 (s, H-11eq; na;10-OH, 19, 27, 28); 4.16 (dd, H-13ax, J = 12.6 and $4.3 \mathrm{~Hz} ; 14 \mathrm{ax}, 14$ eq; 19, 14ax, 14eq, 15); 2.65 (q, H-14ax, J = 12.6Hz; 13eq, 14eq, 15ax; 13, 17, 20); 2.25 (ddd, 
$\mathrm{H}-14$ eq, $\mathrm{J}=13,4.3,2.8 \mathrm{~Hz} ; 14 \mathrm{ax}, 13 \mathrm{ax}, 15 ; 18,13) ; 2.45$ (dd, H-15, J = 12.6 and $2 \mathrm{~Hz}$; 14ax, 14eq; 18, 13); 1.05 (s, H3-17; ; 10-OH, 14ax); 1.58 (s, H3-18; ; 5, 6, 14eq, 15); 1.18 (s, H3-19; ; 11, 13, 21E); 6.78 (dd, H-20, J = 17.6 and $11 \mathrm{~Hz} ; 21 \mathrm{E}, 21 \mathrm{Z} ; 14 \mathrm{ax}, 21 \mathrm{E}$, 21Z); 5.05 (dd, H21E, J = 17.6 and 0.5 Hz; 20, 21Z; 19, 20, 21Z); 4.69 (dd, H-21Z, 10.5 and $0.7 \mathrm{~Hz} ; 20,21 \mathrm{E} ; 20,21 \mathrm{E}) ; 6.05(\mathrm{dd}, \mathrm{H}-25, \mathrm{~J}=17.6$ and $10.5 \mathrm{~Hz} ; 26 \mathrm{E}, 26 \mathrm{Z} ; 26 \mathrm{E}$, 26Z); 4.69 (dd, H-26E, $\mathrm{J}=17.6$ and $0.7 \mathrm{~Hz} ; 25,26 \mathrm{Z}, 27,28) ; 5.05(\mathrm{dd}, \mathrm{H}-26 \mathrm{Z}, \mathrm{J}=11$ and $>0.5 \mathrm{~Hz} ; 25,26 \mathrm{E} ; 25,26 \mathrm{E}) ; 1.26$ (s, H3-27; ; 10-OH, 11, 26E); 1.28 (s, H3-28; ; 26E); 1.54 (d, 10-OH; J = 5.2 Hz; 20;11, 27, 17); ${ }^{13} \mathrm{C}$ NMR (400MHz, $\left.\mathrm{C}_{6} \mathrm{D}_{6}\right) \delta$ (position); 138.9 (C-2);112.2 (C-3); 140.3 (C-4); 115.0 (C-5); 123.3 (C-6); 108.1 (C-7); 132.3 (C-8); 125.7 (C-9); 73.3 (C-10); 53.8 (C-11); 44.3 (C-12); 66.8 (C-13); 29.9 (C-14); 51.4 (C-15); 37.0 (C-16); 26.6 (C-17); 27.0 (C-18); 27.1 (C-19); 142.1 (C-20); 113.9 (C-21); 119.5 (C-23); 38.9 (C-24); 147.4 (C-25); 112.7 (C-26); 28.8 (C-27); 27.5 (C-28); HESIMS m/z 421/423 (3:1 [M-H] $]^{-}$ion cluster); HESIMS m/z 423/425 $\left(3: 1[\mathrm{M}+\mathrm{H}]^{+}\right.$ion cluster); HRHESIMS $\mathrm{m} / \mathrm{z} 422.2128\left(\mathrm{C}_{26} \mathrm{H}_{31} \mathrm{OClN}_{2}, \Delta+0.9 \mathrm{mmu}\right)$. 


\section{REFERENCES}

1. Adekunle, O. K.; Acharya, R.; Singh, B., 2007. Toxicity of pure compounds isolated from Tagetes minuta oil to Meloidogyne incognita. Australian Plant Disease Notes. 2, 101-104.

2. Arillo, A.; Bavestrello, G.; Burlando, B.; Sara, M., 1993. Metabolic integration between symbiotic cyanobacteria and sponges: a possible mechanism Marine Biology. 117, 159-162.

3. Asthana, R.K.; Srivastava, A.; Singh, A. P.; Deepali; Singh, S.P.; Nath, G.; Srivastava, R.; Srivastava, B. S., 2006. Identification of an antimicrobial entity from the cyanobacterium Fischerella sp. isolated from bark of Azadirachta indica (Neem) tree. Journal of Applied Phycology. 18, 33-39.

4. Becher, P.G.; Keller, S.; Jung, G.; Sussmuth, R.D.; Juttner, F., 2007. Insecticidal activity of 12-epi-hapalindole J isonitrile. Phytochemistry. 68, 2493-2497.

5. Berry, J. P.; Gantar, M.; Gawley, R. E.; Wang, M.; Rein, K. S., 2004. Pharmacology and toxicology of pahayokolide A, a bioactive metabolite from a freshwater species of Lyngbya isolated from the Florida Everglades Comp. Biochem. Physiol. C Toxicol. Pharmacol. 139, 231-238.

6. Berry, J. P.; Gantar, M.; Gibbs, P.D.L.; Schmale, M.C., 2007. The zebrafish (Danio rerio) embryo as a model system for identification and characterization of developmental toxins from marine and freshwater microalgae. Comparative Biochemistry and Physiology, Part C. 145, 61-72.

7. Berry, J.; Perez, M.; Gantar, M.; Berry, G.; Noriega, F., 2008. Cyanobacterial Toxins as Allelochemicals with Potential Applications as Algaecides, Herbicides and Insecticides. Mar. Drugs. 6, 117-146.

8. Berry, J. P. Gibbs, P. D. L.; Schamle, M. C.; Saker, M. L., 2009. Toxicity of cylindrospermopsin, and other apparent metabolites from Cylindrospermopsis raciborskii and Aphanizomenon ovalisporum, to the zebrafish (Danio rerio) embryo. Toxicon. 53, 289-299. 
9. Bisby, F.A.; Roskov, T.R.; Orrell, T.M.; Nicolson, D.; Paglinawan, L.E.; Bailly, N.; Kirk, P.M.; Bourgoin, T.; Baillargeon, G., eds (2010). Species 2000 \& IT IS Catalogue of Life: 2010 Annual Checklist. Digital resource at http://www.catalogueoflife.org/annual-checklist/2010. Species 2000: Reading, UK.

10. Bold, H.C.; Wynne, M.J. "Introduction to the Algae Structure and Reproduction"; Prentice-Hall, Inc: New Jersey, 1987; pg 59-60.

11. Bonjouklian, R.; Moore, R. E.; Patterson, G. M. L., 1988. Acid-Catalyzed Reactions of Hapalindoles. J. Org. Chem. 53, 5866-5870.

12. Bonjouklian, R.; Spangle, L. A.; Moore, R. E., 1989. Facile Intramolecular Photoaddition and Oxidative Dimerization of Hapalindole E, a Naturally Occurring Isonitrile-Containing Indole. J. Org. Chem. 54, 719-721.

13. Bornemann, V.; Patterson, G.; Moore, R., 1988. Isonitrile Biosynthesis in the Cyanophyte Hapalosiphon fontinalis. J. Am. Chem. Soc. 110, 2339-2340.

14. Codd, G.A.; Bell, S.G.; Kaya, K.; Ward, C.J; Beattie, K.A.; Metcalf, J.S., 1999. Cyanobacterial toxins, exposure routes and human health. Eur. J. Phycol. 34, 405415.

15. Codd, G.A.; Morrison, L.F.; Metcalf, J.S., 2005. Cyanobacterial toxins: risk management for health protection. Toxicology and Applied Pharmacology. 203, 264-272.

16. Crawford, A. D.; Esguerra, C. V.; de Witte, P., 2008. Fishing for Drugs from Nature: Zebrafish as a Technology Platform for Natural Product Discovery. Planta Medica. 74, 624-632.

17. Doan, N.; Rickards, R.; Rothschild, J.; Smith, G., 2000. Allelopathic actions of the alkaloid 12-epi-hapalindole E isonitrile and calothrixin A from cyanobacteria of the genera Fischerella and Calothrix. Journal of Applied Phycology. 12, 409416. 
18. Doan, N. T.; Stewart, P. R.; Smith, G. D., 2001. Inhibition of bacterial RNA polymerase by the cyanobacterial metabolites 12-epi-hapalindole $\mathrm{E}$ isonitrile and calothrixin A. FEMS Microbiology Letters. 196, 135-139.

19. Etchegaray, A.; Rabello, E.; Dieckmann, R.; Moon, D. H.; Fiore, M. F.; von Dohren, H.; Tsai, S. M.; Neilan, B. A., 2004. Algicide production by the filamentous cyanobacterium Fischerella sp. CENA 19. Journal of Applied Phycology. 16, 237-243.

20. Evans, J. R.; Napier, E. J.; Yates, P., 1976. Isolation of a New Antibiotic from a Species of Pseudomonas. The Journal of Antibiotics. 29, 850-852.

21. Falch, B.S.; Konig, G.M.; Wright, A.D.; Sticher, O., 1993. Ambigol A and B: New Biologically Active Polychlorinated Aromatic Compounds from the Terrestrial Blue-Green Alga Fischerella ambigua. J. Org. Chem. 58, 6570-6575.

22. Foster, M. P.; Concepcion, G. P.; Caraan, G. B.; Ireland, C. M., 1992. Bistratamides $\mathrm{C}$ and D. Two New Oxazole-Containing Cyclic Hexapeptides Isolated from a Philippine Lissoclinum bistratum Ascidian. J. Org. Chem. 57, 6671-6675.

23. Fiore, M.F.; Genuario, D.B.; Pamplona da Silva, C.S.; Shishido, T.K.; Moraes, L.A.B.; Neto, R.C.; Silva-Stenico, M.E., 2009. Microcystin production by a freshwater spring cyanobacterium of the genus Fischerella. Toxicon. 83, 754-761.

24. Gantar, M.; Berry, J.; Thomas, S.; Wang, M.; Perez, R.; Rein, K., 2008. Allelopathic activity among Cyanobacteria and microalgae isolated from Florida freshwater habitats. FEMS Microbiol. Ecol. 64, 55-64.

25. Gross, E. M., 2003. Allelopathy of Aquatic Autotrophs. Critical Reviews in Plant Science. 22, 313-339.

26. Gugger, M.F.; Hofmann, L., 2004. Polyphyly of true branching cyanobacteria (Stigonematales). International Journal of Systematic and Evolutionary Microbiology. 54, 349-357. 
27. Hagmann, L.; Juttner, F., 1996. Fischerellin A, a Novel Photosystem-II-inhibiting Allelochemical of the Cyanobacterium Fischerella muscicola with Antifungal and Herbicidal Activity. Tetrahedron Letters. 37, 6539-6542.

28. Hill, A. J.; Teraoka, H.; Heideman, W.; Peterson, R. E., 2005. Zebrafish as a Model Vertebrate for Investigating Chemical Toxicity. Toxicological Sciences. 86, 6-19.

29. Huber, U.; Moore, R.E.; Patterson, G.M.L., 1998. Isolation of a NitrileContaining Indole Alkaloid from the Terrestrial Blue-Green Alga Hapalosiphon delicatulus. J. Nat. Prod. 61, 1304-1306.

30. Ikawa, M.; Sasner, J. J.; Haney, J. F., 2001. Activity of cyanobacterial and algal odor compounds found in lake waters on green alga Chlorella pyrenoidosa growth. Hydrobiologia. 443, 19-22.

31. Jimenez, J. I.; Huber, U.; Moore, R. E.; Patterson, G. M. L., 1999. Oxidized Welwitindolinones from Terrestrial Fischerella sp. J. Nat. Prod. 62, 569-572.

32. Klein, D.; Daloze, D.; Braekman, J.C., 1995. New Hapalindoles from the Cyanophyte Hapalosiphon laingii. J. Nat. Prod. 58, 1781-1785.

33. Knowles, C. J., 1976. Microorganisms and Cyanide. Bacteriological Reviews. 40, 652-680.

34. Leao, P. N.; Pereira, A. R.; Liu, W.-T.; Ng, J.; Pevzner, P. A.; Dorrestein, P. C.; Konig, G. M.; Vasconcelos, V. M.; Gerwick, W. H., 2010. Synergistic allelochemicals from a freshwater cyanobacterium. PNAS. 107, 11183-11188.

35. LeFebvre, K. A.; Trainer, V. L.; Scholz, N. L., 2004. Morphological abnormalities and sensorimotor deficits in larval fish exposed to dissolved saxitoxin. Toxicon. 66, 159-170.

36. Leflaive, J.; Ten-Hage, L., 2007. Algal and cyanobacterial secondary metabolites in freshwaters: a comparison of allelopathic compounds and toxins. Freshwater Biology. 52, 199-214. 
37. Mo, S.; Krunic, A.; Chlipala, G.; Orjala, J., 2009. Antimicrobial Ambiguine Isonitriles from the Cyanobacterium Fischerella ambigua. J. Nat. Prod. 72, 894899.

38. Mo, S.; Krunic, A.; Santarsiero, B.D.; Franzblau, S.G.; Orjala, J., 2010. Hapalindole-related alkaloids from the cultured cyanobacterium Fischerella ambigua. Phytochemistry. 71, 2116-2123.

39. Moore, R.E.; Cheuk, C.; Patterson, G.M.L., 1984. Hapalindoles: New Alkaloids from the Blue-Green Alga Hapalosiphon fontinalis. J. Am. Chem. Soc. 106, 64566457.

40. Moore, R.E.; Cheuk, C.; Yang, X.Q.G.; Patterson, G.M.L., 1987. Hapalindoles, Antibacterial and Antimycotic Alkaloids from the Cyanophyte Hapalosiphon fontinalis. J. Org. Chem. 52, 1036-1043.

41. Moore, R.E.; Yang, X.Q.G.; Patterson, G.M.L., 1987. Fontonamide and Anhydrohapaloxindole A, Two New Alkaloids from the Blue-Green Alga Hapalosiphon fontinalis. J. Org. Chem. 52, 3773-3777.

42. Moore, R.E.; Yang, X.Q.G.; Patterson, G.M.L.; Bonjouklian, R.; Smitka, T. A., 1989. Hapalonamides and Other Oxidized Hapalindoles from Hapalosiphon fontinalis. Phytochemistry. 28, 1565-1567.

43. Oberemm, A.; Becker, J.; Codd, G.A.; Steinberg, C., 1999. Effects of Cyanobacterial Toxins and Aqueous Crude Extracts of Cyanobacteria on the Development of Fish and Amphibians. Cyanotoxins and Fish Development. 7788.

44. Ogino, J.; Moore, R.E.; Patterson, G.M.L.; Smith, C.D., 1996. Dendroamides, New Cyclic Hexapeptides from a Blue-Green Alga. Multidrug-Resistance Reversing Activity of Dendroamide A. J. Nat. Prod. 59, 581-586. 
45. Papendorf, O.; Konig, G. M.; Wright, A. D.; Chorus, I.; Oberemm, A., 1997. Mueggelone, a Novel Inhibitor of Fish Development from the Fresh Water Cyanobacterium Aphanizomenon flos-aquae. J. Nat. Prod. 60, 1298-1300.

46. Papke, U.; Gross, E.M. Francke, W., 1997. Isolation, Identification and Determination of the Absolute Configuration of Fischerellin B. A New Algicide from the Freshwater Cyanobacterium Fischerella muscicola (Thuret). Tetrahedron Letters. 38, 379-382.

47. Park, Aeri,; Moore, R.E.; Patterson, G.M.L., 1992. Fischerindole L, a New Isonitrile from the Terrestrial Blue-Green Alga Fischerella muscicola. Tetrahedron Letters. 33, 3257-3260.

48. Pavia, D. L.; Lampman, G. M.; Kriz, G. S. Introduction to Spectroscopy $4^{\text {th }}$ ed. 2009.

49. Philippis, R. D.; Vincenzini, M., 1998. Exocellular polysaccharides from cyanobacteria and their possible applications. FEMS Microbiology Reviews. 22, $151-175$.

50. Pistorius, E. K.; Jetschmann, K.; Voss, H.; Vennesland, B., 1979. The dark respiration of Anacystis nidulans: Production of $\mathrm{HCN}$ from histidine and oxidation of basic aminoacids. Biochim. Biophys. Acta. 585, 630-642. (b)

51. Pistorius, E. K.; Voss, H., 1980. Some properties of a basic L-amino-acid oxidase from Anacystis. Biochim. Biophys. Acta. 611, 227-240.

52. Prinsep, M. R.; Moore. R.E.; Levine, I.A.; Patterson, G.M.L., 1992. Westiellamide, a Bistratamide-Related Cyclic Peptide from the Blue-Green Alga Westiellopsis Prolifica. J. Nat. Prod. 55, 140-142.

53. Prinsep, M. R.; Caplan, F. R.; Moore, R. E.; Patterson, G. M. L.; Honkanen, R. E.; Boynton, A. L., 1992. Microcystin LA from a blue-green alga belonging to stigonematales. Phytochemistry. 31, 1247-1248. 
54. Purdie, E. L.; Samsudin, S.; Eddy, F. B.; Codd, G. A., 2009. Effects of the cyanobacterial neurotoxin $\beta$-N-methylamino-L-alanine on the early-life stage development of zebrafish (Danio rerio). Aquatic Toxicology. 95, 279-284.

55. Rao, D.R.; Thangavel, C.; Kabilan, L.; Suguna, S.; Mani, T.R.; Shanmugasundaram, S., 1999. Larvicidal properties of the cyanobacterium Westiellopsis sp. (blue-green algae) against mosquito vectors. Transactions of the Royal Society of Tropical Medicine and Hygiene. 93, 232.

56. Raveh, A.; Carmeli, S., 2007. Antimicrobial Ambiguines from the Cyanobacterium Fischerella sp. Collected in Israel. J. Nat. Prod. 70, 196-201.

57. Raven, P. H.; Johnson, G. B. Biology. $4^{\text {th }}$ ed. 1990. pgs. 270-280.

58. Richter, J.M.; Ishihara, Y.; Masuda, T.; Whitefield, B.W.; Llamas, T.; Pohjakallio, A.; Baran, P.S., 2008. Enantiospecific Total Synthesis of the Hapalindoles, Fischerindoles, and Welwitindolinones via a Redox Economic Approach. J. Am. Chem. Soc. 130, 17938-17954.

59. Schwartz, R. E.; Hirsch, C. F.; Springer, J. P.; Pettibone, D. J.; Zink, D. L., 1987. Unusual Cyclopropane-Containing Hapalindolinones from a Cultured Cyanobacterium. J. Org. Chem. 52, 3706-3708.

60. Schwartz, R.E.; Hirsch, C.F.; Sesin, D.F.; Flor, J.E.; Chartrain, M.; Fromtling, R.E.; Harris, G.H.; Salvatore, M.J.; Liesch, J.M.; Yudin, K., 1990. Pharmaceuticals from cultured algae. Journal of Industrial Microbiology. 5, 113124.

61. Simpson, J. S.; Garson, M., 1998. Thiocyanate Biosynthesis in the Tropical Marine Sponge Axinyssa n.sp. Tetrahedron Lett. 39, 5819-5822.

62. Simpson, J. S.; Garson, M., 1999. Advanced Precursors in Marine Biosynthetic Study: The Biosynthesis of Diisocyanoadociane in Amphimedon terpenensis. Tetrahedron Lett. 40, 3909. 
63. Simpson, J. S.; Garson, M., 2004. Marine isocyanides and related natural products - structure, biosynthesis and ecology. Nat. Prod. Rep. 21, 164-179.

64. Sivonen, K., 1996. Cyanobacterial toxins and toxin production. Phycologia. 35, 12-24.

65. Smitka, T.; Bonjouklian, R.; Doolin, L.; Jones, N.; Deeter, J.; Yoshida, W.; Prinsep, M.; Moore, R.; Patterson, G., 1992. Ambiguine Isonitriles, Fungicidal Hapalindole-Type Alkaloids from Three Genera of Blue-Green Algae Belonging to the Stigonemataceae. J. Org. Chem. 57, 857-861.

66. Stratmann, K.; Burgoyne, D.L.; Moore, R.E.; Patterson, G.M.L., 1994. Hapalosin, a Cyanobacterial Cyclic Depsipeptide with Multidrug-Resistance Reversing Activity. J. Org. Chem. 59, 7219-7226.

67. Stratmann, K.; Moore, R.; Bonjouklian, R.; Deeter, J.; Patterson, G.; Shaffer, S.; Smith, C.; Smitka, T., 1994. Welwitindolinones, Unusual Alkaloids from the Blue-Green Algae Hapalosiphon welwitschii and Westiella intricata. Relationship to Fischerindoles and Hapalindoles. J. Am. Chem. Soc. 116, 9935-9942.

68. Tan, G. T.; Pezzuto, J. M.; Kinghorn, A. D.; Hughes, S. H., 1991. Evaluation of Natural Products as Inhibitors of Human Immunodeficiency Virus Type 1 (HIV1) Reverse Transcriptase. J. Nat. Prod. 54, 143-154.

69. Tiedeken, J.A.; Ramsdell J. S.; Ramsdell, A. F., 2005. Developmental toxicity of domoic acid in zebrafish (Danio rerio). Neurotoxicol. Teratol. 27, 833-843.

70. Valente, S. A. D. S.; Valente, V. D. C.; Neves Pinto, A. Y. D.; Cesar, M. D. J. B.; Santos, M. P. D.; Miranda, C. O. S.; Cuervo, P.; Fernandes, O., 2009. Analysis of an acute Chagas disease outbreak in the Brazilian Amazon: human cases, triatomines, reservoir mammals and parasites. Transactions of the Royal Society of Tropical Medicine and Hygiene, 103, 291-297.

71. Van Wagoner, R.M.; Drummond, A.K.; Wright, J.L.C., 2007. Biogenetic Diversity of Cyanobacterial Metabolites. Advances in Applied Microbiology. 61, 89-182. 
72. Wang, P.-J.; Chien, M.-S.; Wu, F.-J.; Chou, H.-N.; Lee, S.-J., 2005. Inhibition of embryonic development by microcystin-LR in zebrafish, (Danio Rerio). Toxicon. 45, 303-308.

73. Wright, A. D.; Papendorf, O.; Konig, G. M., 2005. Ambigol C and 2,4Dichlorobenzoic Acid, Natural Products Produced by the Terrestrial Cyanobacterium Fischerella ambigua. J. of Nat. Prod. 68, 459-461.

74. Wright, A.D.; Papendorf, O.; Konig, G.M.; Oberemm, A., 2006. Effects of cyanobacterium Fischerella ambigua isolates and cell free culture media on zebrafish (Danio rerio) embryo development Chemosphere. 65, 604-608.

75. Zhang, $X$ and Smith, C.D., 1996. Microtubule effects of welwistatin, a cyanobacterial indolinone that circumvents multiple drug resistance. Molecular Pharmacology. 49, 288-294. 